\title{
HFIR Cold Source Upgrade Options
}

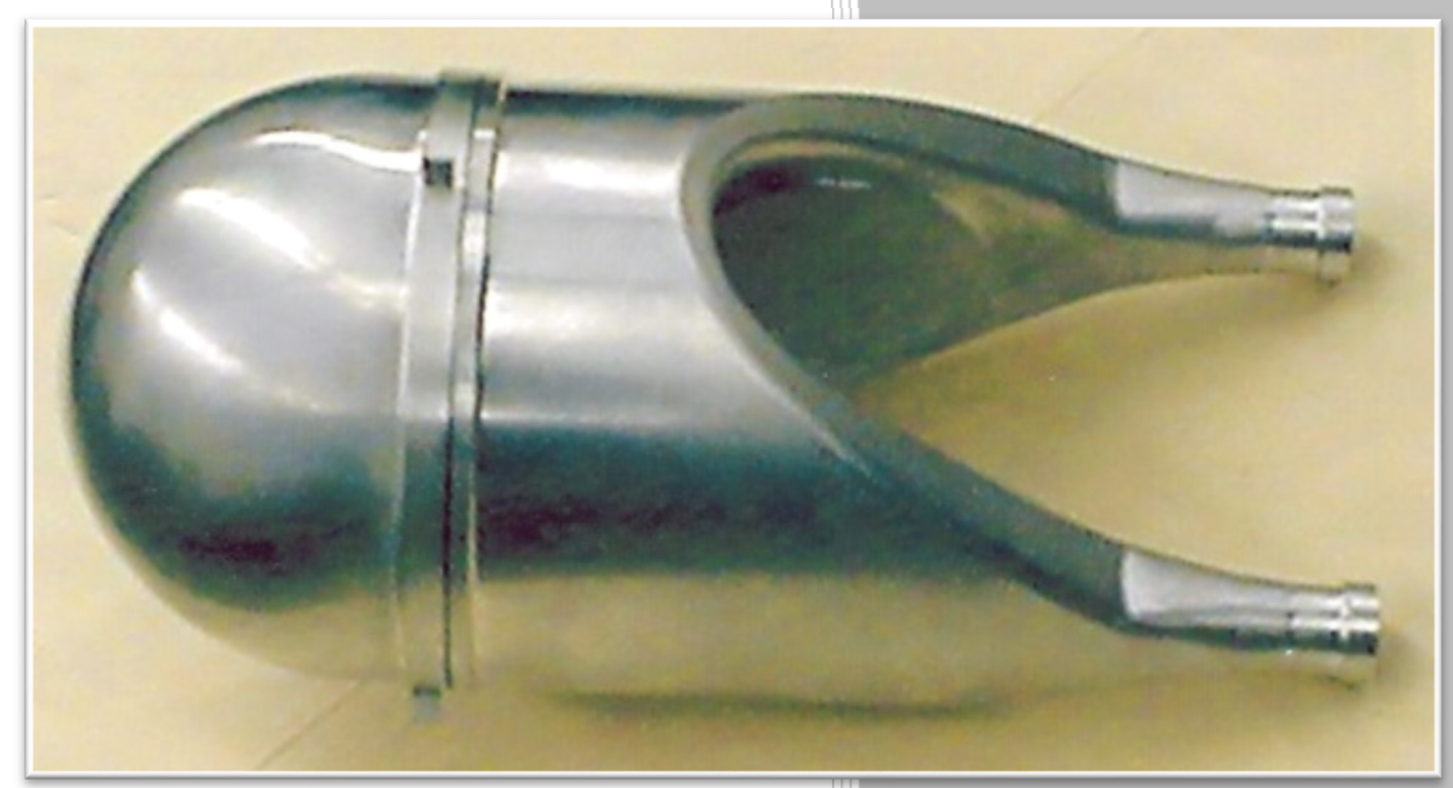

Approved for public release. Distribution is unlimited.

Igor Remec

Franz X. Gallmeier 


\title{
DOCUMENT AVAILABILITY
}

Reports produced after January 1, 1996, are generally available free via US Department of Energy (DOE) SciTech Connect.

Website http://www.osti.gov/scitech/

Reports produced before January 1, 1996, may be purchased by members of the public from the following source:

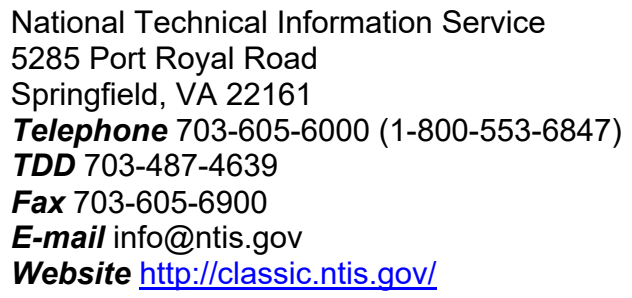

Reports are available to DOE employees, DOE contractors, Energy Technology Data Exchange representatives, and International Nuclear Information System representatives from the following source:

Office of Scientific and Technical Information

PO Box 62

Oak Ridge, TN 37831

Telephone 865-576-8401

Fax 865-576-5728

E-mail reports@osti.gov

Website http://www.osti.gov/contact.html

\begin{abstract}
This report was prepared as an account of work sponsored by an agency of the United States Government. Neither the United States Government nor any agency thereof, nor any of their employees, makes any warranty, express or implied, or assumes any legal liability or responsibility for the accuracy, completeness, or usefulness of any information, apparatus, product, or process disclosed, or represents that its use would not infringe privately owned rights. Reference herein to any specific commercial product, process, or service by trade name, trademark, manufacturer, or otherwise, does not necessarily constitute or imply its endorsement, recommendation, or favoring by the United States Government or any agency thereof. The views and opinions of authors expressed herein do not necessarily state or reflect those of the United States Government or any agency thereof.
\end{abstract}




\title{
HFIR COLD SOURCE UPGRADE OPTIONS
}

\author{
Igor Remec \\ Franz X. Gallmeier
}

Date Published: April 2018

\author{
Prepared by \\ OAK RIDGE NATIONAL LABORATORY \\ Oak Ridge, TN 37831-6283 \\ managed by \\ UT-BATTELLE, LLC \\ for the \\ US DEPARTMENT OF ENERGY \\ under contract DE-AC05-00OR22725
}




\section{CONTENTS}

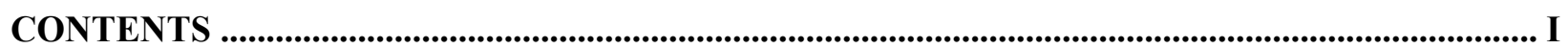

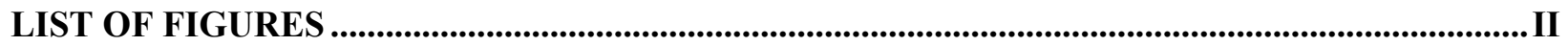

LIST OF TABLES ................................................................................................................................................

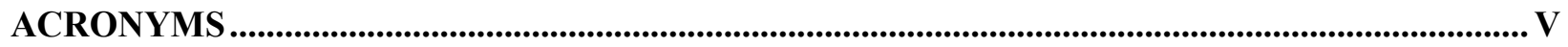

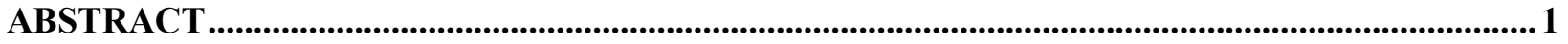

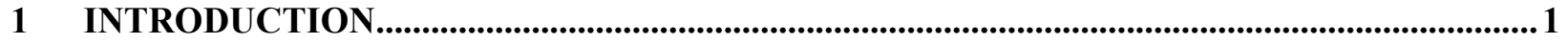

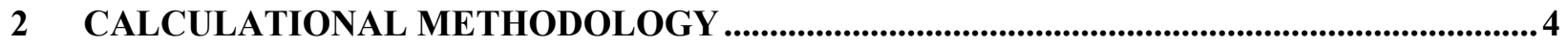

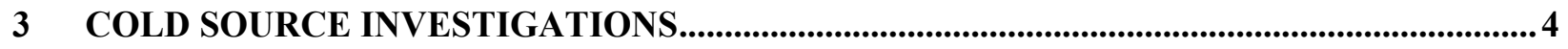

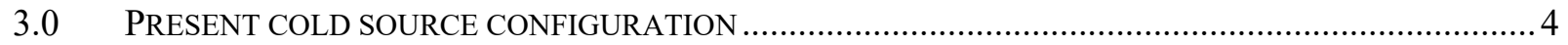

3.1 COLD SOURCE EXTENDED OUTWARD INTO HB-4 AT PRESENT CS RADIUS ................................ 10

3.2 COLD SOURCE EXTENDED INWARD INTO HB-4 AT PRESENT CS RADIUS ….............................. 11

3.3 COLD SOURCE EXTENDED INWARD INTO HB-4 AND INCREASED IN RADIUS ............................ 13

3.4 HB-1 AND HB-4 CONNECTED, COLD SOURCE CENTERED, AND LENGTH EXTENDED .................. 17

3.5 HB-1 AND HB-4 CONNECTED, COLD SOURCE SHAPE MODIFIED, AND LENGTH EXTENDED ........ 21

3.6 COMPARISON OF DETAILED CS BRIGHTNESS VERSUS ENERGY VARIATIONS ..........................28

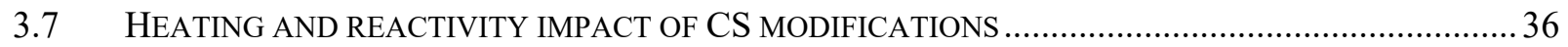

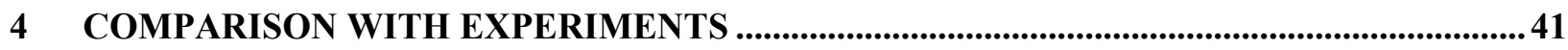

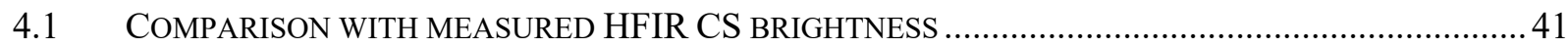

4.2 COMPARISON WITH MEASURED EFFECT OF PARA-HYDROGEN FRACTION .................................. 43

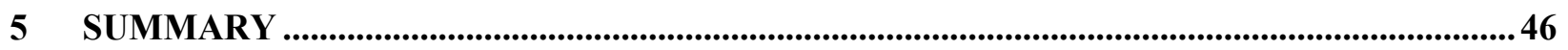

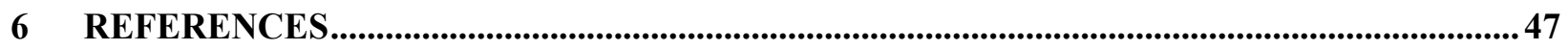




\section{LIST OF FIGURES}

Figure 1.0.1. Comparison of scattering cross section of ortho and para hydrogen and deuterium............. 2

Figure 3.0.1. Sketch of the present configuration of the HFIR cold source.............................................. 5

Figure 3.0.2. Horizontal section through the MCNP model of the HFIR reactor (top); detail of the region around the tip of the HB-1 and HB-4, with the cold source (bottom). ............................... 6

Figure 3.0.3. Brightness of the exiting CS, averaged over the viewed area $\left(68.63 \mathrm{~cm}^{2}\right.$, the full CS)

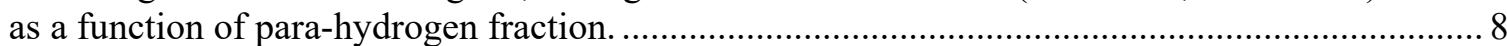

Figure 3.0.4. Brightness versus neutron energy (full energy range), for the existing CS configuration, actual location, viewed area $68.63 \mathrm{~cm}^{2}$ (the full CS), and two parahydrogen fractions.

Figure 3.0.5. Brightness versus neutron energy (below $1 \mathrm{eV}$ ), for the existing CS configuration, actual location, viewed area $68.63 \mathrm{~cm}^{2}$ (the full CS), and two para-hydrogen fractions.

Figure 3.1.1. Cold source configurations of existing design extending in length in the outward direction.

Figure 3.1.2. Brightness versus CS length, CS is extended outwards into the HB-4........................... 11

Figure 3.2.1. Cold source configurations of existing design extending in length in the inward direction.

Figure 3.2.2. Brightness versus CS length, CS is extended towards HB-1 and HB-1 is retracted by the same length.

Figure 3.3.1. Cold source configurations of existing design but beamtube and CS radii enlarged by $1 \mathrm{~cm}$ and extending in length in the inward direction.

Figure 3.3.2. Brightness versus CS length with CS increased in radius by $\sim 1 \mathrm{~cm}$ (viewed area is $104.23 \mathrm{~cm}^{2}$ ) and extended towards HB-1.

Figure 3.3.3. Brightness versus CS length with deuterium filled CS increased in radius by $\sim 1 \mathrm{~cm}$ (viewed area is $104.23 \mathrm{~cm}^{2}$ ) and extended towards the HB-1.

Figure 3.3.4. Brightness versus neutron energy (full energy range) for the reference CS, and extended CS (length $17 \mathrm{~cm}$ ) with increased radius, filled with hydrogen and deuterium.

Figure 3.3.5. Brightness versus neutron energy (for energies below $1 \mathrm{eV}$ ), for the reference CS, and extended CS (length $17 \mathrm{~cm}$ ) with increased radius, filled with hydrogen and deuterium.

Figure 3.4.1. Cold source configurations of existing design but placed in a through tube (connected HB-1 and HB-4 tube) and centered with regard to reactor core.

Figure 3.4.2. CS brightness for through tube arrangement, in the $0.13-5.1 \mathrm{meV}$ range $(25.1-4.0$

$\AA$ ), as a function of CS length and para-hydrogen fraction.

Figure 3.4.3. Brightness of the CS in through tube arrangement, as viewed from the HB-4 side, in the $0.13-5.1 \mathrm{meV}$ range $(25.1-4.0 \AA)$, as a function of CS length for various parahydrogen fractions.

Figure 3.4.4. Brightness of the CS in through tube arrangement, viewed from the HB-4 side versus CS length for $99 \%$ para-hydrogen fraction.

Figure 3.4.5. Brightness of the CS in through tube arrangement, viewed from the HB-1 side versus CS length for the $99 \%$ para-hydrogen fraction.

Figure 3.5.1. Examples of the CS configurations modified in shape.

Figure 3.5.2. Brightness of the shape modified CS viewed from HB-4 side, in the 0.13-5.1 meV range (25.1 - 4.0 $\AA$ ), as a function of para-hydrogen fraction and CS length (L) in the beam tube direction.

Figure 3.5.3. Brightness of the shape modified CS viewed from HB-4 side, in the $0.13-5.1 \mathrm{meV}$ range (25.1 - 4.0 $\AA$ ), as a function of CS length (L) for various CS thicknesses (T); parahydrogen fraction is $99 \%$. 
Figure 3.5.4. Brightness of the shape modified CS viewed from HB-1 side, in the 0.13-5.1 meV range (25.1 - 4.0 $\AA$ ), as a function of CS length (L) for various CS thicknesses (T); parahydrogen fraction is $99 \%$.

Figure 3.5.5. Brightness of the shape modified CS viewed from HB-4 side, as a function of CS length (L) in the beamline direction, for CS thickness (T) $8 \mathrm{~cm}$; for selected energy ranges; para-hydrogen fraction is $99 \%$.

Figure 3.5.6. Brightness of the shape modified CS viewed from HB-1 side, as a function of CS length (L) in the beamline direction, for CS thickness (T) $8 \mathrm{~cm}$; for selected energy ranges; para-hydrogen fraction is $99 \%$.

Figure 3.6.1. Cold source brightness versus energy, as viewed from the HB-4 side, for the reference $\mathrm{CS}$ and the five configurations shown in Table 3.6.1.

Figure 3.6.2. Cold source brightness versus energy (for energies below $1 \mathrm{eV}$ ), as viewed from the HB-4 side, for the reference CS and the five selected configurations.

Figure 3.6.3. Cold source brightness versus energy, relative to the reference cold source, as viewed from the HB-4 side, for the five selected configurations.

Figure 3.6.4. Cold source brightness versus energy, relative to the reference cold source, as viewed from the HB-4 side, for the five selected configurations, for the energies below $1 \mathrm{eV}$

Figure 3.6.5. Cold source brightness versus energy, for the reference CS and the two CS configurations with the through tube that allows viewing of the CS from HB-4 and HB-1 side.

Figure 3.6.6. Cold source brightness versus energy, for energies below $1 \mathrm{eV}$, for the reference CS and the two CS configurations with the through tube that allows viewing of the CS from HB-4 and HB-1 side.

Figure 3.6.7. Cold source brightness versus energy, relative to the reference cold source, for the two CS configurations with the through tube that allow viewing of the CS from HB-4 and HB-1 side.

Figure 3.6.8. Cold source brightness versus energy, relative to the reference cold source, for the two CS configurations with the through tube that allow viewing of the CS from HB-4 and HB-1 side, for the energies below $1 \mathrm{eV}$. ....

Figure 3.6.9. Brightness of the HB-1 side for the reference CS configuration and for the three CS configurations that keep the HB-4 and HB-1 separated.

Figure 3.6.10. Brightness of the HB-1 side relative to the HB-1 side brightness of the reference CS configuration, for the three CS configurations that keep the HB-4 and HB-1 separated.

Figure 4.1.1 Measured and calculated CS brightness versus energy.

Figure 4.1.2 Comparison of the measured and calculated CS brightness versus energy curves................ 42

Figure 4.2.1 Experimental apparatus used by Ooi and co-workers.

Figure 4.2.2 Neutron energy spectra and intensity ratios for the coupled moderator (left) and for the decoupled moderator (right). Intensity ratios are normalized by para-hydrogen $35 \%$ data......

Figure 4.2.3 Measured (at the right side) and simulated moderator brightness relative to the brightness of the moderator filled with $35 \%$ para-hydrogen. 


\section{LIST OF TABLES}

Table 3.0.1. Neutron scattering instruments on HFIR cold source and corresponding neutron energies and wavelengths of interest.

Table 3.0.2. CS brightness for the existing CS (present CS size and location), viewed area 68.63 $\mathrm{cm}^{2}$ (the full CS), and two para-hydrogen fractions, in selected energy ranges. ............................. 8

Table 3.5.1. Comparison of the CS brightness for the CS with inclined surfaces (Configuration A), and surfaces perpendicular to the beamline direction (Configuration B).

Table 3.5.2. CS brightness for the CS thickness $19 \mathrm{~cm}, 99 \%$ para-hydrogen fraction and different inclination angles Theta. Values are given relative to the value for THETA $=0$.

Table 3.6.1. Comparison of brightness and heating for selected cold source configurations. All values are given relative to the reference CS brightness and heating. ....................................... 28

Table 3.7.1. Heating of CS of existing design with CS length extended outward................................... 36

Table 3.7.2. Heating of existing CS with CS length extended inward................................................. 37

Table 3.7.3. Heating of CS with increased radius and with CS length extended inward........................... 38

Table 3.7.4. Heating of existing CS designs of various lengths placed in through tube* and centered around reactor core.

Table 3.7.5. Heating of modified CS designs of various lengths placed in through tube and centered around reactor core. 


\section{ACRONYMS}

$\begin{array}{ll}\text { CS } & \text { Cold Source } \\ \text { EOL } & \text { End of Life } \\ \text { ESS } & \text { European Spallation Source } \\ \text { HB } & \text { Horizontal Beamtube } \\ \text { HFIR } & \text { High Flux Isotope Reactor } \\ \text { JPARC } & \text { Japanese Proton Accelerator Research Complex } \\ \text { NASA } & \text { National Aeronautics and Space Administration } \\ \text { ORNL } & \text { Oak Ridge National Laboratory } \\ \text { PPU } & \text { Proton Power Upgrade project } \\ \text { SNS } & \text { Spallation Neutron Source } \\ \text { STS } & \text { Second Target Station project } \\ \text { VXF } & \text { Vertical Irradiation Facility }\end{array}$




\begin{abstract}
Several cold source configurations that provide $40 \%$ to $90 \%$ higher brightness than the reference cold source in broad ranges of wavelengths longer than $\sim 2 \AA$ were identified. These CS configurations use hydrogen volumes 2.5 to 4 times larger than the reference source, require a nearly pure para-hydrogen for operation (99\% para-hydrogen was assumed in the calculations), and impose heating 1.9 to 2.7 times higher than the reference cold source. Implementation would require adding an ortho-para hydrogen converter and increasing the refrigeration capacity. The brightness increase varies considerably with energy through the cold and thermal neutron range; however, the shapes of the curves of the brightness-increase-versus-energy are very similar for all the configurations analyzed. Up to 2.3 times higher brightness is observed around $3 \AA$ wavelength. The even higher gains of 3.1 at $35 \AA$ are practically of lower interest. Meanwhile, around $1.5 \AA$ a brightness decrease of up to $50 \%$ is seen for all but one configuration. The configurations with connected HB-1 and HB-4 beamtubes allow the extraction of neutron beams from both sides of the cold source, therefore effectively doubling the potential number of instruments served, while providing brightness increases in the $40 \%$ to $90 \%$ range. A higher intensity neutron source, which would deliver $\sim 2$ times more neutrons down the beamtube, at brightness increased up to $45 \%$, can be obtained by increasing the radius of the HB- 4 and the cold source by $\sim 1 \mathrm{~cm}$; which can be realized within the restrictions imposed by the reactor vessel flanges.
\end{abstract}

\title{
1 INTRODUCTION
}

The HFIR reactor [1] will undergo a changeout of the permanent beryllium reflector in FY 2023. With the beryllium changeout, all of the beamtubes including the cold source will be replaced. The changed beryllium plug will host a suite of out-of-core irradiation ports that will be modified from the present to better meet the charge of $\mathrm{Pu}-238$ production for NASA space-flight batteries. The beryllium replacement provides an opportunity to revisit the cold source design, which is done in this report, and also to work towards an improvement of the thermal beams provided to the instruments, which is described in a separate report [2].

The four HFIR beamtubes are constrained by the core vessel flanges and reactor shielding hardwiring the axes of the beamtubes and limiting the beam tube radial dimensions. It was assumed that HB-1 and HB-3 are fixed to the present diameters, that HB-4 may expand in the beryllium section to the diameter it has in the light water section (it narrows down by about $1 \mathrm{~cm}$ in radius before entering the beryllium), and HB-2 will not expand beyond its present dimensions.

The HFIR cold source operates with liquid hydrogen at approximately $20 \mathrm{~K}$ and $1.5 \mathrm{MPa}$ and is housed in the tip of the beamtube HB-4. As no control nor measurement capability of the ortho/para state of the liquid hydrogen was included into the cryogenic loop, it can be assumed that the hydrogen is converting into a mix with large ortho content during operation. For that reason, the cold source was sized to $5 \mathrm{~cm}$ thickness to make it fairly insensitive to variation of the ortho/para ratio [3]. The ortho content is estimated to be as much as $65 \%$, based on [4], which is consistent with the estimates for the cold source at the NIST reactor [5]. 
Operating a cold source with close to $100 \%$ para hydrogen has demonstrated gains of $50 \%$ in neutron brightness at energies of $15 \mathrm{meV}$ and below compared to a moderator with $50 \%$ para hydrogen at the JPARC spallation source by increasing the moderator thickness [6]. Also, the long-pulse European Spallation Source ESS in construction [7], and the SNS PPU [8] and STS [9] projects build on moderator performance boosts to be had from para hydrogen. The reason for the marked different behaviors in neutron moderation lies in the fact that the cross section of para hydrogen drops by about two orders of magnitude below neutron energies of $20 \mathrm{meV}$ compared to the ortho hydrogen cross section as shown in Fig 1.1. How this could be exploited at the HFIR reactor cold source was the focus of this investigation. Using deuterium as cold moderator medium to benefit from its low neutron absorption cross section was also examined.

Changing the moderating medium to para hydrogen requires significant modifications to the cryogenic plant. The hydrogen volume and hence the integral heat deposition will increase and will have to be met by upgraded refrigeration systems. In addition, an ortho-to-para converter has to be integrated into the hydrogen loop. These changes are not discussed here.

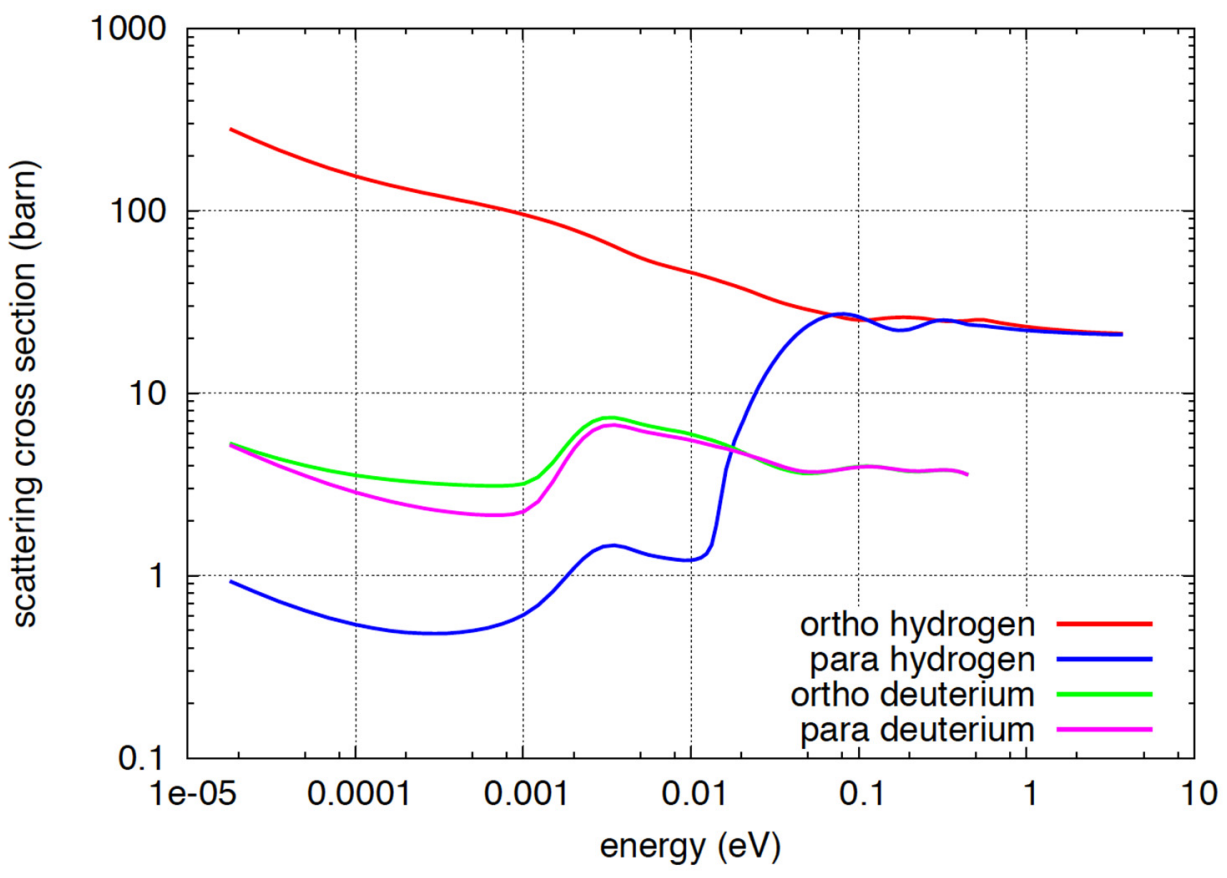

Figure 3.0.1. Comparison of scattering cross section of ortho and para hydrogen and deuterium.

Analyses were conducted to assess the cold neutron output of the cold source (CS) located in HB-4. Possible changes are limited due to the existing configuration of the pressure vessel flanges, which determine the size and location of the beam tubes. The following CS modifications were considered and the impact on the CS performance was evaluated with neutronics simulations.

- The effect of the para-hydrogen fraction on the CS brightness was examined for the existing CS configuration (present CS size and location). It was found that the CS 
brightness averaged over the full CS viewed area increases with increased para hydrogen fraction, for all neutron energy ranges of interest. (See section 3.0.)

- The CS was extended down the HB-4 in the "outward" direction. The front end of the cold source was kept at the fixed (present) location. Only $99 \%$ para hydrogen was considered as the CS material. The varied parameter was the cold source length, L, which was increased from the original $5 \mathrm{~cm}$ to over $25 \mathrm{~cm}$. The optimum value for CS length was in the range from $7 \mathrm{~cm}$ to $10 \mathrm{~cm}$ (a slight increase in length), depending on neutron energy range. At larger lengths the CS material was added in regions of lower-intensity neutron field (further away from the core) which outweighed the benefit of larger CS volume. (See Section 3.1.)

- The CS was extended inward toward HB-1 while retaining the position of the viewed CS surface, considering only a CS material of $99 \%$ para hydrogen. The varied parameter was again the cold source length, L, which was increased from the original $5 \mathrm{~cm}$ to over $25 \mathrm{~cm}$. Simultaneously the HB-1 was retracted so that the thickness of beryllium block between the HB-1 and HB-4 remained unchanged. The optimum value of $\mathrm{L}$ was found to be around $18 \mathrm{~cm}$, independent of neutron energy range. (See Section 3.2.)

- The inward extended CS described in Section 3.2 was further modified by increasing the radius of the HB-4 beam tube and the CS itself to the maximum value allowed by the vessel geometry (an increase of $\sim 1 \mathrm{~cm}$ ). As a result, the viewed area of the CS increased from $68.63 \mathrm{~cm}^{2}$ to $104.23 \mathrm{~cm}^{2}$. The varied parameter was again the CS length, L. Only 99 $\%$ para hydrogen was considered. The optimum value of $L$ was found to be around 18 $\mathrm{cm}$, independent of neutron energy range. The average brightness for this configuration was smaller than that for configuration in Section 3.2, but it was still 35\% to $45 \%$ higher than the reference CS brightness. The higher brightness and the $\sim 1.5$ times larger viewed area provided neutron source intensity that was more than the factor of two higher than the reference CS. (See Section 3.3.)

- HB-1 and HB-4 were connected, forming a "through tube" and the CS center was placed at the location closest to the reactor core. The varied parameters were the CS length, L, and para hydrogen fraction. Optimal CS length depended on the para hydrogen fraction. For the para hydrogen fraction below $\sim 90 \%$ the maximum achievable CS brightness didn't change much and the highest brightness was achieved at CS lengths in the range from $\sim 5 \mathrm{~cm}$ to $10 \mathrm{~cm}$. Big increases in brightness were observed at para-hydrogen fractions higher than $90 \%$, and the peak brightness occurred at much larger CS lengths. The CS could be viewed from the HB-4 and HB-1 side. For the $99 \%$ para-hydrogen fraction the peak brightness on the HB-4 was achieved at CS lengths in the $15 \mathrm{~cm}$ to 20 $\mathrm{cm}$ range, and a slight decrease was observed for the lengths above $20 \mathrm{~cm}$; on the HB-4 side the brightness showed small increases even at the largest CS length $(24 \mathrm{~cm})$ considered. The brightness on the HB-1 side was higher than on the HB-4 side. For the $99 \%$ para-hydrogen fraction the optimal CS length was practically independent of neutron energy range. (See Section 3.4.)

- HB-1 and HB-4 were connected, forming a "through tube" as in Section 3.4, and cold source shape modified to a cylinder with inclined viewed surfaces. The varied parameters were thickness of the CS, angle of inclination of viewed surfaces, and para-hydrogen fraction. Results largely follow the pattern observed in Section 3.4. The highest brightness was again achieved at para-hydrogen faction $99 \%$. The parameter that dominates the CS brightness was the CS length in the viewed direction (which is 
proportional to the CS volume). The peak brightness was achieved for the CS length in the viewed direction in the range $\sim 18-22 \mathrm{~cm}$. Just about the same peak brightness could be obtained with various combinations of CS thicknesses and angles of inclination, when they were combined to yield the CS length $\sim 20 \mathrm{~cm}$. The effect of inclined viewed surfaces was negligible for the CS thicknesses close to the optimal CS length. The brightnesses of the HB-4 and HB-1 side were about equal, which was different from the Section 3.4. A weak dependence of optimal CS length on the energy range on the HB-4 side was observed. (See Section 3.5.)

- For the CS with increased radius and extended inwards, described in Section 3.3, liquid hydrogen was replaced by liquid deuterium. The resulting brightness was lower that the brightness of the reference CS and the CS filled with $99 \%$ para-hydrogen. Therefore, even the largest possible CS geometry permitted by the beam tube constraints does not benefit from the use of deuterium as a moderating material (See Section 3.3.)

\section{CALCULATIONAL METHODOLOGY}

Transport calculations were performed by MCNPX version 2.7.0 [10] with a model named HF27N0 claimed to be a simplified version of James Bucholz's model HF245 [3] and the core in EOL conditions as built and used by Peplow and Xoubi [11,12]. The thermal scattering kernels of ENDF/B-VII were applied for hydrogen (hpara.10t and hortho.10t from endf70sab). The model was instrumented with parameters, so that changes such as axial movement, or the length of the cold source could be propagated into the model by the PSTUDY [13] tool through user provided variable settings.

Furthermore, point detectors were established in the beam tubes at 6 meters distance from the location of interest for the brightness assessment of the viewed face(s) of the cold source, unless otherwise stated. Also, collimators were established in the beamtubes such that only neutrons from the location of interest for brightness assessment were allowed to contribute to the point detector.

In order to speed up the analysis, two measures were taken. Firstly, the reflector zones around the beam tube noses were separated out from the bulk reflector and used as contributing zones to the point detector neutron flux estimate, while the bulk was excluded. Secondly, the ADVANTG code [14] was used to calculate weight window parameters for a mesh-based variance reduction scheme in order to focus the calculational effort to the zones mostly contributing to the analysis results.

\section{COLD SOURCE INVESTIGATIONS}

\subsection{PRESENT COLD SOURCE CONFIGURATION}

A sketch of the existing cold source (CS) vessel is shown in Fig. 3.0.1. It consists of an aluminum capsule with spherical dome on the end, cylindrical body, and reentrant concave dome for the viewed surface. The capsule has an outer diameter of $9.84 \mathrm{~cm}$ and the length of $\sim 5 \mathrm{~cm}$. 
The volume of the CS capsule is $627 \mathrm{~cm}^{3}$; aluminum volume is $162 \mathrm{~cm}^{3}$, and hydrogen volume is $465 \mathrm{~cm}^{3}$.

The CS is currently installed in the HB-4, as illustrated in Fig. 3.0.2. The beam tubes HB-4 and HB-1 are not connected and the space between them is occupied by beryllium reflector.

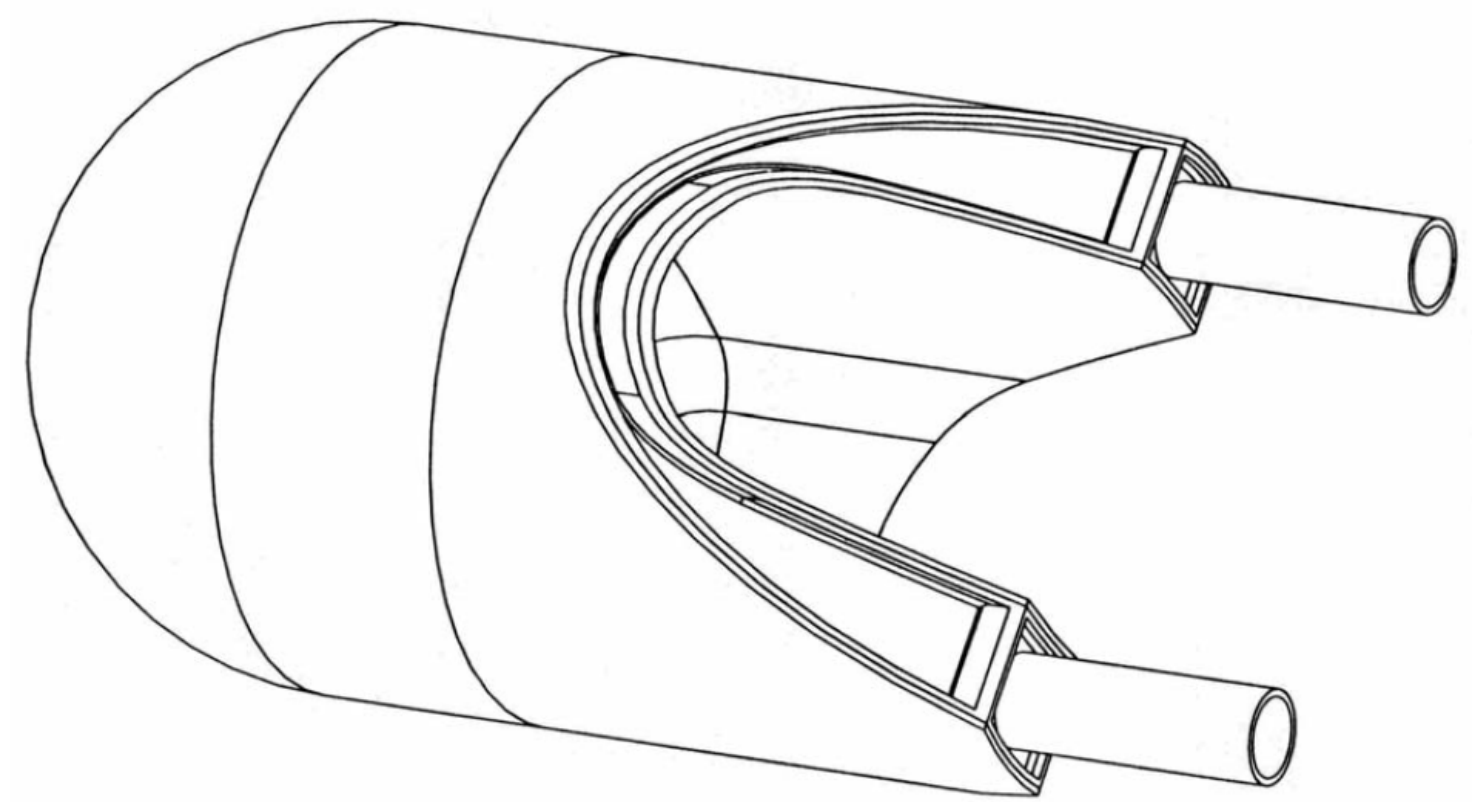

Figure 3.0.1. Sketch of the present configuration of the HFIR cold source. (Reproduced from [3]) 

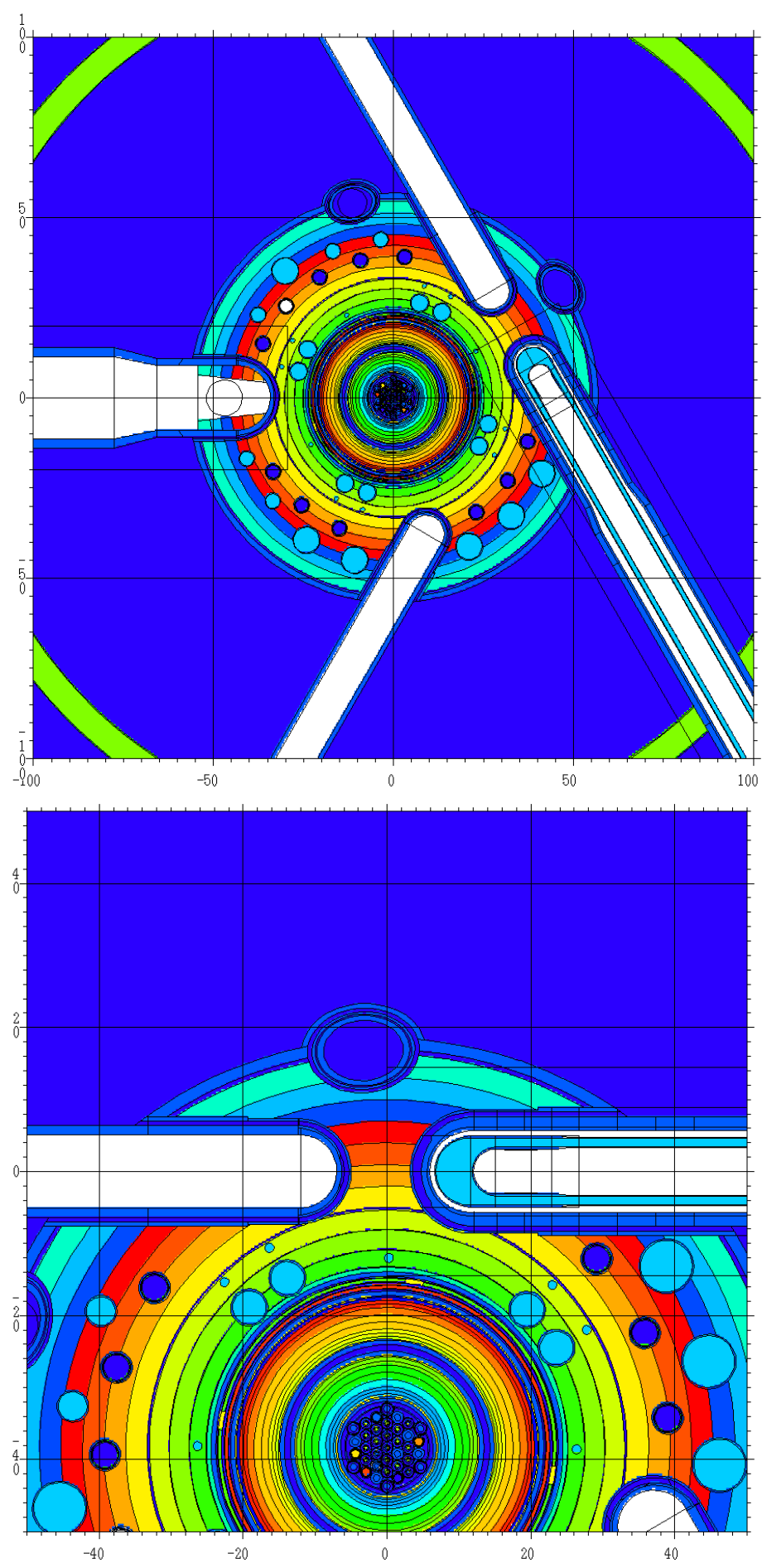

Figure 3.0.2

Figure 3.0.2. Horizontal section through the MCNP model of the HFIR reactor (top); detail of the region around the tip of the HB-1 and HB-4, with the cold source (bottom). Colors indicate different materials. 
The CS is filled with liquid hydrogen at temperature $\sim 20^{\circ} \mathrm{K}$, pressure $\sim 1.5 \mathrm{MPa}$. The cryogenic hydrogen loop at HFIR does not include a catalytic converter and the actual ortho-para hydrogen ratio is not monitored and not known.

Five neutron scattering instruments, currently installed on HFIR CS, are listed in Table 3.0.1, together with their operating ranges of neutron energies and wavelengths.

Table 3.0.1. Neutron scattering instruments on HFIR cold source and corresponding neutron energies and wavelengths of interest.

\begin{tabular}{|c|c|c|c|c|}
\hline \multicolumn{3}{|c|}{ Instruments on HFIR Cold Source } & $\begin{array}{l}\text { Energy } \\
\text { Range } \\
(\mathrm{meV})\end{array}$ & $\begin{array}{c}\text { Wavelength } \\
\text { Range } \\
(\AA)\end{array}$ \\
\hline $\begin{array}{l}\text { Neutron Imaging Facility } \\
\text { General-Purpose Small-Angle }\end{array}$ & IMAGING & CG-1D & $2.3-25$ & $6.0-1.8$ \\
\hline $\begin{array}{l}\text { Neutron Scattering } \\
\text { Diffractometer }\end{array}$ & GP-SANS & CG-2 & $0.13-5.1$ & $25-4.0$ \\
\hline $\begin{array}{l}\text { Biological Small-Angle } \\
\text { Neutron Scattering Instrument }\end{array}$ & BIO-SANS & CG-3 & $0.13-2.3$ & $25-6.0$ \\
\hline $\begin{array}{l}\text { Cold Neutron Triple-Axis } \\
\text { Spectrometer }\end{array}$ & CTAX & CG-C4 & $2-20$ & $6.4-2.0$ \\
\hline Laue Diffractometer & IMAGINE & CG-4D & $4-20$ & $4.5-2.0$ \\
\hline
\end{tabular}

Table 3.0.2 gives the calculated CS brightness for two para-hydrogen fractions. The values are for the existing CS configuration (present CS size and location), averaged over a viewed area $68.63 \mathrm{~cm}^{2}$ (the full CS), and are normalized to the HFIR core power of $85 \mathrm{MW}$. The brightness was calculated for five energy ranges which are of interest for the instruments currently installed at the HB-4 beam tube (see Table 3.0.1).

Fig. 3.0.3 shows the calculated CS brightness as a function of para-hydrogen fraction over the full range of para-hydrogen fractions. The hydrogen density was kept constant at $0.0726 \mathrm{~g} \mathrm{~cm}^{-3}$ in all calculations. The brightness is normalized to the brightness at $35 \%$ para-hydrogen fraction. For para-hydrogen fraction below $\sim 70 \%$, the brightness for all energy intervals shows little variation. For para-H fraction above $70 \%$, the brightness varies more significantly and is as much as $35 \%$ higher at $99 \%$ para-hydrogen fraction. The brightness in all energy ranges is the highest for the highest para-H fraction.

Detailed variation of CS brightness with neutron energy is shown in Fig. 3.0.4 for the full energy range and in Fig. 3.0.5 for narrower energy range of interest for the CS.

The present CS is very likely operated with a para fraction of below $70 \%$ and is dimensioned such that its performance is insensitive to variations of the para fraction.

The brightness of the existing CS (present CS size and location) with viewed area $68.63 \mathrm{~cm}^{2}$ (the full CS), and $35 \%$ para-hydrogen fraction was selected as the CS configuration to which other CS configurations will be compared in this analysis. This CS configuration and its brightness 
will be referred to as the "reference CS" and the "reference brightness" or just simply "reference" for short.

Table 3.0.2. CS brightness for the existing CS (present CS size and location), viewed area $68.63 \mathrm{~cm}^{2}\left(\mathrm{the}_{\mathrm{full}}\right.$ CS), and two para-hydrogen fractions, in selected energy ranges. Brightness is given for the HFIR core power 85 MW.

\begin{tabular}{|c|c|c|c|c|c|}
\hline & \multicolumn{5}{|c|}{$\begin{array}{c}\text { Cold Source Brightness } \\
\text { (neutrons } / \mathrm{cm}^{2} / \mathrm{s} / \mathrm{sr} / \mathrm{eV} \text { ) }\end{array}$} \\
\hline & $\begin{array}{c}0.13-2.3 \\
\mathrm{meV} \\
25.1-6 \AA\end{array}$ & $\begin{array}{c}0.13-5.1 \\
\mathrm{meV} \\
25.1-4 \AA\end{array}$ & $\begin{array}{c}2.0-20.0 \\
\mathrm{meV} \\
6.4-2.0 \AA\end{array}$ & $\begin{array}{c}2.3-25.0 \\
\mathrm{meV} \\
6.0-1.8 \AA\end{array}$ & $\begin{array}{c}4.0-20.0 \\
\mathrm{meV} \\
4.5-2.0 \AA\end{array}$ \\
\hline Para-H $35 \%$ & $\begin{array}{l}1.21 \mathrm{E}+15 \\
(0.39 \%)^{\mathrm{a}}\end{array}$ & $\begin{array}{c}1.63 \mathrm{E}+15 \\
(0.28 \%)\end{array}$ & $\begin{array}{c}1.19 \mathrm{E}+15 \\
(0.22 \%)\end{array}$ & $\begin{array}{l}1.01 \mathrm{E}+15 \\
(0.22 \%)\end{array}$ & $\begin{array}{c}1.09 \mathrm{E}+15 \\
(0.23 \%)\end{array}$ \\
\hline \multirow[t]{3}{*}{ Para-H 99 \% } & $\begin{array}{l}1.30 \mathrm{E}+15 \\
(0.34 \%)^{\mathrm{a}}\end{array}$ & $\begin{array}{c}1.71 \mathrm{E}+15 \\
(0.22 \%)\end{array}$ & $\begin{array}{c}1.53 \mathrm{E}+15 \\
(0.17 \%)\end{array}$ & $\begin{array}{l}1.28 \mathrm{E}+15 \\
(0.17 \%)\end{array}$ & $\begin{array}{c}1.48 \mathrm{E}+15 \\
(0.18 \%)\end{array}$ \\
\hline & \multicolumn{5}{|c|}{$\begin{array}{l}\text { Cold Source Brightness } \\
\text { (neutrons } / \mathrm{cm}^{2} / \mathrm{s} / \mathrm{sr} / \AA \text { ) }\end{array}$} \\
\hline & $\begin{array}{c}0.13-2.3 \\
\text { meV } \\
25.1-6 \AA\end{array}$ & $\begin{array}{c}0.13-5.1 \\
\text { meV } \\
25.1-4 \AA\end{array}$ & $\begin{array}{c}2.0-20.0 \\
\mathrm{meV} \\
6.4-2.0 \AA\end{array}$ & $\begin{array}{c}2.3-25.0 \\
\mathrm{meV} \\
6.0-1.8 \AA \\
\end{array}$ & $\begin{array}{c}4.0-20.0 \\
\mathrm{meV} \\
4.5-2.0 \AA\end{array}$ \\
\hline Para-H $35 \%$ & $1.37 \mathrm{E}+11$ & $3.85 \mathrm{E}+11$ & $4.90 \mathrm{E}+12$ & $5.49 \mathrm{E}+12$ & $7.01 \mathrm{E}+12$ \\
\hline Para-H $99 \%$ & $1.47 \mathrm{E}+11$ & $4.04 \mathrm{E}+11$ & $6.30 \mathrm{E}+12$ & $6.97 \mathrm{E}+12$ & $9.45 \mathrm{E}+12$ \\
\hline
\end{tabular}

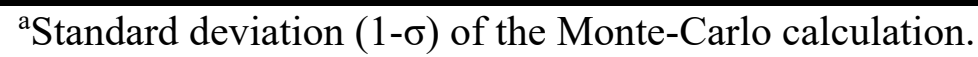

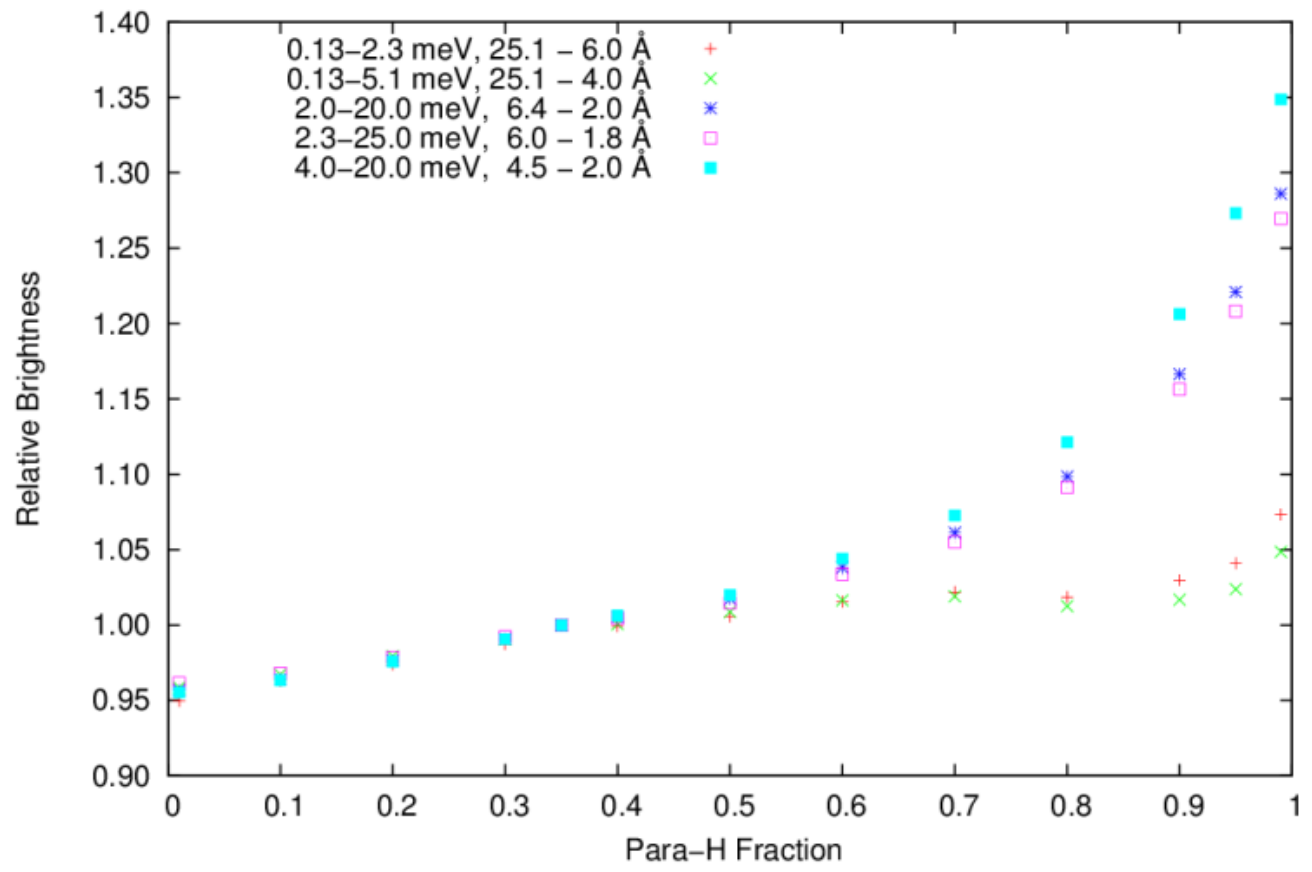

Figure 3.0.3. Brightness of the exiting CS, averaged over the viewed area $\left(68.63 \mathrm{~cm}^{2}\right.$, the full $\left.\mathrm{CS}\right)$ as a function of para-hydrogen fraction. 


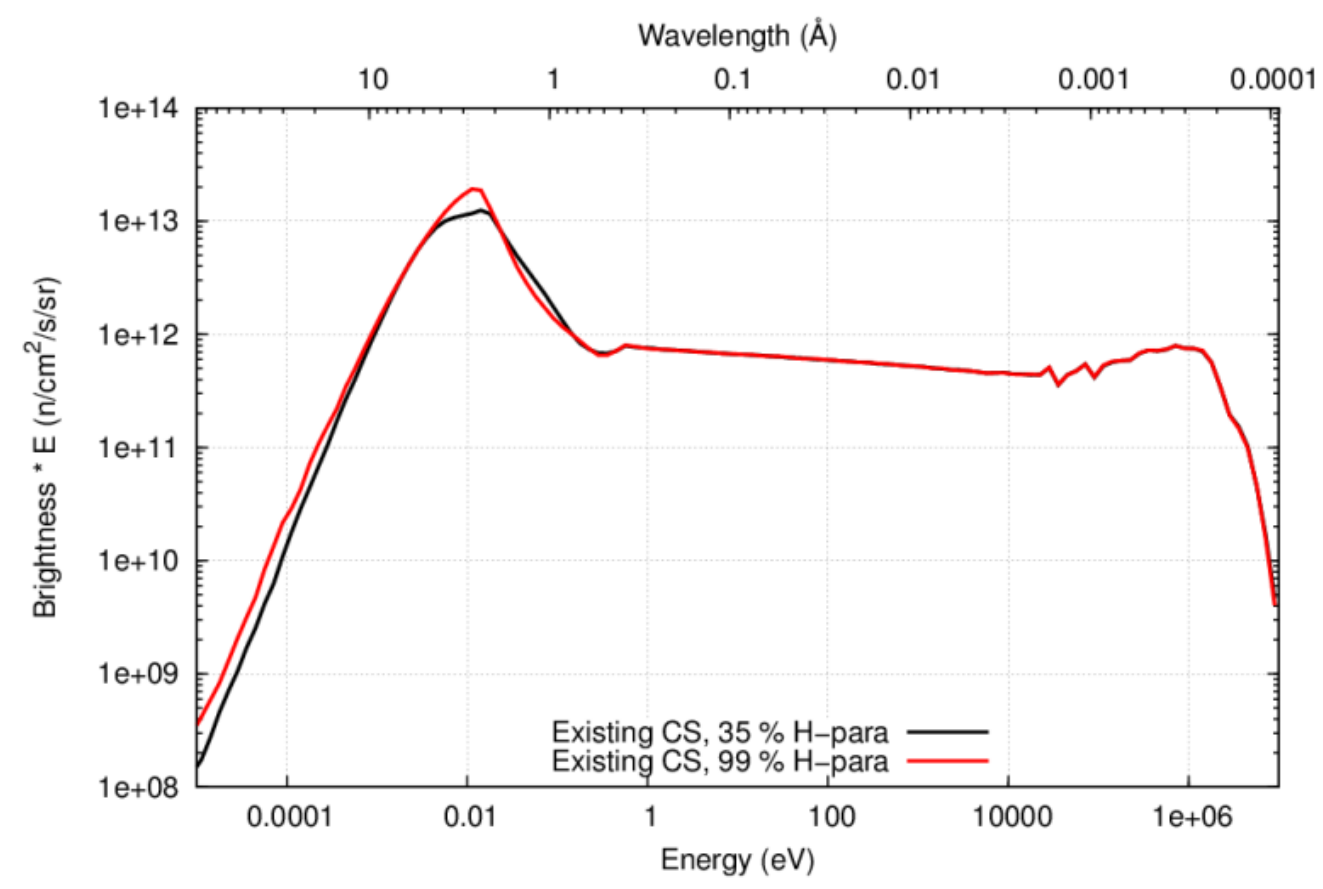

Figure 3.0.4. Brightness versus neutron energy (full energy range), for the existing CS configuration, actual location, viewed area $68.63 \mathrm{~cm}^{2}$ (the full CS), and two para-hydrogen fractions.

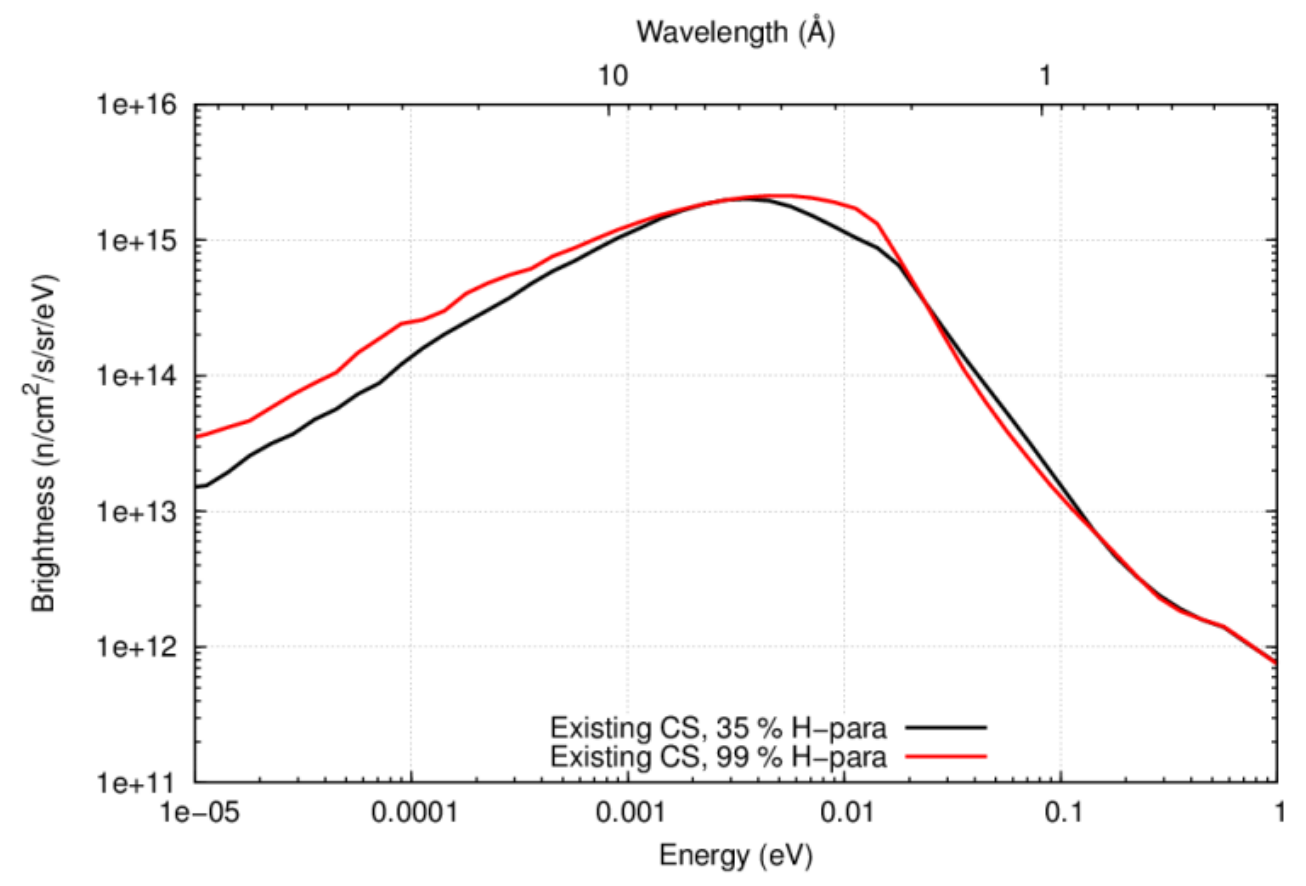

Figure 3.0.5. Brightness versus neutron energy (below $1 \mathrm{eV}$ ), for the existing CS configuration, actual location, viewed area $68.63 \mathrm{~cm}^{2}$ (the full CS), and two para-hydrogen fractions. 


\subsection{COLD SOURCE EXTENDED OUTWARD INTO HB-4 AT PRESENT CS RADIUS}

The first modification to the CS considered was the extension of the CS length outwards into HB-4 (see Fig. 3.1.1). The highest brightness is achieved at CS lengths in the range of $7 \mathrm{~cm}$ to 10 $\mathrm{cm}$ as shown in Fig. 3.1.2. The brightness increases by $\sim 15-20 \%$ for the longer wavelengths and $\sim 30-40 \%$ for the shorter wavelengths. The main reason that this configuration results in relatively modest increase in brightness is that the CS volume is extended outwards into the region with lower neutron flux. The para-hydrogen content was $99 \%$ in all calculations shown in Fig 3.1.2.

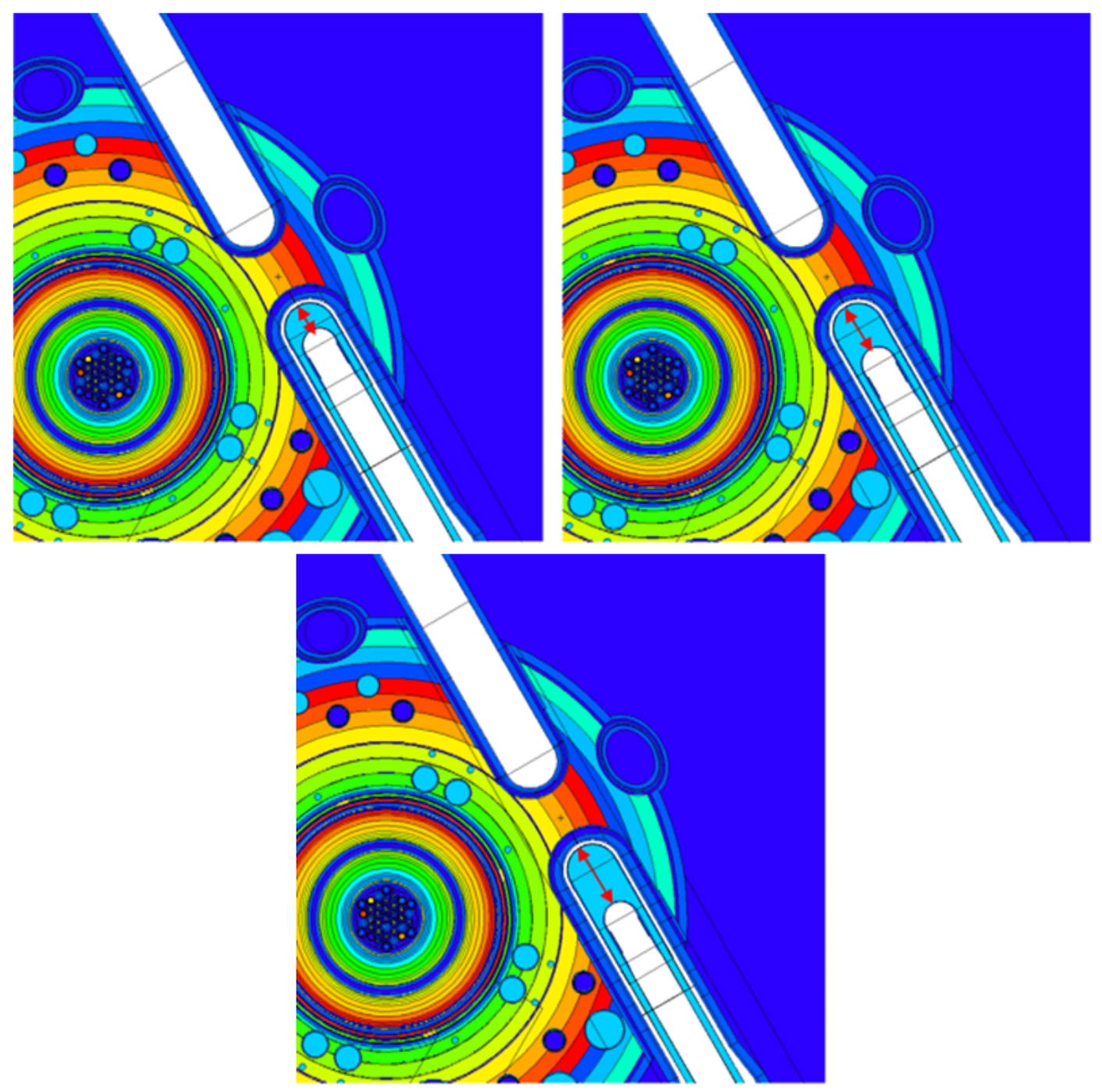

Figure 3.1.1. Cold source configurations of existing design extending in length in the outward direction. The CS length (L) is indicated by the red arrow. The CS tip (front end) stays at the fixed location and the CS is extended in the outward direction (down the HB-4 beamtube). Only the initial and two configurations with extended CS are shown. 


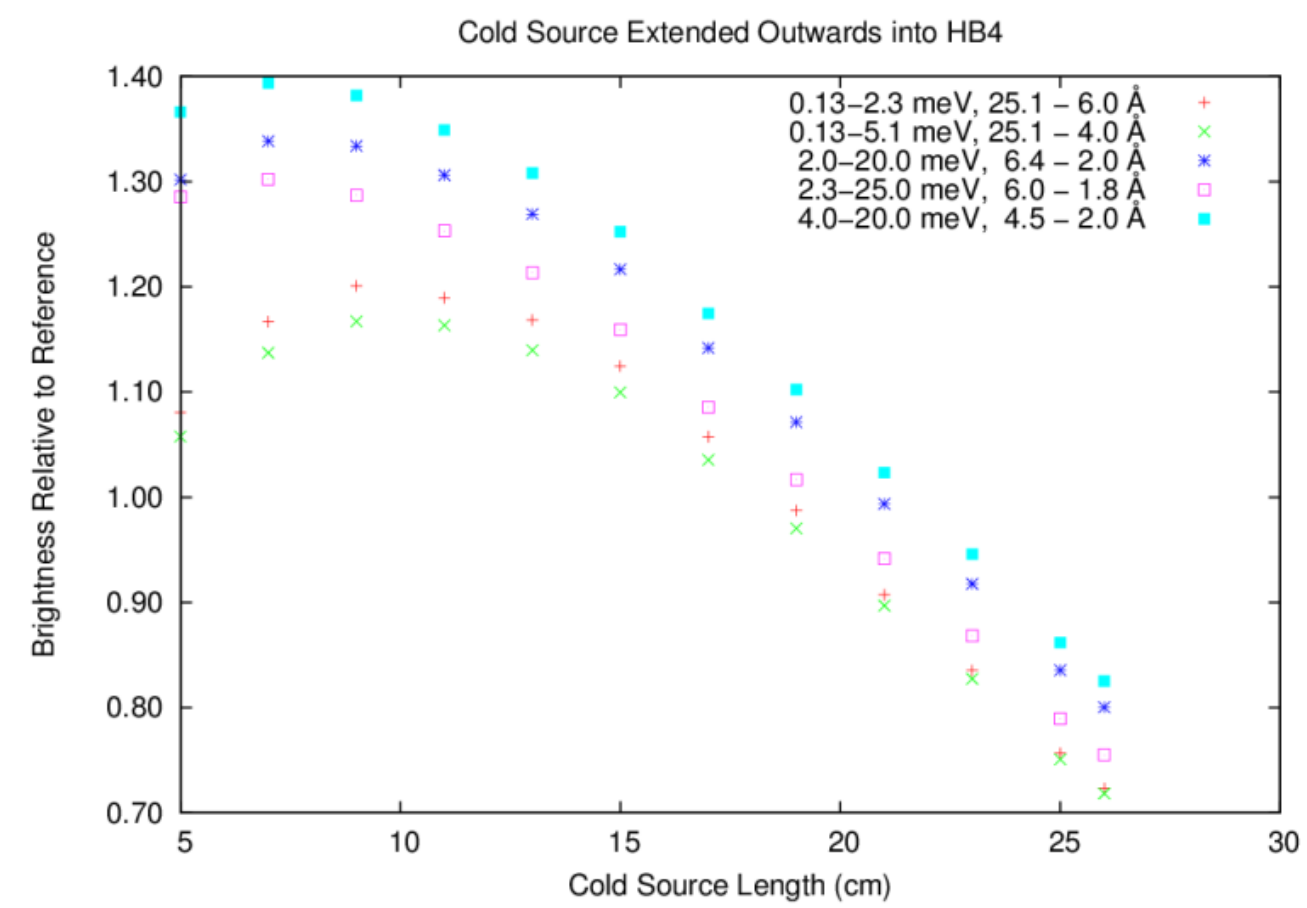

Figure 3.1.2. Brightness versus CS length, CS is extended outwards into the HB-4. The brightness is given relative to the brightness of the reference CS. The viewed area is $68.63 \mathrm{~cm}^{2}$. Para-hydrogen fraction $t$ is $99 \%$.

\subsection{COLD SOURCE EXTENDED INWARD INTO HB-4 AT PRESENT CS RADIUS}

A more promising way to extend the CS is inwards (towards the HB-1, see Fig. 3.2.1) closer to the core into the region with higher neutron flux; again, $99 \%$ para-hydrogen was used. The CS brightness relative to the brightness of the reference CS is shown in Fig. 3.2.2. Substantial gains in brightness, in the order of $45 \%$ to $65 \%$ are observed for the CS lengths of $\sim 20 \mathrm{~cm}$ or more.

The HB-1 tube was simultaneousely retracted by the same length as the CS was extended, so that the thickness of the beryllium block between the HB-1 and HB-4 remained constant. This has a negative effect on the HB-1, because the displacement of HB-1 in the outward direction results in losses of thermal fluxes at HB-1 as shown in Section 3.6. The option of not moving the HB-1 and reducing the beryllium thickness between HB-1 and HB-4 was not studied, since the emphasis was on the CS performance. This option may be considered in the future if it becomes of interest. 

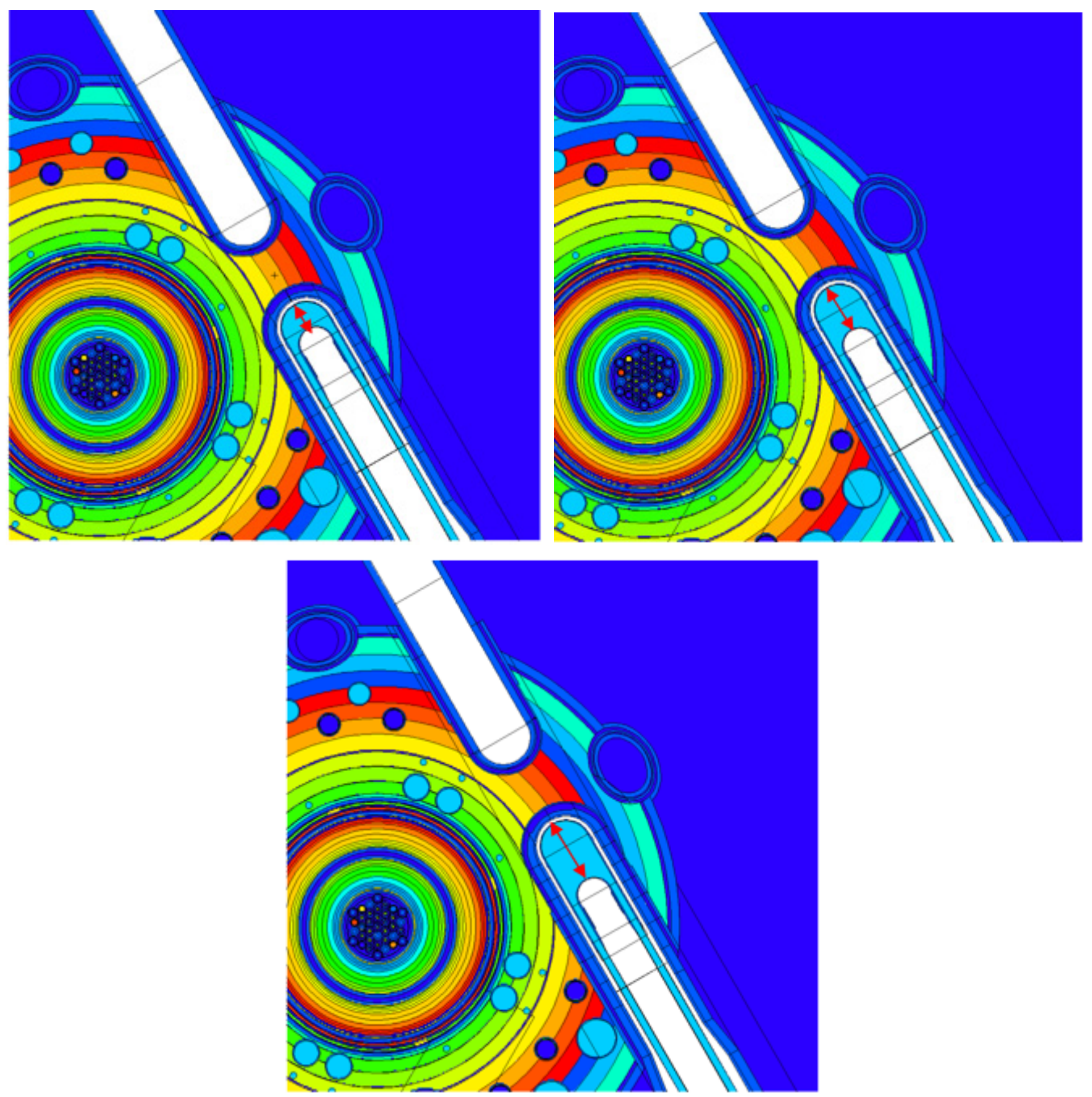

Figure 3.2.1. Cold source configurations of existing design extending in length in the inward direction. The CS length $(L)$ is indicated by the red arrow. The viewed surface of the CS is at fixed location and the CS is extended in the inward direction (towards the HB-1). The HB-1 tube is simultaneousely retracted by the same amount, so that the thickness of the beryllium block between the HB-1 and HB-4 remains constant. Only the initial, and two configurations with extended CS are shown. 


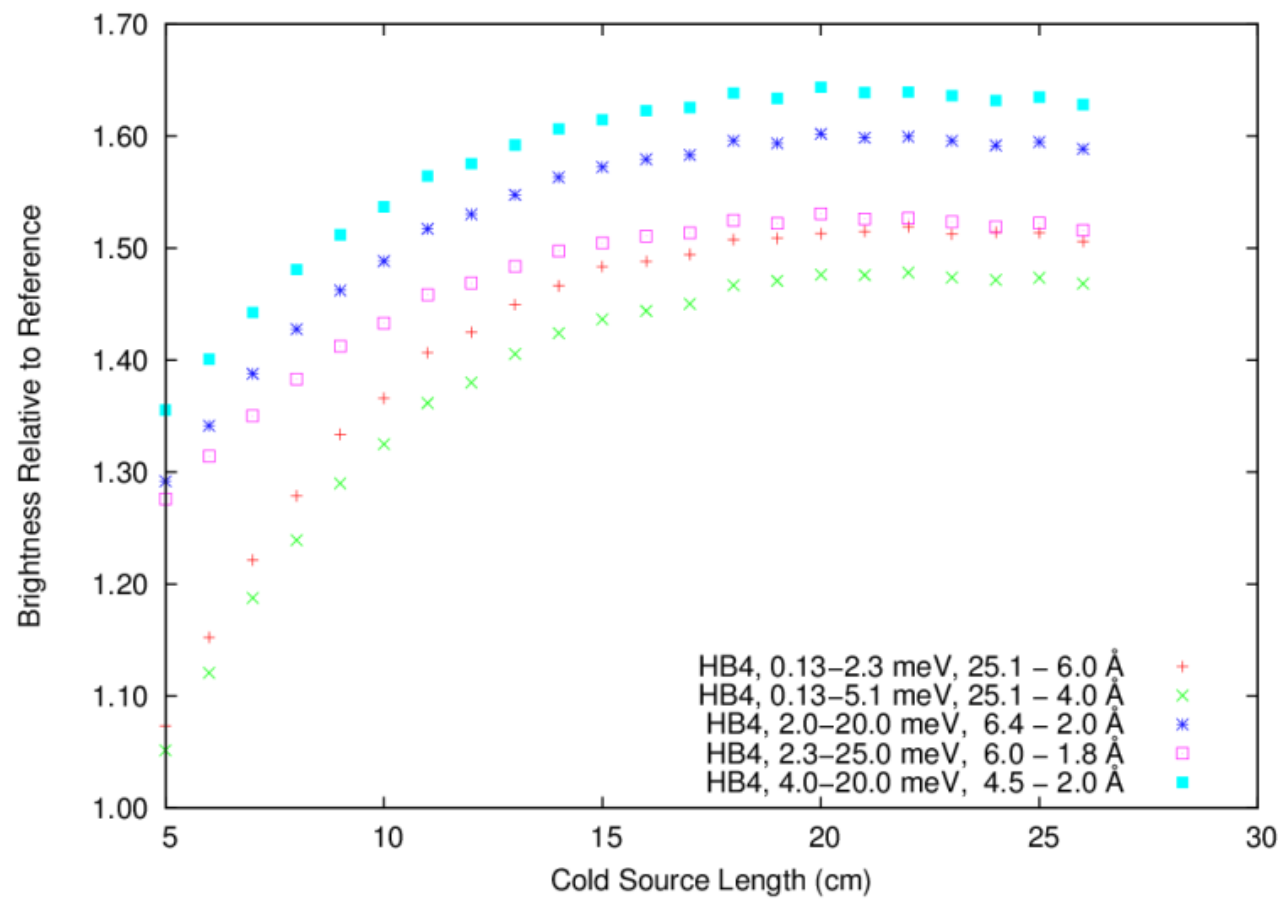

Figure 3.2.2. Brightness versus CS length, CS is extended towards HB-1 and HB-1 is retracted by the same length. The brightness is given relative to the brightness of the reference CS. The viewed area is $68.63 \mathrm{~cm}^{2}$. Para-hydrogen fraction is $99 \%$.

\subsection{COLD SOURCE EXTENDED INWARD INTO HB-4 AND INCREASED IN RADIUS}

The existing HB-4 beam tube is slightly reduced in radius just before HB-4 enters the beryllium reflector. In the CS configuration considered in this section this reduction was eliminated. This increases the radius of the inner part of the HB-4 by $\sim 1 \mathrm{~cm}$, so that the HB-4 maintains the same radius along the full length within the core vessel. The CS radius was also increased by the same amount. Because of this change two vertical irradiation facilities, VXF-21 and VXF-22, that were the closest to the HB-4, had to be moved slightly away from HB-4. Again, the CS length was increased in the inward direction as described in the section 3.2 (see Fig. 3.3.1) and the hydrogen was $99 \%$ para-hydrogen.

The brightness for these cases compared to the reference CS brightness is shown in Fig. 3.3.2. The brightness in all energy intervals increases with increasing CS length until $\sim 20 \mathrm{~cm}$; for larger lengths, there is no further increase. The increase in brightness is in the range from $35 \%$ to $45 \%$. The higher brightness and the $\sim 1.5$ times larger viewed area provide neutron source intensity that is more than the factor of two higher than the reference CS. 

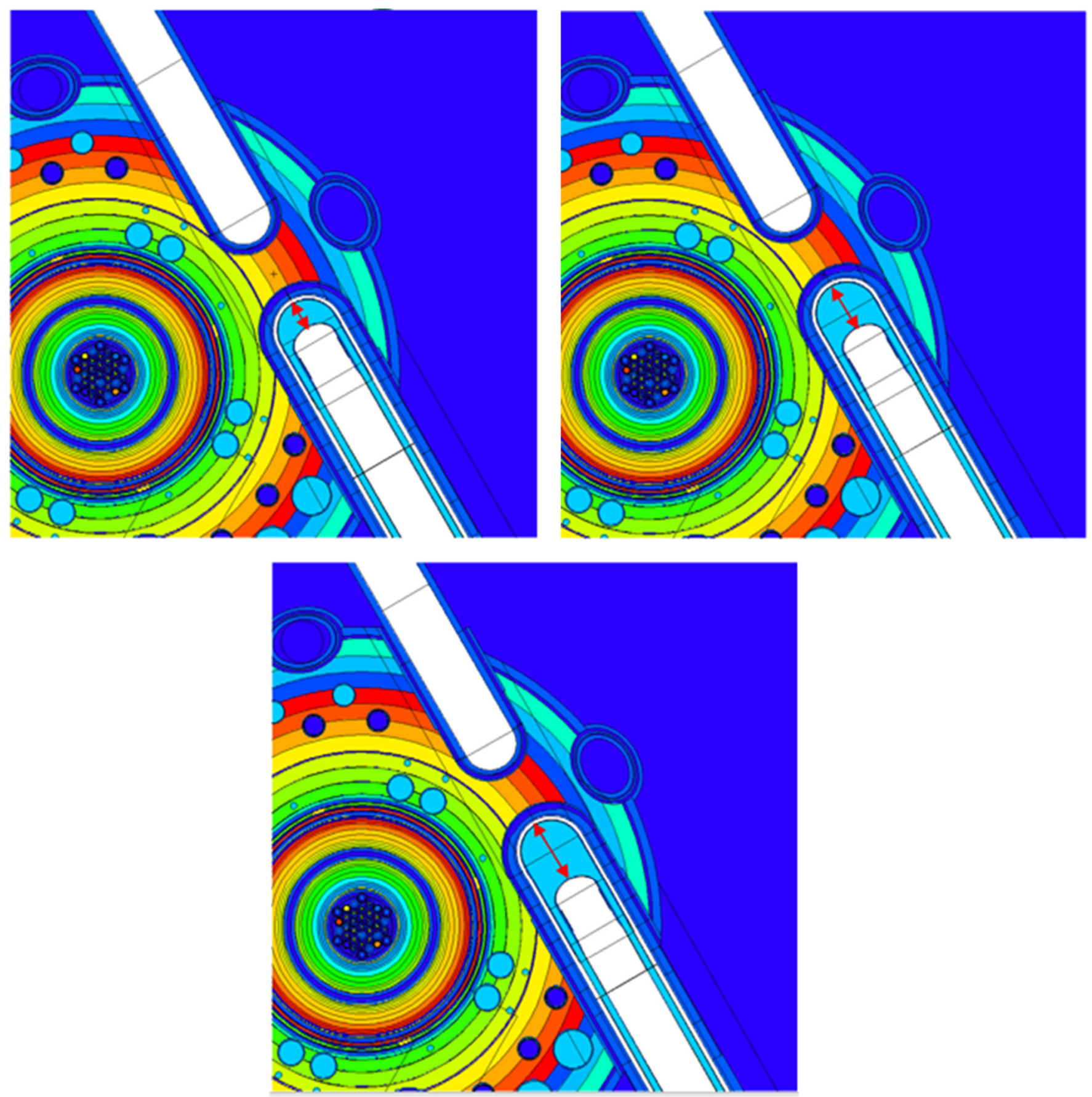

Figure 3.3.1. Cold source configurations of existing design but beamtube and CS radii enlarged by $1 \mathrm{~cm}$ and extending in length in the inward direction. The CS length $(L)$ is indicated by the red arrow. The viewed surface of the CS is at fixed location and the CS is extended in the inward direction (towards the HB-1). The HB-1 tube is simultaneousely retracted by the same amount so that the thickness of the beryllium block between the HB-1 and HB-4 remains constant. Only the initial and two configurations with extended CS are shown. 


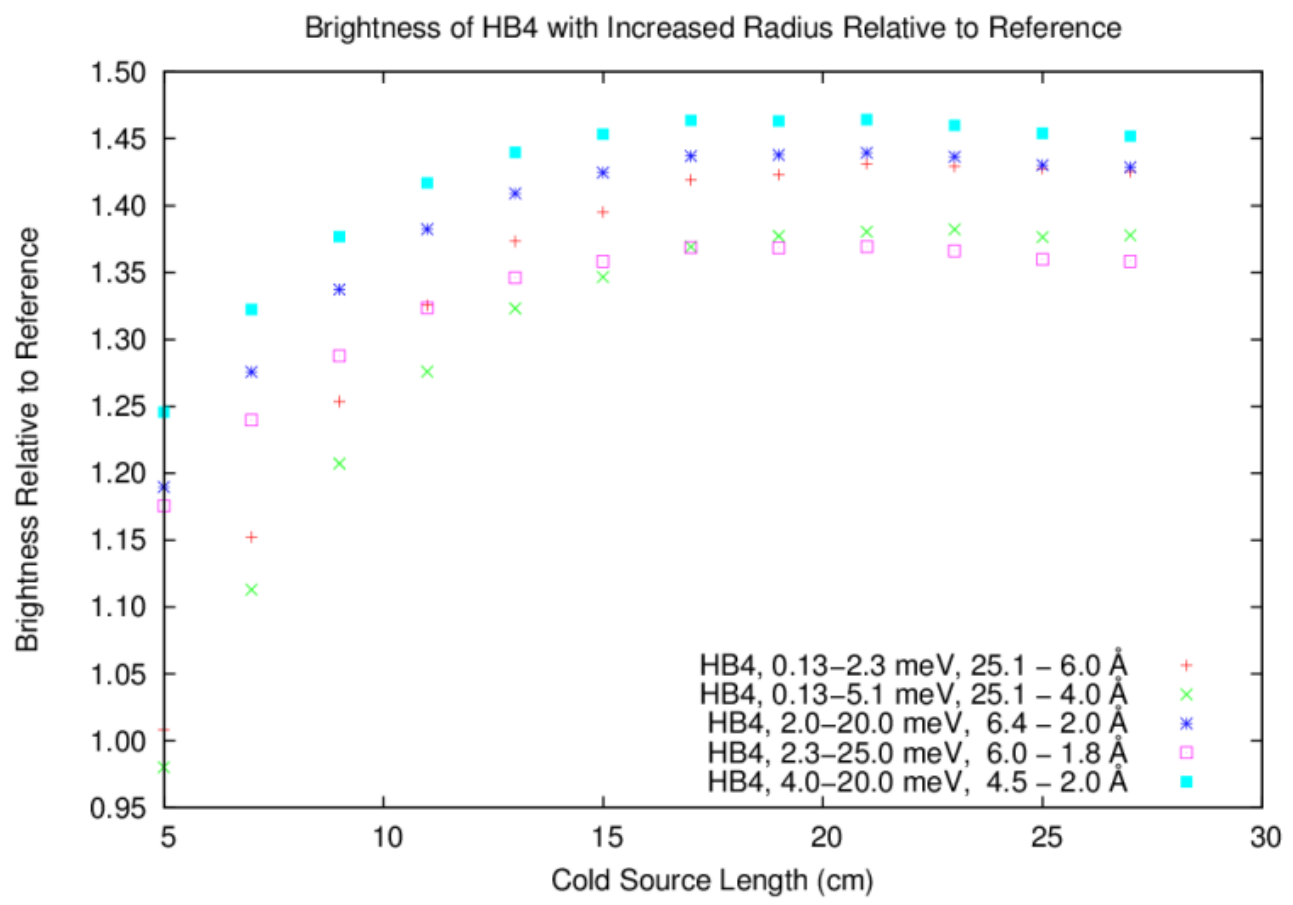

Figure 3.3.2. Brightness versus CS length with CS increased in radius by $\sim 1 \mathrm{~cm}$ (viewed area is $104.23 \mathrm{~cm}^{2}$ ) and extended towards HB-1. HB-1 is retracted by the CS length increase. The brightness is given relative to the brightness of the reference CS. Para hydrogen fraction is $99 \%$.

In the CS configurations with increased radius, discussed above, the hydrogen was replaced with deuterium, and the CS brightness was recalculated. The deuterium composition was $25 \%$ paradeuterium and $75 \%$ ortho-deuterium, at the temperature of $20 \mathrm{~K}$, pressure $1 \mathrm{MPa}$, and density $0.1733 \mathrm{~g} \mathrm{~cm}^{-3}$ ( $\mathrm{S}_{\mathrm{ab}}$ data were from ENDF/B-VII, dpara.10t and dortho.10t, at $19 \mathrm{~K}$ ). The results are shown in Fig. 3.3.3. The brightness of the CS filled with deuterium is at least $15 \%$ smaller than the brightness of the reference CS. This indicates that for a CS that is restricted to the volume available inside the beam tube, the use of deuterium moderator is not an advantage.

Detailed brightness versus neutron energy plots for the reference CS, and extended CS with length $17 \mathrm{~cm}$ and increased radius are shown in Fig. 3.3.4 and 3.3.5. For the extended CS with increased radius the brightness is shown for the CS filled with hydrogen (99\% para-hydrogen) and with deuterium ( $75 \%$ ortho-deuterium and $98 \%$ ortho-deuterium). Due to small difference in cross-sections for para- and ortho-deuterium, the curves for the two ortho-deuterium fractions practically overlap. The peak brightness for the CS filled with deuterium is lower than that of hydrogen. Deuterium gives higher brightness at wavelengths longer than $\sim 30 \AA$ and at wavelengths shorter than $\sim 2 \AA$. 


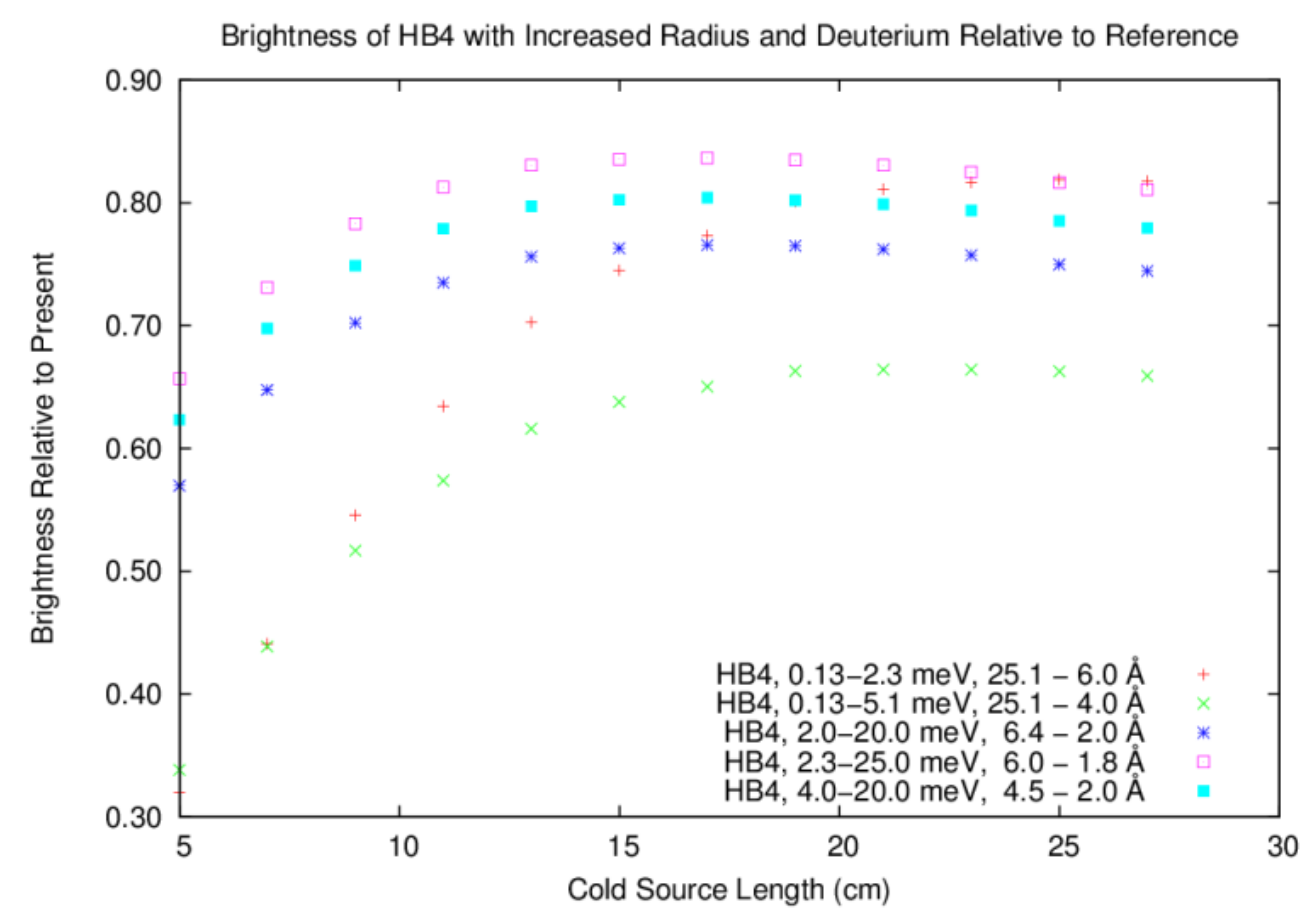

Figure 3.3.3. Brightness versus CS length with deuterium filled CS increased in radius by $\sim 1 \mathrm{~cm}$ (viewed area is $104.23 \mathrm{~cm}^{2}$ ) and extended towards the HB-1. HB-1 is retracted by the same length. The brightness is given relative to the brightness of the reference CS. CS is filled with deuterium ( $75 \%$ ortho-deuterium).

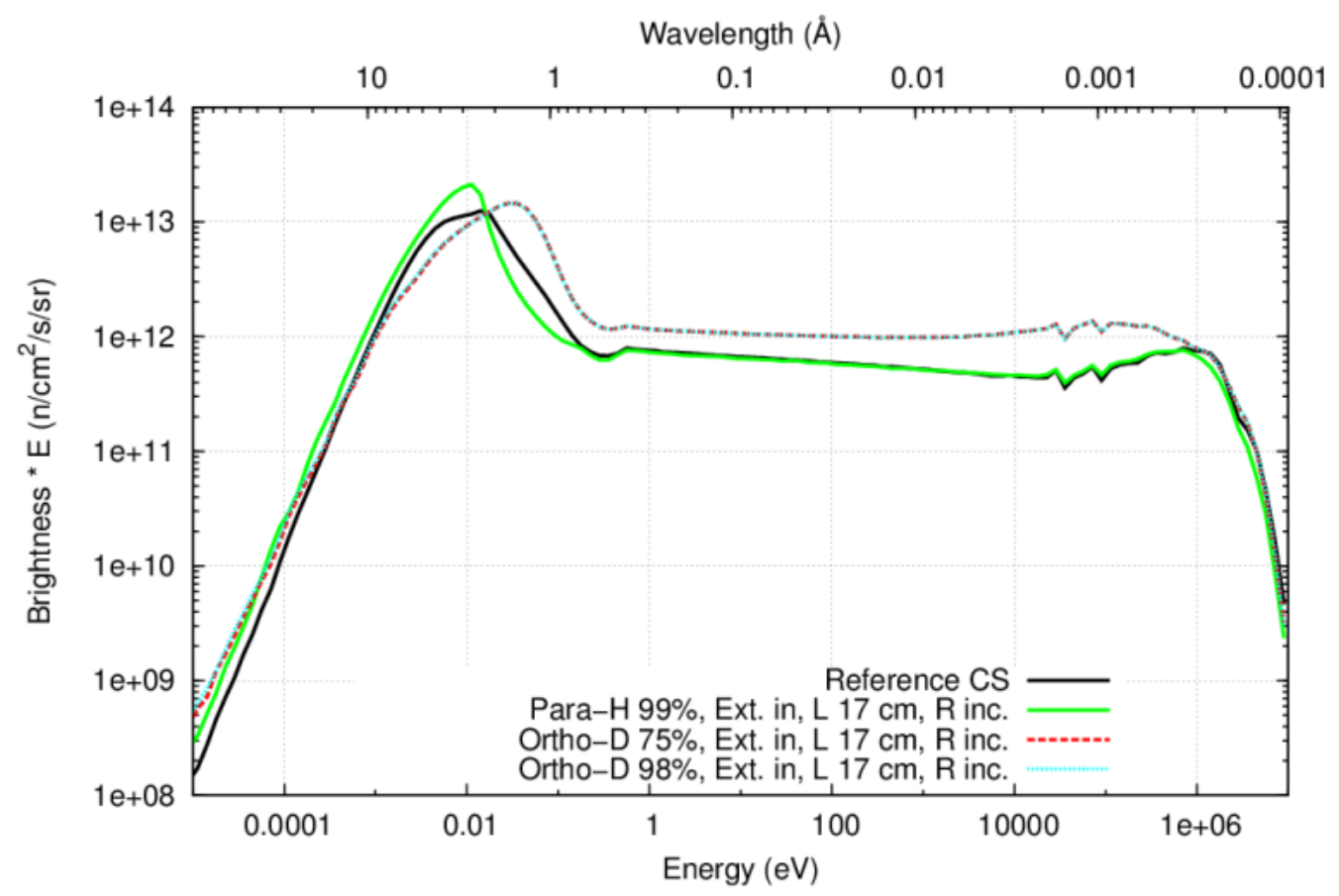

Figure 3.3.4. Brightness versus neutron energy (full energy range) for the reference CS, and extended CS (length $17 \mathrm{~cm}$ ) with increased radius, filled with hydrogen and deuterium. 


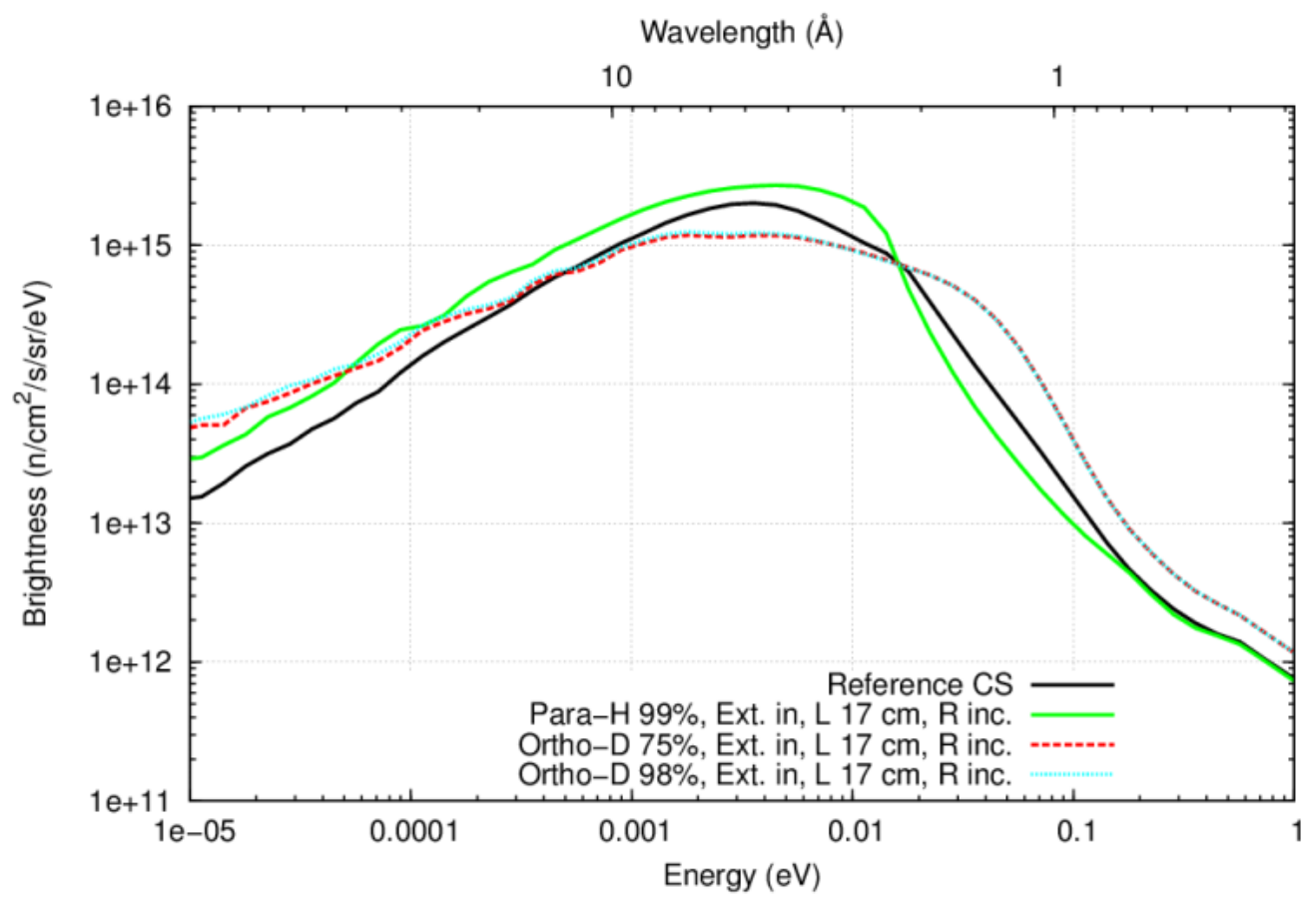

Figure 3.3.5. Brightness versus neutron energy (for energies below $1 \mathrm{eV}$ ), for the reference CS, and extended CS (length $17 \mathrm{~cm}$ ) with increased radius, filled with hydrogen and deuterium.

\subsection{HB-1 AND HB-4 CONNECTED, COLD SOURCE CENTERED, AND LENGTH EXTENDED}

In this section, the HB-1 and HB-4 beam tubes are assumed connected and the CS center is placed at the location closest to the reactor core as shown in Fig. 3.4.1. Numerous calculations were performed for this configuration to study the CS brightness as a function of the CS length and the hydrogen ortho/para fraction. Some results are depicted in Fig. 3.4.2. For the parahydrogen fraction below $\sim 90 \%$ the CS brightness doesn't change much, as shown in Fig. 3.4.3. For this range of para-hydrogen fraction, the highest brightness is achieved at CS lengths in the range from $\sim 5 \mathrm{~cm}$ to $10 \mathrm{~cm}$ and showing brightness decreases with further increase in the CS length. Big increases in brightness are observed at para-hydrogen fractions higher than 90\%, and the peak brightness occurs at much larger CS lengths. For the 99\% para-hydrogen fraction the peak brightness is achieved at CS lengths in the $15 \mathrm{~cm}$ to $20 \mathrm{~cm}$ range, and a slight decrease is observed for the lengths above $20 \mathrm{~cm}$.

In this configuration, the CS can be viewed both from the HB-4 and HB-1 side, which effectively doubles the potential number of instrument locations on the CS. Brightness in different energy ranges as a function of CS length, for the $99 \%$ para-hydrogen fraction is shown in Fig. 3.4.4, as viewed from the HB-4 side, and in Fig. 3.4.5, as viewed from the HB-1 side. The increase in brightness is $\sim 40 \%$ to $\sim 55 \%$ on the HB- 4 side and $70 \%$ to $85 \%$ on the HB- 1 side with respect to the reference CS brightness. The brightness on the HB-1 side shows increasing trend up to the longest lengths of the CS considered. 

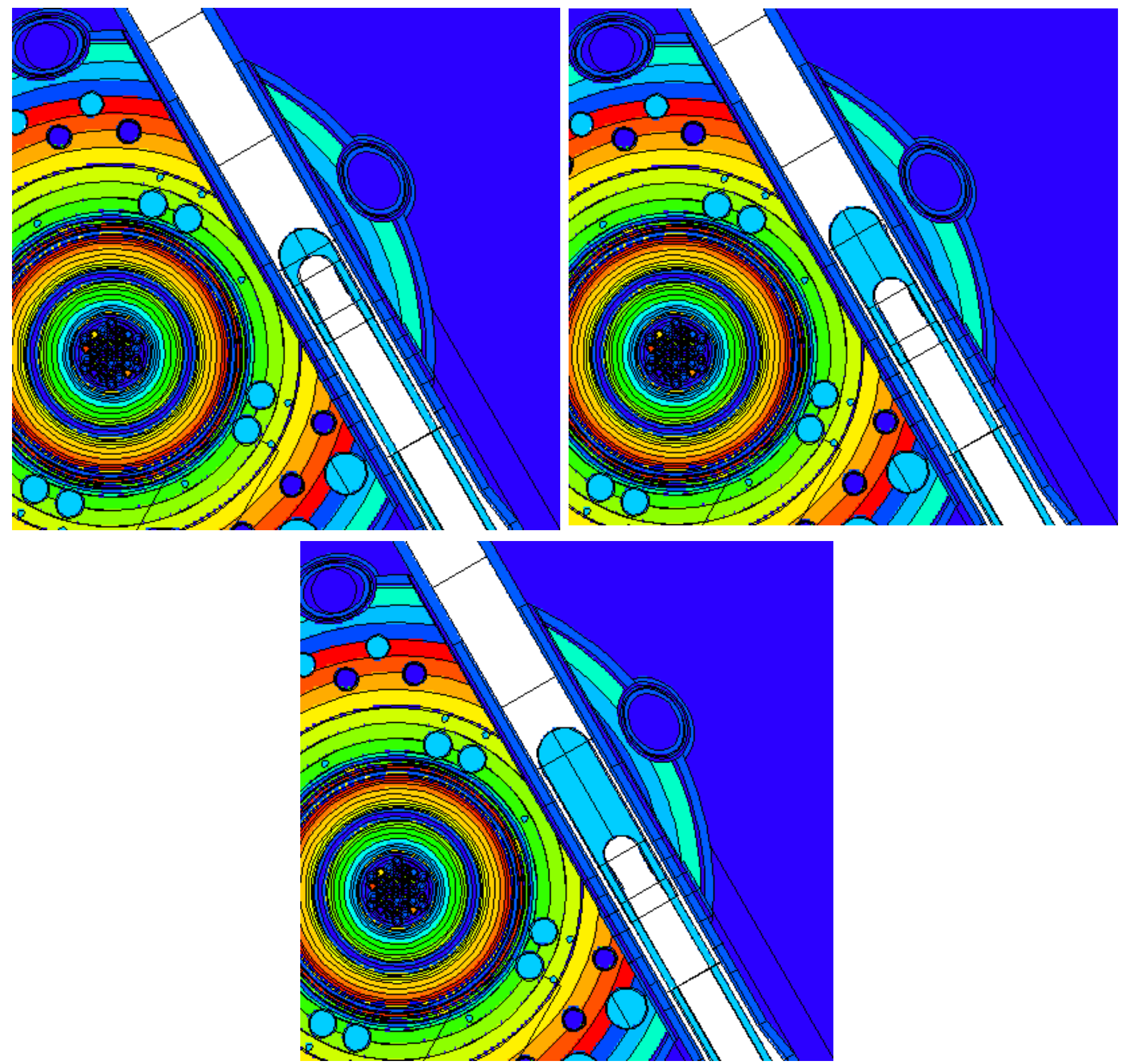

Figure 3.4.1. Cold source configurations of existing design but placed in a through tube (connected HB-1 and HB-4 tube) and centered with regard to reactor core. CS length is increased from the present configuration. 
HB4, 0.13-5.1 meV, $25.1-4.0 \AA$

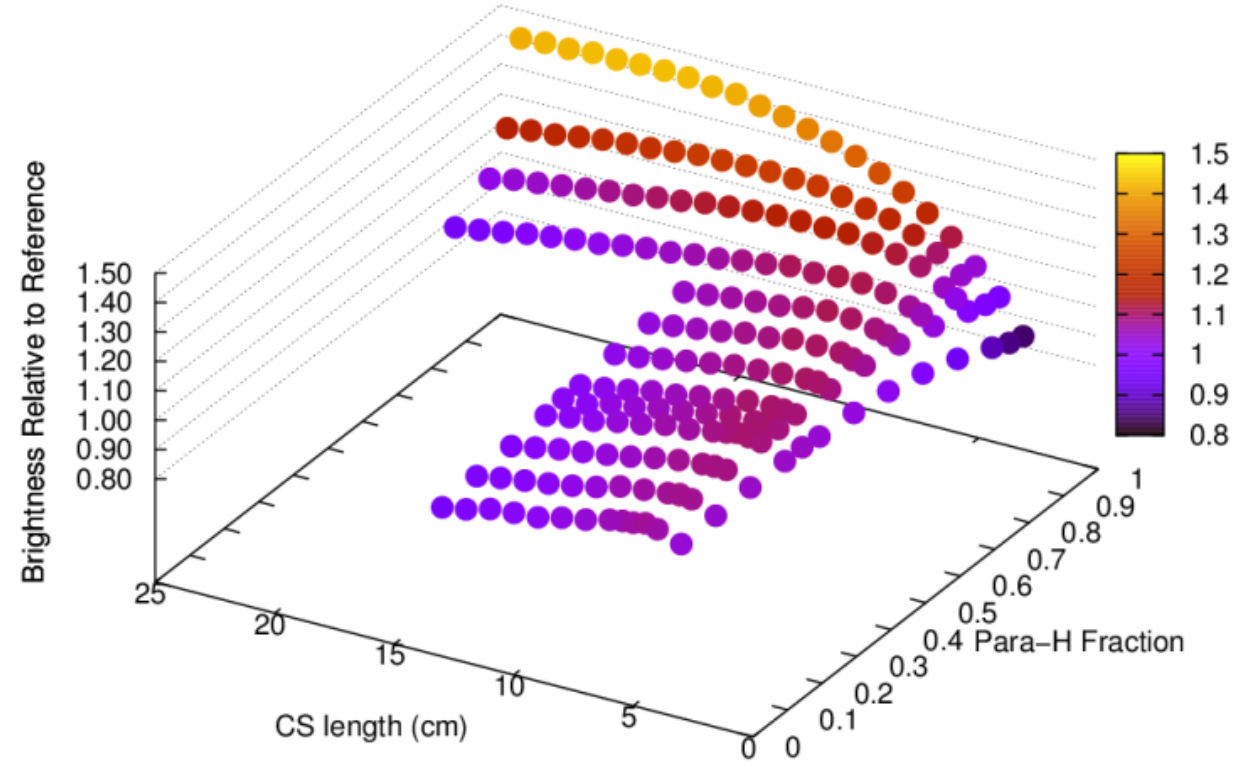

Figure 3.4.2. CS brightness for through tube arrangement, in the 0.13-5.1 meV range (25.1 - 4.0 $\AA$ ), as a function of CS length and para-hydrogen fraction.

HB4, $0.13-5.1 \mathrm{meV}, 25.1-4.0 \AA$

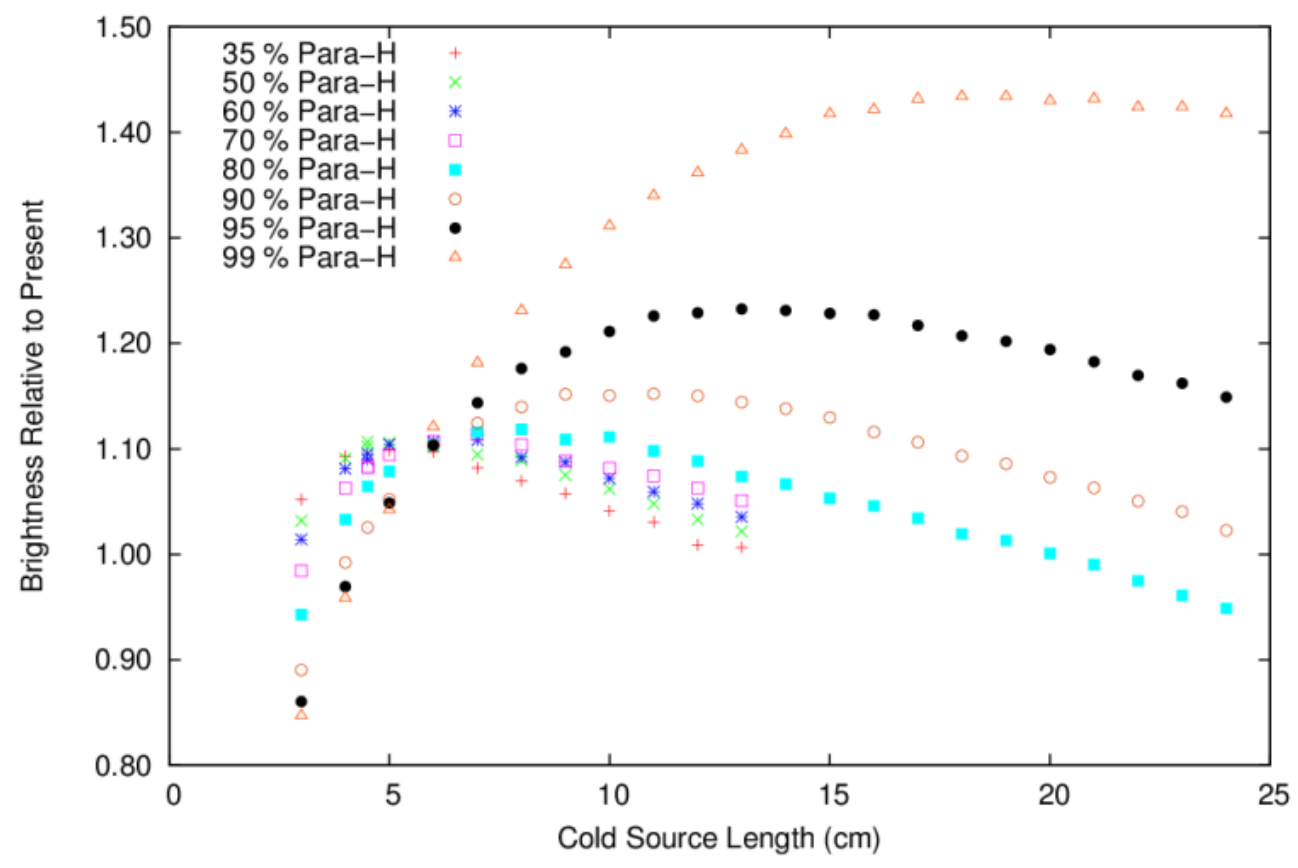

Figure 3.4.3. Brightness of the CS in through tube arrangement, as viewed from the HB-4 side, in the 0.13-5.1 meV range (25.1 - 4.0 $\AA$ ), as a function of CS length for various para-hydrogen fractions. 


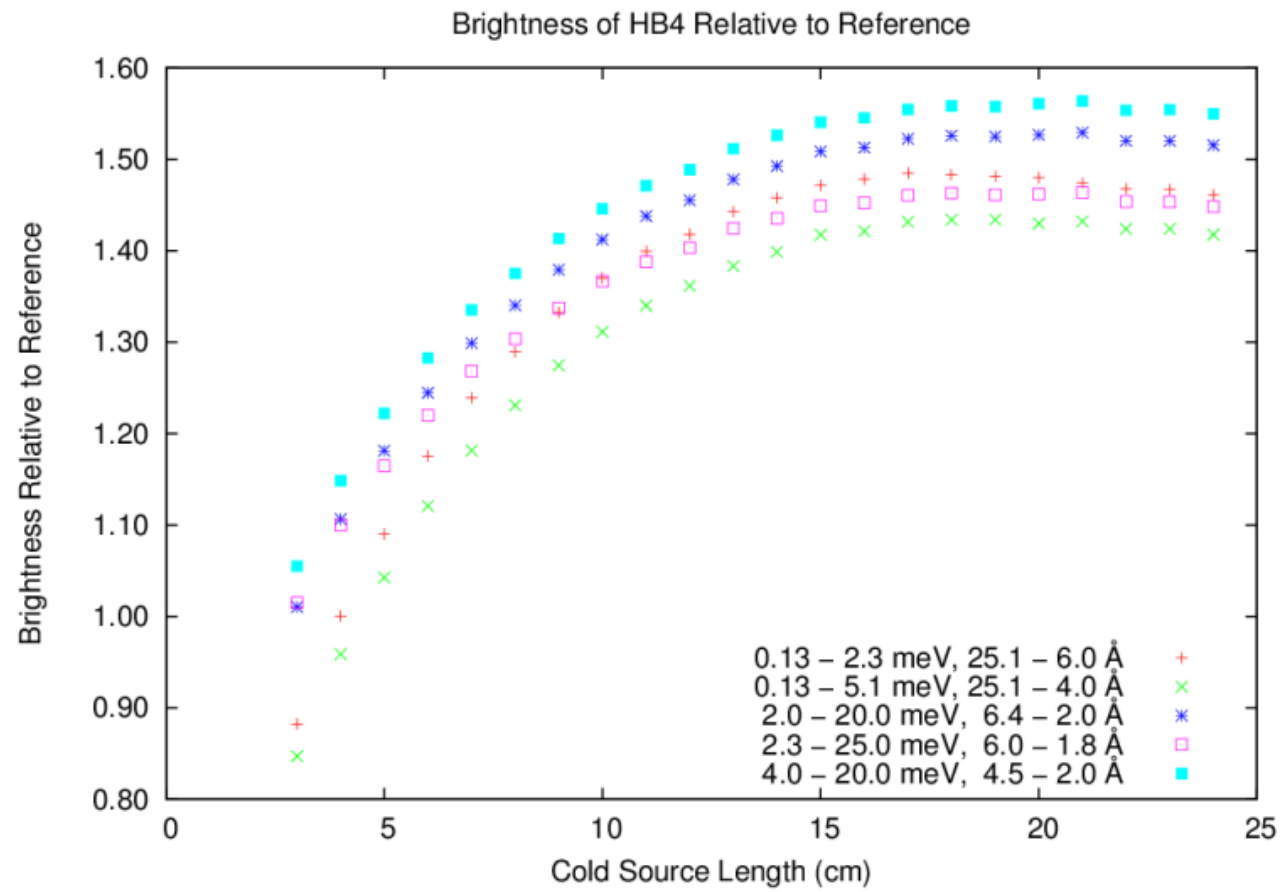

Figure 3.4.4. Brightness of the CS in through tube arrangement, viewed from the HB-4 side versus CS length for $99 \%$ para-hydrogen fraction.

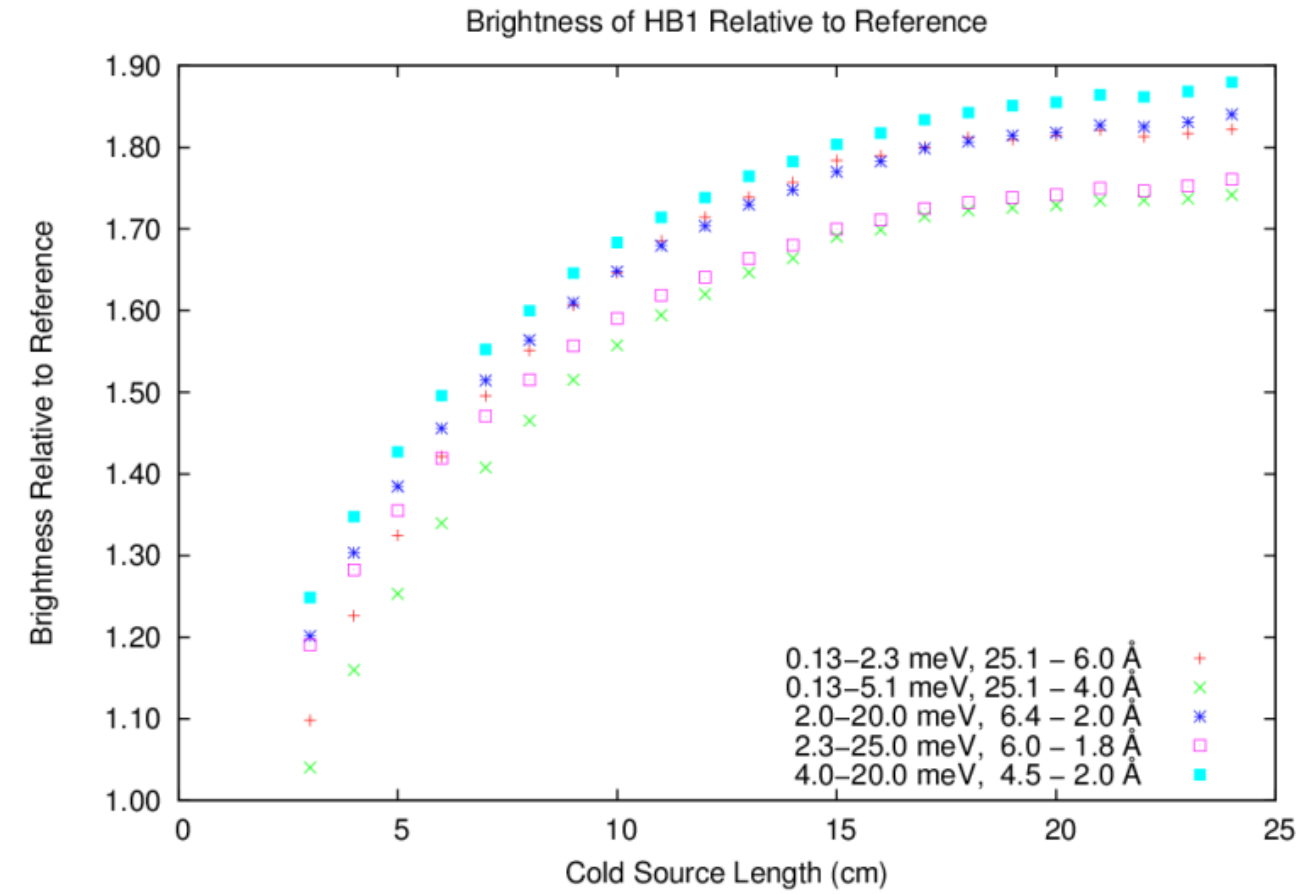

Figure 3.4.5. Brightness of the CS in through tube arrangement, viewed from the HB-1 side versus CS length for the $99 \%$ para-hydrogen fraction. 


\subsection{HB-1 AND HB-4 CONNECTED, COLD SOURCE SHAPE MODIFIED, AND LENGTH EXTENDED}

In the cold source configurations considered so far, the CS vessel shape was modified only by extending the cylindrical part of the CS capsule and by increasing the radius, while the shapes of the CS viewed surfaces were the same as in the existing CS, namely the spherical dome facing the HB-1 side and the reentrant concave dome facing HB-4. In this section, the CS shape was changed by replacing the curved viewed surfaces with flat (plane) surfaces. When the viewed surfaces (the planes) are perpendicular to the beamline direction the CS capsule has a simple cylindrical shape, as shown in Fig. 3.5.1, in the top left panel. The distance between the viewed surfaces or the "thickness" T of the CS is, in this case, the same as the length (L) of the CS in the beam tube direction. When the viewed surfaces are rotated by an angle (Theta) with respect to the axis at the center of the cold source, perpendicular to the horizontal plane through the beam tube center, the shape of the CS changes as shown in Fig. 3.5.1. For a fixed thickness $\mathrm{T}$ of the $\mathrm{CS}$, the length $\mathrm{L}$ varies as $\mathrm{T} / \mathrm{cos}$ (Theta) and the volume increases as $\left(\mathrm{V}_{0} / \mathrm{T}\right) \mathrm{L}$, where $\mathrm{V}_{0}$ is the volume at Theta equal to 0 degrees.

The parameters varied in this series of calculations were the CS thicknesses (T), inclination angle Theta, and para-hydrogen fraction. Based on the results presented in section 3.4, the calculations were centered on high para-hydrogen fractions and larger CS thicknesses.

The results showed that the CS brightness depends mostly on the CS length L, and the para hydrogen fraction. Some of the results are shown in Fig. 3.5.2, which displays CS brightness as a function of para-hydrogen fraction, SC length (L), and inclination angle (Theta). Again, as it was observed in section 3.4, the optimal CS length varies with para hydrogen fraction and the highest brightness was obtained at the highest para-hydrogen fraction.

Fig. 3.5.3 shows brightness of the CS viewed from HB-4 side, in the 0.13-5.1 meV range (25.1 $4.0 \AA$ ), as a function of CS length (L), for the para-hydrogen fraction 99\%. Fig. 3.5.4 gives the same plot for the CS viewed from the HB-1 side. The curves for different CS thicknesses and angles of inclinations show very similar shape and to a large extent fall along the same curve. The peak brightness is achieved for the CS length in the viewed direction in the range $\sim 18-22$ $\mathrm{cm}$. Just about the same peak brightness can be obtained with various combinations of CS thicknesses and angles of inclination, when they combine to yield the CS length $\sim 20 \mathrm{~cm}$, which indicates that the CS length (which is proportional to the volume) is the parameter dictating the CS characteristics.

Fig. 3.5.5 shows the brightness of the CS from HB-4 side, and Fig. 3.5.6 from the HB-1 side, for several energy ranges, as a function of the CS length in the beamline direction, for CS thicknesses $8 \mathrm{~cm}$ and para-hydrogen content is 99\%. The optimal CS length is in the range 19 $23 \mathrm{~cm}$ (inclination angle Theta 65 to 70 degrees). There is a slight difference in optimal length $\mathrm{L}$ between the HB-4 and HB-1 side and the HB-4 side shows a slight dependence of optimal length on neutron energy interval. This configuration gives of one of the highest brightnesses in this series of calculations. The brightness increase for this configuration was in the range from $68 \%$ to $88 \%$ and was about the same on the HB-4 and HB-1 side (see Figs. 3.5.5 and 3.5.6). 
The benefit related to inclined viewed surfaces as opposed to the surfaces perpendicular to the beamline direction appears to be rather small, if any at all, as illustrated in Table 3.5.1. Table 3.5.1 compares the brightness of two configurations with equal volume (and length in the beamline direction). Configuration A has inclined viewed surfaces and Configuration B has viewed surfaces perpendicular to the beamline. While both configurations have brightness significantly higher than the reference CS, the configuration with inclined viewed surfaces shows brightness up to $\sim 10 \%$ higher than the configuration with viewed surfaces perpendicular to the beamline direction.

For the CS thickness close to the optimal CS length of $\sim 20 \mathrm{~cm}$, the inclined surfaces do not offer practically any advantage. This is illustrated in Table 3.5.3, which compares CS brightness for the CS thickness $19 \mathrm{~cm}$, and inclination angles Theta from 0 to 40 degrees. 

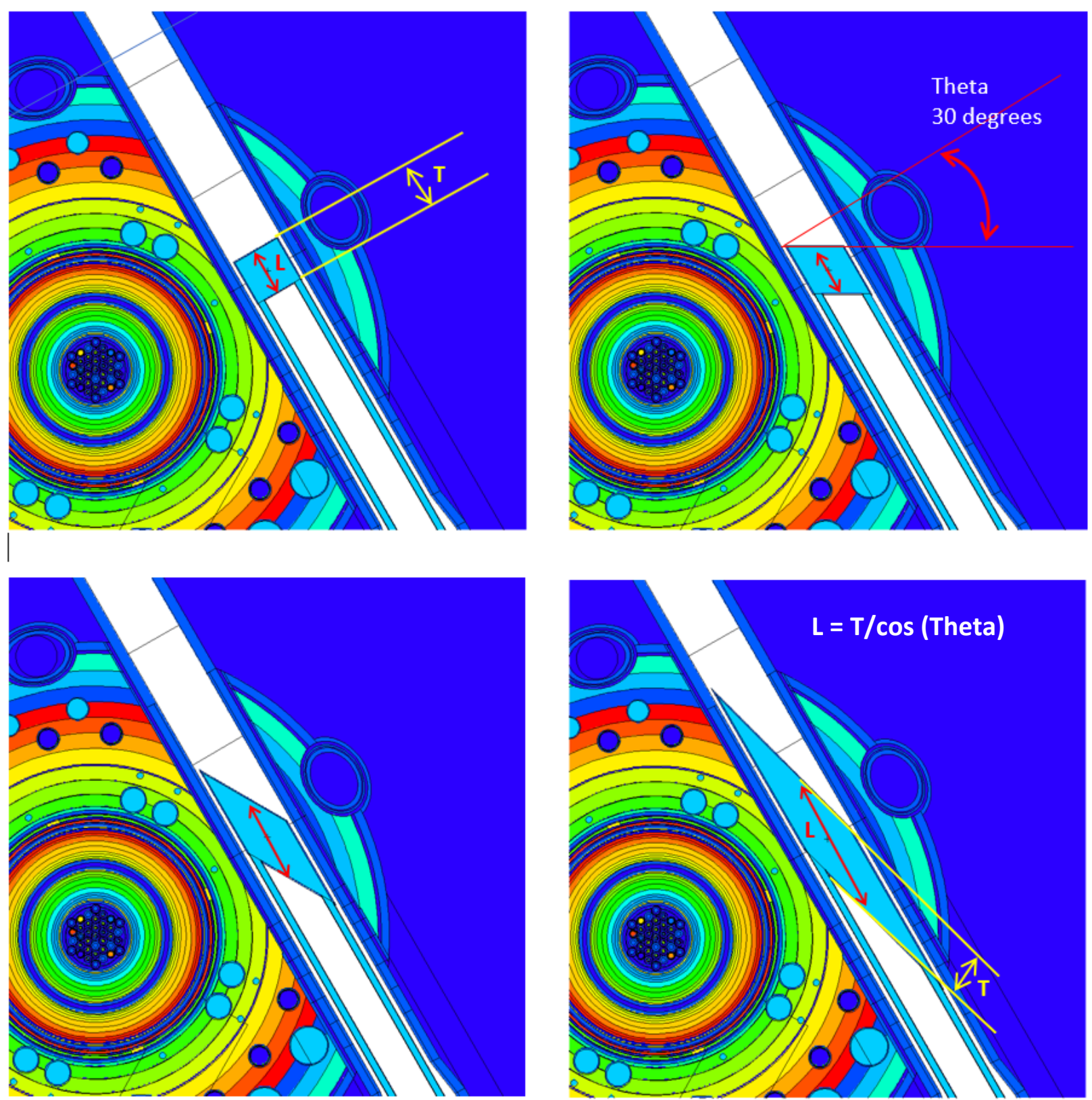

Figure 3.5.1. Examples of the CS configurations modified in shape. From top left in the clockwise direction: for the "inclination" angle Theta $=0$ degrees, 30 degrees, 60 degrees, and 75 degrees. The yellow arrow marks the "thickness" (T) of the CS, which is $9 \mathrm{~cm}$ for all the cases shown. The red arrow indicates the length (L) of the $\mathrm{CS}$ in the viewed direction. 
$\mathrm{HB} 4,0.13-5.1 \mathrm{meV}, 25.1-4.0 \AA$

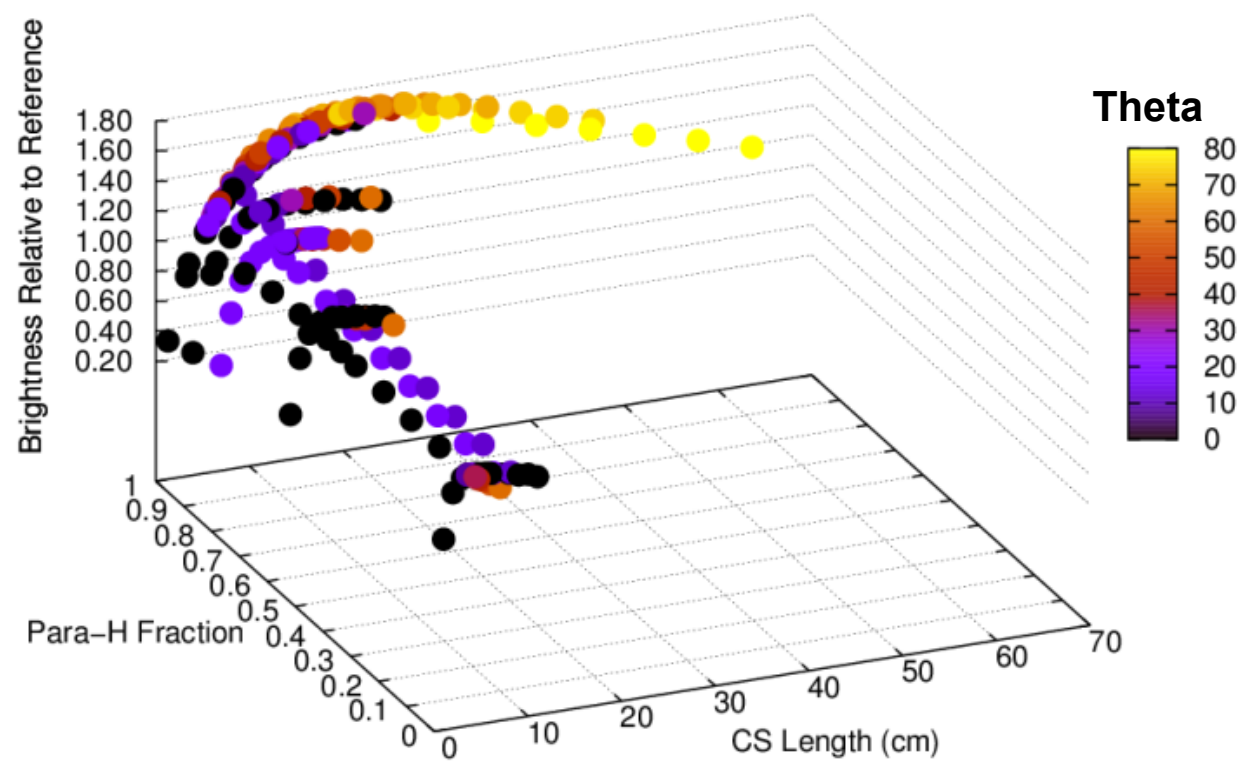

Figure 3.5.2. Brightness of the shape modified CS viewed from HB-4 side, in the 0.13-5.1 meV range (25.1 4.0 A), as a function of para-hydrogen fraction and CS length $(L)$ in the beam tube direction. The color of the points indicates the inclination angle Theta as shown in the color scale on the right side. The brightness is given relative to the brightness of the reference $C S$. 
HB4, 0.13-5.1 meV, $25.1-4.0 \AA$

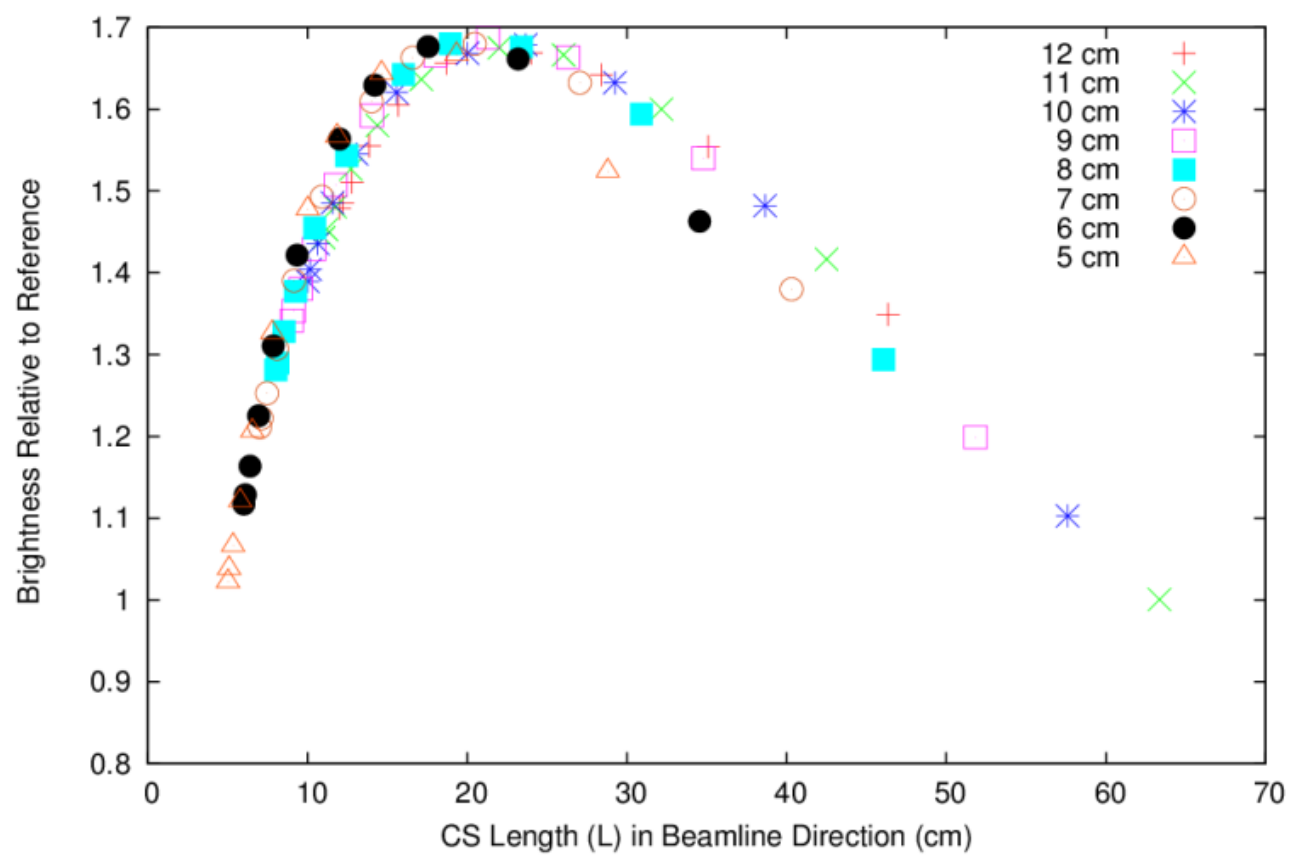

Figure 3.5.3. Brightness of the shape modified CS viewed from HB-4 side, in the 0.13-5.1 meV range (25.1 4.0 $\AA$ ), as a function of CS length (L) for various CS thicknesses (T); para-hydrogen fraction is $99 \%$.

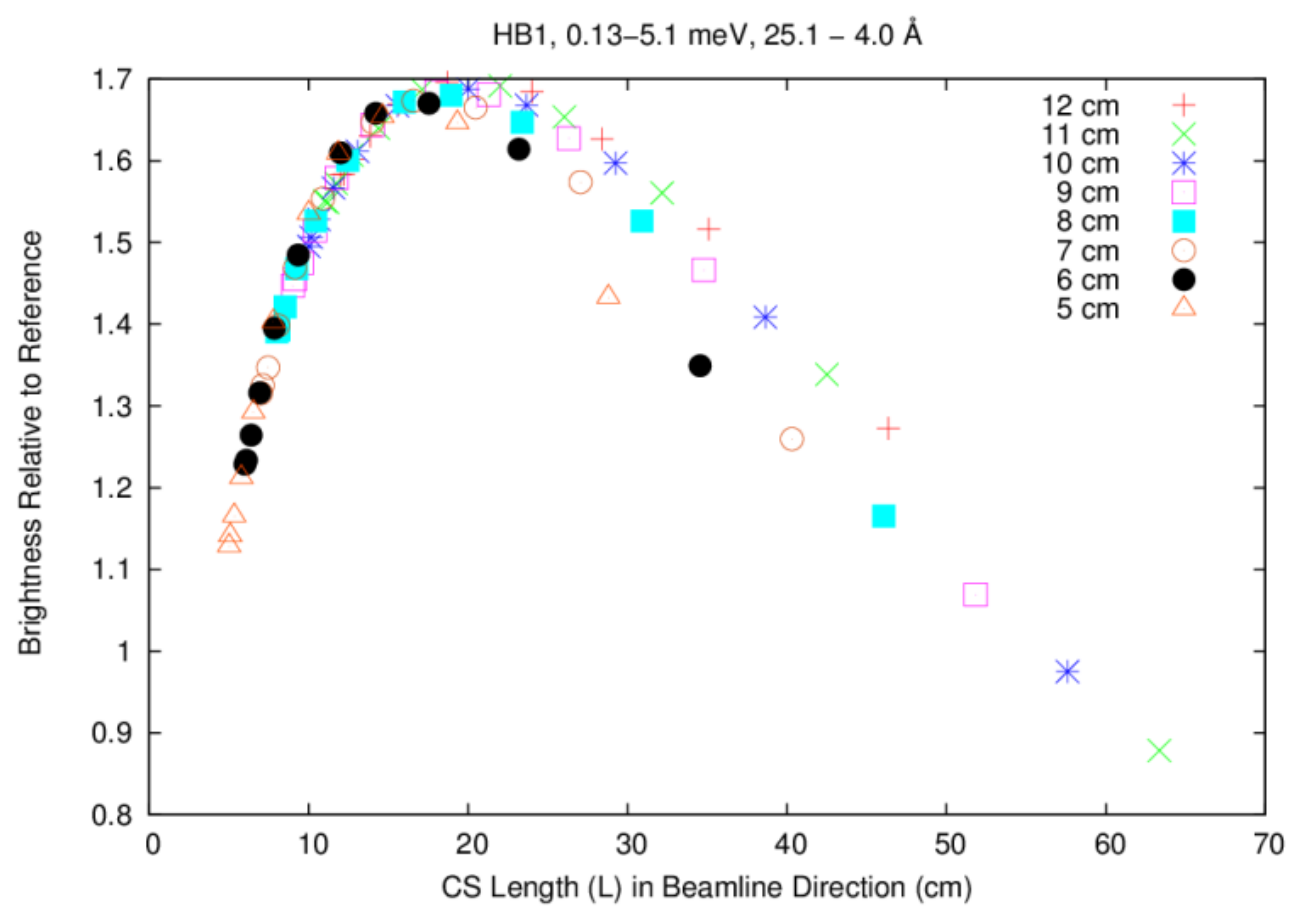

Figure 3.5.4. Brightness of the shape modified CS viewed from HB-1 side, in the 0.13-5.1 meV range (25.1 4.0 $\AA$ ), as a function of CS length (L) for various CS thicknesses (T); para-hydrogen fraction is $99 \%$. 
HB4, CS Thickness $8 \mathrm{~cm}$

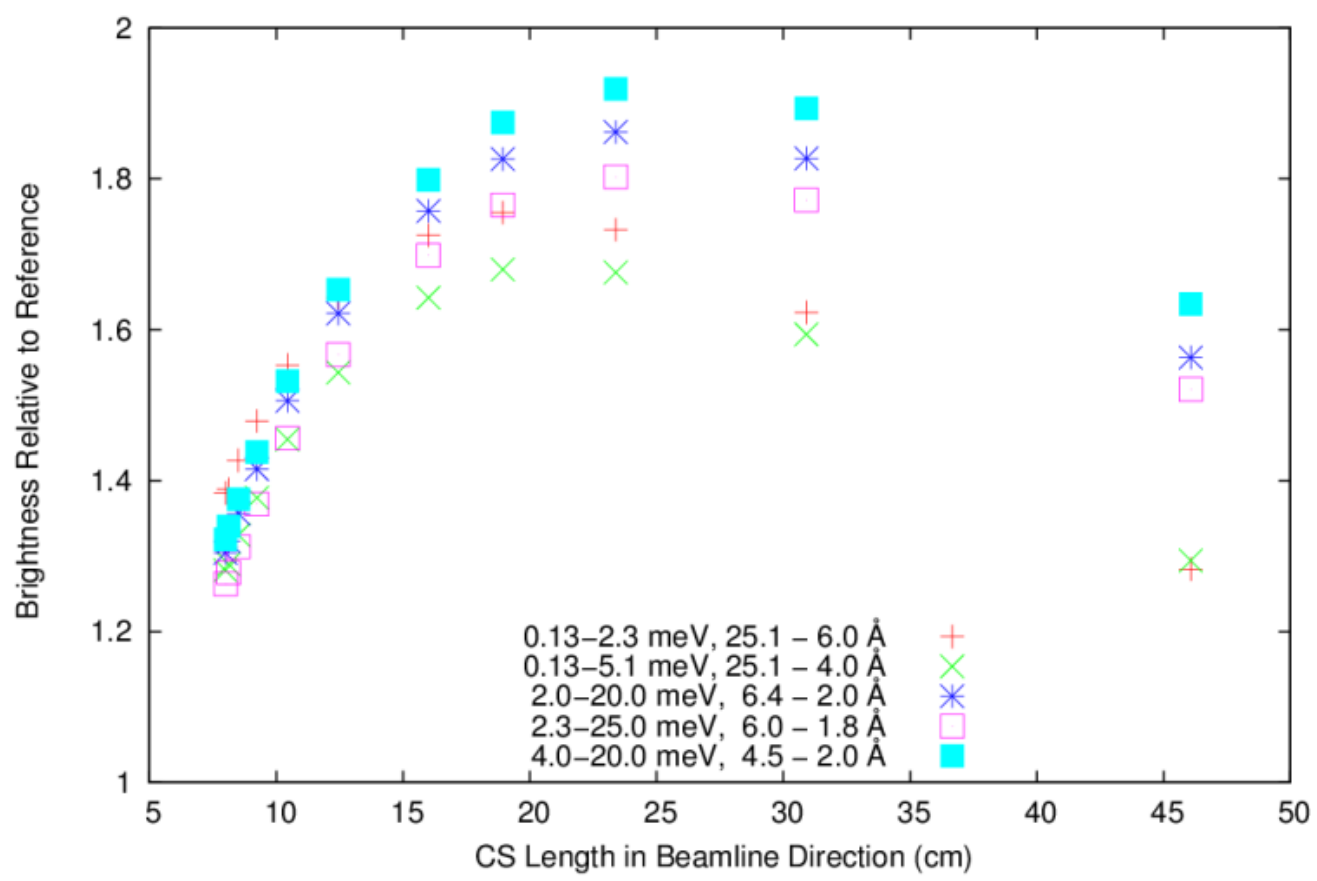

Figure 3.5.5. Brightness of the shape modified CS viewed from HB-4 side, as a function of CS length (L) in the beamline direction, for CS thickness (T) $8 \mathrm{~cm}$; for selected energy ranges; para-hydrogen fraction is $99 \%$.

HB1, CS Thickness $8 \mathrm{~cm}$

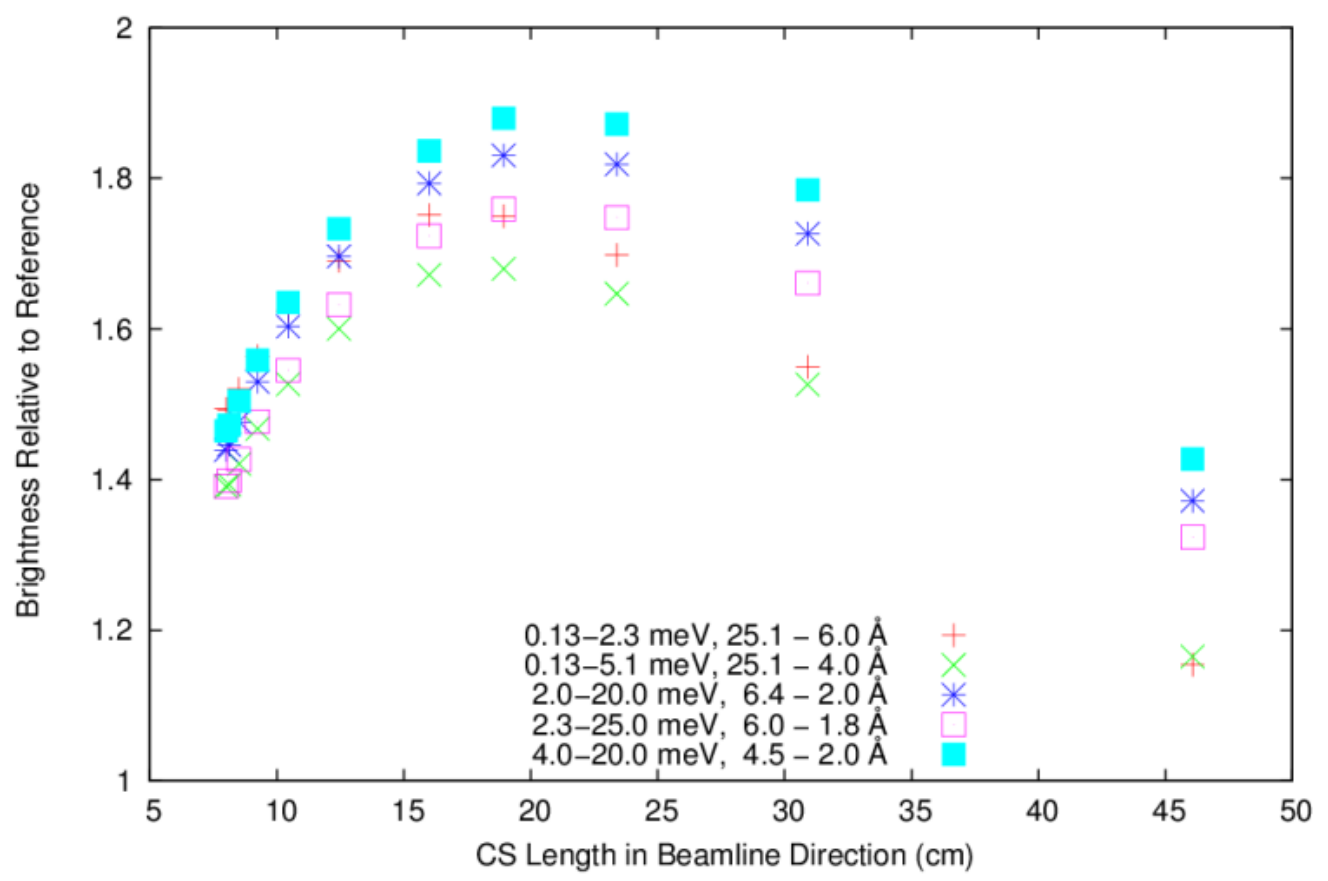

Figure 3.5.6. Brightness of the shape modified CS viewed from HB-1 side, as a function of CS length (L) in the beamline direction, for CS thickness (T) $8 \mathrm{~cm}$; for selected energy ranges; para-hydrogen fraction is $99 \%$. 
Table 3.5.1. Comparison of the CS brightness for the CS with inclined surfaces (Configuration A), and surfaces perpendicular to the beamline direction (Configuration $B$ ).

\begin{tabular}{|c|c|c|c|c|c|}
\hline \multirow[b]{2}{*}{$\begin{array}{l}\text { Cold source } \\
\text { configuration }\end{array}$} & \multicolumn{5}{|c|}{ Cold Source Brightness } \\
\hline & $\begin{array}{c}0.13-2.3 \mathrm{meV}, \\
25.1-6 \AA \\
\end{array}$ & $\begin{array}{c}0.13-5.1 \mathrm{meV} \\
25.1-4 \AA \\
\end{array}$ & $\begin{array}{c}2.0-20.0 \mathrm{meV} \\
6.4-2.0 \AA \\
\end{array}$ & $\begin{array}{c}2.3-25.0 \mathrm{meV} \\
6.0-1.8 \AA \\
\end{array}$ & $\begin{array}{c}4.0-20.0 \mathrm{meV} \\
4.5-2.0 \AA \\
\end{array}$ \\
\hline \multicolumn{6}{|l|}{ Configuration $\mathrm{A}$ : } \\
\hline $\mathrm{T}=8 \mathrm{~cm}$ & $1.76^{\mathrm{a}}$ & 1.68 & 1.83 & 1.77 & 1.87 \\
\hline $\begin{array}{c}\text { Theta } 65 \mathrm{deg} . \\
\mathrm{L}=18.9 \mathrm{~cm}\end{array}$ & $1.75^{b}$ & 1.68 & 1.83 & 1.76 & 1.88 \\
\hline \multicolumn{6}{|l|}{ Configuration B } \\
\hline $\mathrm{T}=19 \mathrm{~cm}$, & $1.70^{\mathrm{a}}$ & 1.61 & 1.64 & 1.56 & 1.66 \\
\hline $\begin{array}{l}\text { Theta } 0 \text {, } \\
\mathrm{L}=19 \mathrm{~cm}\end{array}$ & $1.80^{\mathrm{b}}$ & 1.71 & 1.76 & 1.68 & 1.79 \\
\hline \multirow{2}{*}{ Ratio B/A } & $0.97^{\mathrm{c}}$ & 0.96 & 0.90 & 0.88 & 0.89 \\
\hline & $1.03^{\mathrm{d}}$ & 1.02 & 0.96 & 0.95 & 0.95 \\
\hline
\end{tabular}

${ }^{a}$ Brightness of the HB-4 side relative to the reference CS HB-4 side.

${ }^{b}$ Brightness of the HB-1 side relative to the reference CS HB-4 side.

${ }^{\mathrm{c}}$ Ratio of the brightness (Configuration B / Configuration A) for the HB-4 side.

${ }^{\mathrm{d}}$ Ratio of the brightness (Configuration B / Configuration A) for the HB-1.

Table 3.5.2. CS brightness for the CS thickness $19 \mathrm{~cm}, 99 \%$ para-hydrogen fraction and different inclination angles Theta. Values are given relative to the value for THETA $=0$.

\begin{tabular}{cccccc}
\hline $\begin{array}{c}\text { Inclination Angle } \\
\text { Theta } \\
\text { (degrees) }\end{array}$ & $\begin{array}{c}\text { Cold Source Brightness } \\
0.13-2.3 \mathrm{meV}, \\
25.1-6 \AA\end{array}$ & $\begin{array}{c}0.13-5.1 \mathrm{meV} \\
25.1-4 \AA\end{array}$ & $\begin{array}{c}2.0-20.0 \mathrm{meV} \\
6.4-2.0 \AA\end{array}$ & $\begin{array}{c}2.3-25.0 \mathrm{meV} \\
6.0-1.8 \AA\end{array}$ & $\begin{array}{c}4.0-20.0 \mathrm{meV} \\
4.5-2.0 \AA\end{array}$ \\
\hline 0 & $1.00^{\mathrm{a}}$ & 1.00 & 1.00 & 1.00 & 1.00 \\
& $1.00^{\mathrm{b}}$ & 1.00 & 1.00 & 1.00 & 1.00 \\
& $1.00^{\mathrm{a}}$ & 1.01 & 1.01 & 1.01 & 1.01 \\
10 & $1.00^{\mathrm{b}}$ & 1.00 & 1.00 & 1.00 & 1.00 \\
& $1.01^{\mathrm{a}}$ & 1.01 & 1.02 & 1.02 & 1.02 \\
& $1.00^{\mathrm{b}}$ & 1.00 & 1.00 & 1.00 & 1.00 \\
& $1.01^{\mathrm{a}}$ & 1.02 & 1.04 & 1.04 & 1.04 \\
& $1.00^{\mathrm{b}}$ & 1.00 & 1.01 & 1.01 & 1.02 \\
& & & & & 1.00 \\
& $1.01^{\mathrm{a}}$ & 1.02 & 1.05 & 1.05 & 1.06 \\
& $0.98^{\mathrm{b}}$ & 0.99 & 1.01 & 1.01 & 1.02 \\
\hline
\end{tabular}

${ }^{a}$ Brightness of the HB-4 side normalized to the values at Theta $=0$ degrees.

${ }^{b}$ Brightness of the HB-1 side normalized to the values at Theta $=0$ degrees. 


\subsection{COMPARISON OF DETAILED CS BRIGHTNESS VERSUS ENERGY VARIATIONS}

The cold source configurations were so far compared based on the brightness averaged over the selected energy ranges. Here a detailed dependence of the CS brightness on the neutron energy will be presented for one CS selected from each of the CS modifications considered in the section 3.1 to 3.5. Each CS configuration was selected so that it provides the highest brightness or within a few percent of the highest brightness in each series of modifications considered, while giving preference to the configurations with lower CS heating rates. All calculations assumed 99\% para-H fraction. Table 3.6.1 summarizes the selected configurations, and gives the brightness relative to the reference CS brightness. The CS heating is also given relative to the reference cold source heating (which is $2194 \mathrm{~W}$ at the reactor power $85 \mathrm{MW}$ ).

Table 3.6.1. Comparison of brightness and heating for selected cold source configurations. All values are given relative to the reference $\mathrm{CS}$ brightness and heating.

\begin{tabular}{|c|c|c|c|c|c|c|}
\hline \multirow{2}{*}{$\begin{array}{l}\text { Cold Source } \\
\text { Configuration } \\
\text { (Described in } \\
\text { Section) }\end{array}$} & \multicolumn{5}{|c|}{$\begin{array}{l}\text { Cold Source Brightness Relative to the Reference Cold Source } \\
\text { In the Energy/Wavelength Interval }\end{array}$} & \multirow{2}{*}{$\begin{array}{l}\text { Heating } \\
\text { Factor }\end{array}$} \\
\hline & $\begin{array}{l}0.13-2.3 \mathrm{meV} \\
25.1-6 \AA\end{array}$ & $\begin{array}{l}0.13-5.1 \mathrm{meV} \\
25.1-4 \AA\end{array}$ & $\begin{array}{l}2.0-20.0 \mathrm{meV} \\
6.4-2.0 \AA\end{array}$ & $\begin{array}{l}2.3-25.0 \mathrm{meV} \\
6.0-1.8 \AA\end{array}$ & $\begin{array}{l}4.0-20.0 \mathrm{meV} \\
4.5-2.0 \AA\end{array}$ & \\
\hline $\begin{array}{l}\text { Extended out, } \\
\mathrm{L} 10 \mathrm{~cm} \\
(\text { Section } 3.1)\end{array}$ & 1.20 & 1.16 & 1.31 & 1.26 & 1.36 & 1.20 \\
\hline $\begin{array}{l}\text { Extended in, } \\
\text { L } 15 \mathrm{~cm} \\
\text { (Section } 3.2)\end{array}$ & 1.48 & 1.44 & 1.57 & 1.50 & 1.61 & 1.88 \\
\hline $\begin{array}{l}\text { Extended in, } \\
\text { L } 17 \mathrm{~cm}, \mathrm{R} \text { inc., } \\
\text { (Section } 3.3 \text { ) }\end{array}$ & 1.42 & 1.37 & 1.44 & 1.37 & 1.46 & 2.71 \\
\hline $\begin{array}{l}\text { Through tube, } \\
\text { Extended CS, } \\
\text { L } 18 \mathrm{~cm}, \\
\text { (Section } 3.4 \text { ) }\end{array}$ & $\begin{array}{l}1.47^{\mathrm{a}} \\
1.82\end{array}$ & $\begin{array}{l}1.42 \\
1.74\end{array}$ & $\begin{array}{l}1.52 \\
1.83\end{array}$ & $\begin{array}{l}1.45 \\
1.75\end{array}$ & $\begin{array}{l}1.55 \\
1.87\end{array}$ & 2.20 \\
\hline $\begin{array}{l}\text { Through tube, } \\
\text { Modified CS, } \\
\text { T } 8 \mathrm{~cm} \text {, Theta } 65 \text {, } \\
\text { (Section } 3.5 \text { ) }\end{array}$ & $\begin{array}{c}1.76^{\mathrm{a}} \\
1.75\end{array}$ & $\begin{array}{l}1.68 \\
1.68\end{array}$ & $\begin{array}{l}1.83 \\
1.83\end{array}$ & $\begin{array}{l}1.77 \\
1.76\end{array}$ & $\begin{array}{l}1.87 \\
1.88\end{array}$ & 2.03 \\
\hline
\end{tabular}

"In this configuration HB-1 and HB-4 are connected and form a "through tube". The cold source can be viewed from both the HB-4 and HB-1 side. The numbers on the top are for the brightness on the HB-4 side and the numbers on the bottom are for the HB-1 side. The brightness on the HB-1 side is compared to the reference CS cold brightness on the HB-4 side.

The brightness of the CS (viewed from the HB-4 side) versus energy curves for the reference CS and the five configurations shown in Table 3.6.1 are plotted in Fig. 3.6.1. A more detailed plot of the same brightness curves for the energies below $1 \mathrm{eV}$ is shown in Fig. 3.6.2. To show the changes in brightness more clearly, Fig. 3.6.3 and Fig. 3.6.4 plot the ratio of the CS brightness for the selected configurations to the brightness of the reference CS, for the full energy range and 
for the energies below $1 \mathrm{eV}$, respectively. By increasing the size of the cold source, a significant increase in brightness can be achieved for the wavelengths longer than $\sim 2 \AA$. The increased size works only in combination with operating the CS in a nearly pure para-H state (99\% parahydrogen was assumed in the calculations). It is therefore necessary to use ortho-para converter to maintain hydrogen in a nearly pure para-hydrogen isomeric state.

The brightness increase, relative to the reference CS, varies strongly with the neutron energy. However, the shapes of the curves of the brightness increase versus energy are remarkably similar for all considered configurations. The calculated shapes of the curves of the brightness increase versus energy are also in excellent agreements with the measured changes in brightness as reported by Ooi [15]. The maximum increase in brightness is obtained at around $\sim 35 \AA$, and ranges from $\sim 90 \%$ to $\sim 180 \%$, depending on the CS configuration, as shown in Fig 6.6 .3 and Fig. 6.6.4. Another peak in brightness increase appears at around $3 \AA$ and rages from $\sim 70 \%$ to $~$ $130 \%$. For all but one CS configuration there is a decrease in the brightness in the range $\sim 0.6 \AA$ to $\sim 2 \AA$, with the maximum decrease at $\sim 1.5 \AA$ in the range of $30 \%$ to $50 \%$. The configuration with the through tube and modified CS shape shows the largest brightness increase, and has higher brightness than the reference CS at all wavelengths (see Fig. 3.6.4); however, the shape of the curve of the brightness increase versus energy is similar to the shapes obtained for the other CS configurations.

In the two configurations with connected HB-1 and HB-4 tube the cold source can be viewed from both the HB-1 and the HB-4 side. This by itself offers the possibility of potentially doubling the number of instruments that could be installed on the CS. Fig. 3.6.5 to Fig. 3.6.8 provide the brightness versus energy plots for these two configurations for the HB-4 and HB-1 side of the cold source. Where relative values are given, the brightness of the HB-1 side is given relative to the brightness of the HB-4 side of the reference CS.

On the HB-1 side the brightness increase versus energy shows very similar shape to the shape on HB-4 side, as discussed previously. At wavelengths longer than $\sim 2 \AA$ the brightness increase of the CS with modified shape on the HB-1 side matches closely the brightness increase on the HB4 side (see Fig. 3.6.8). They are both similar to, albeit a little lower, than the brightness increase of the extended CS on the HB-1 side, which has the highest brightness, and reaches $\sim 220 \%$ increase at $\sim 35 \AA$. For the extended CS, the brightness increase on the HB- 1 side is significantly higher, by about $30 \%$ to $90 \%$, than the brightness increase of the HB- 4 side. However, this effect is very minor for the modified CS. Additional calculations revealed that the difference is mostly caused by the different shapes of the viewed surface on the HB-1 and HB-4 side and added aluminum structure on the HB-4 side. The effect of the hydrogen supply lines, which run through the HB-4 side and partially obstruct the view of the CS, is very small.

For the wavelengths shorter than $\sim 1 \AA$, the modified CS has $\sim 40 \%$ higher brightness on the HB4 side and $\sim 10 \%$ higher brightness on the HB- 1 side. The extended CS has $\sim 25 \%$ higher brightness on the HB- 1 side and $\sim 10 \%$ on the HB-4 side. The increase in brightness at short wavelengths means increased background of higher energy neutrons and is not desirable for the instruments using cold neutrons. This may not have a significant impact as all cold neutron beams are extracted through benders or curved guides. 
For the three configurations that keep the HB-4 and HB-1 separated, the impact of the CS modifications on the HB-1 is also of interest. The change in brightness of the HB-1 side is shown in Fig. 3.6.9 and Fig. 3.6.10. When the CS is extended outwards, the change in HB-1 brightness is negligible. When CS is extended inwards, and the HB-1 is retracted into the region with lower neutron flux, the HB-1 brightness decreases by $\sim 25$ to $35 \%$ for the case with the existing CS radius, and by $45 \%$ to $55 \%$ for the CS with increased radius. This severe decrease in HB-1 brightness needs to be taken into account when CS modifications are assessed. As mentioned before it may be possible to keep HB-1 in present position, and this needs to be considered if one of these configurations becomes of interest.

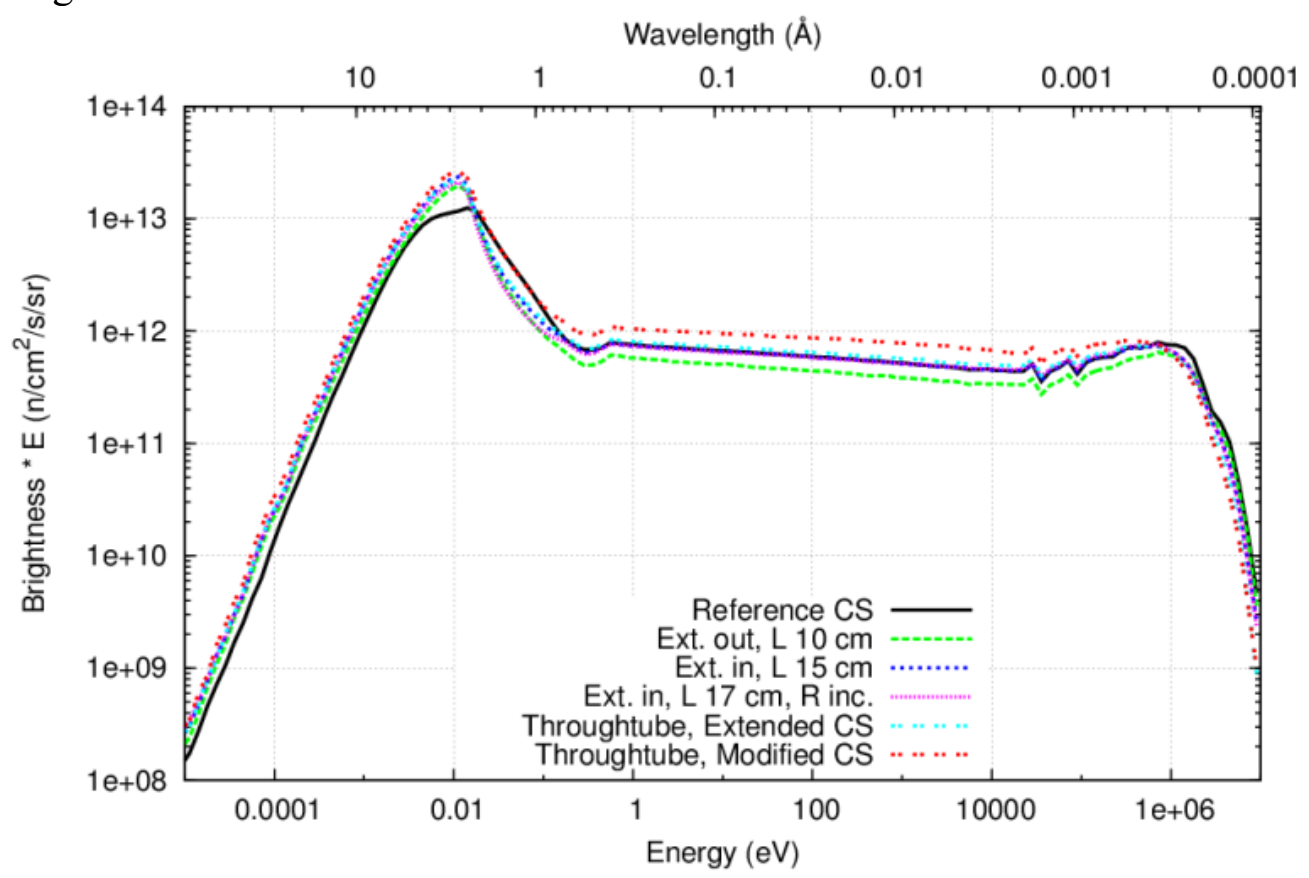

Figure 3.6.1. Cold source brightness versus energy, as viewed from the HB-4 side, for the reference CS and the five configurations shown in Table 3.6.1. 


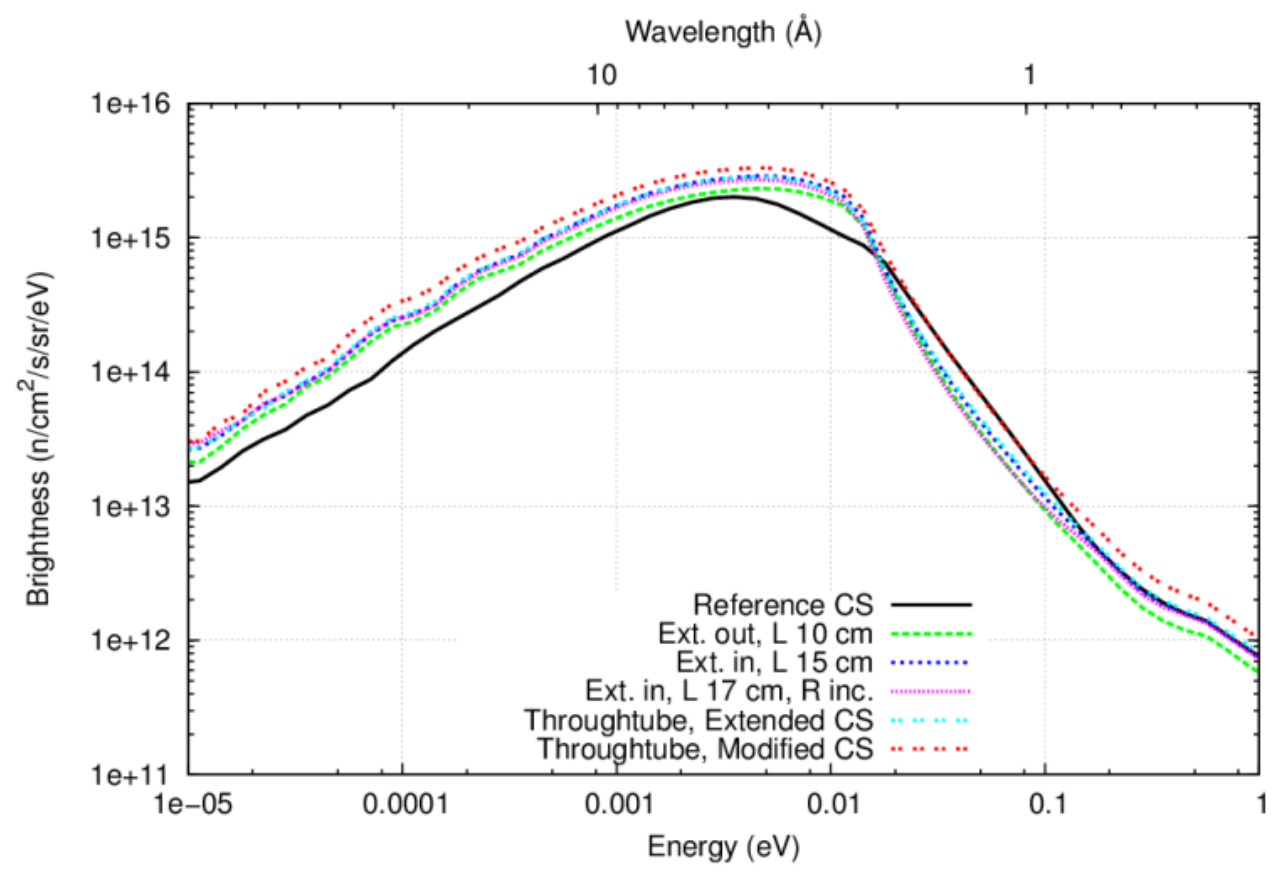

Figure 3.6.2. Cold source brightness versus energy (for energies below $1 \mathrm{eV}$ ), as viewed from the HB-4 side, for the reference $\mathrm{CS}$ and the five selected configurations. 


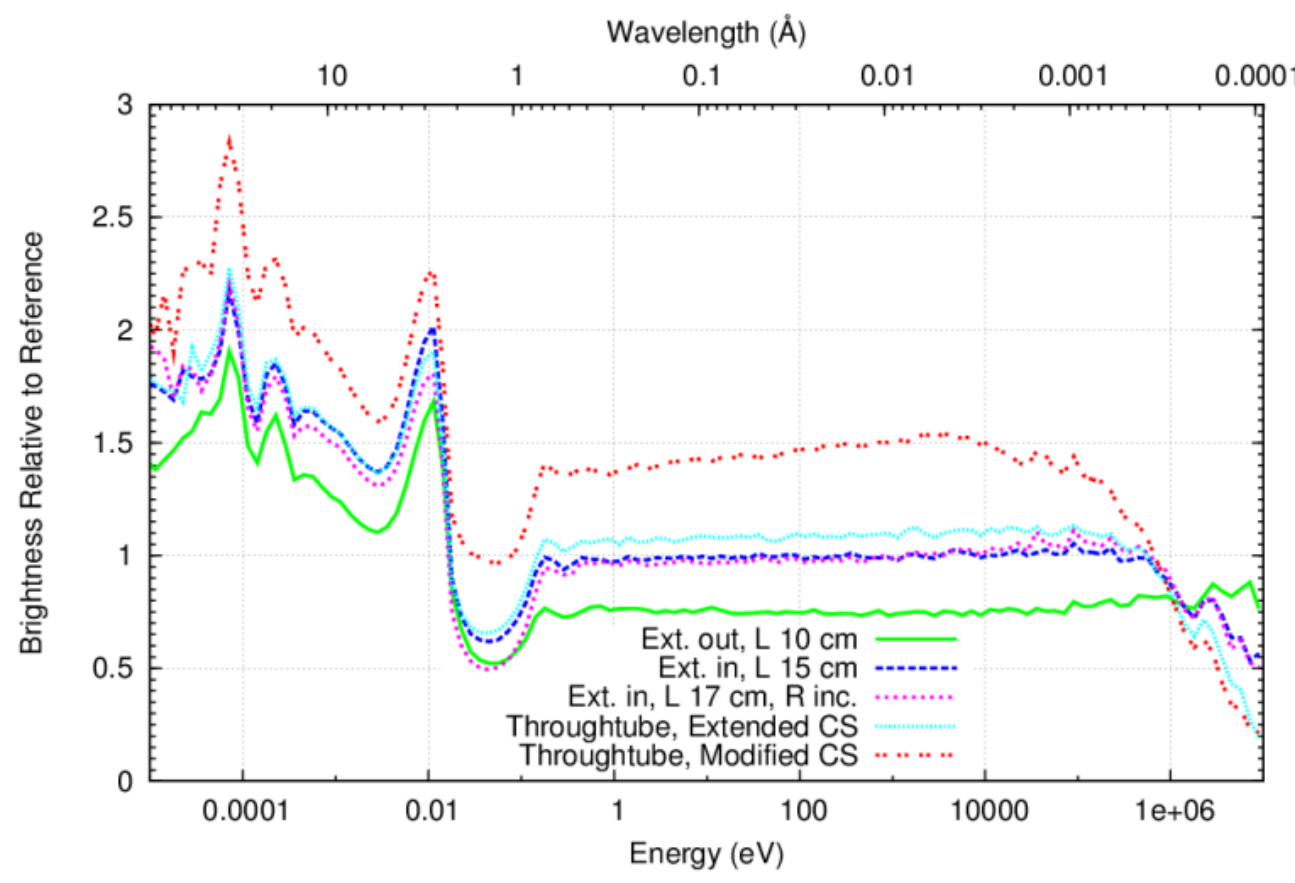

Figure 3.6.3. Cold source brightness versus energy, relative to the reference cold source, as viewed from the HB-4 side, for the five selected configurations.

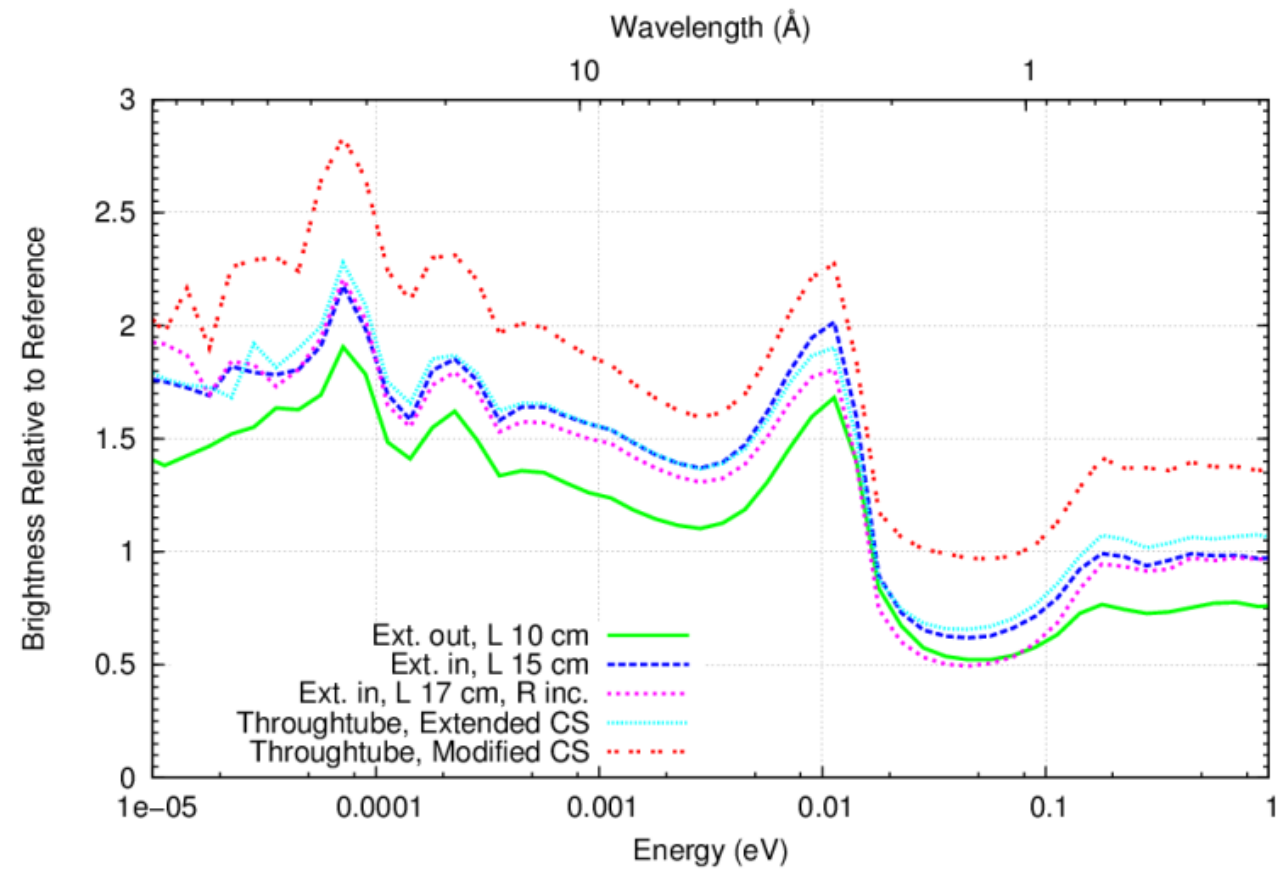

Figure 3.6.4. Cold source brightness versus energy, relative to the reference cold source, as viewed from the HB-4 side, for the five selected configurations, for the energies below $1 \mathrm{eV}$. 


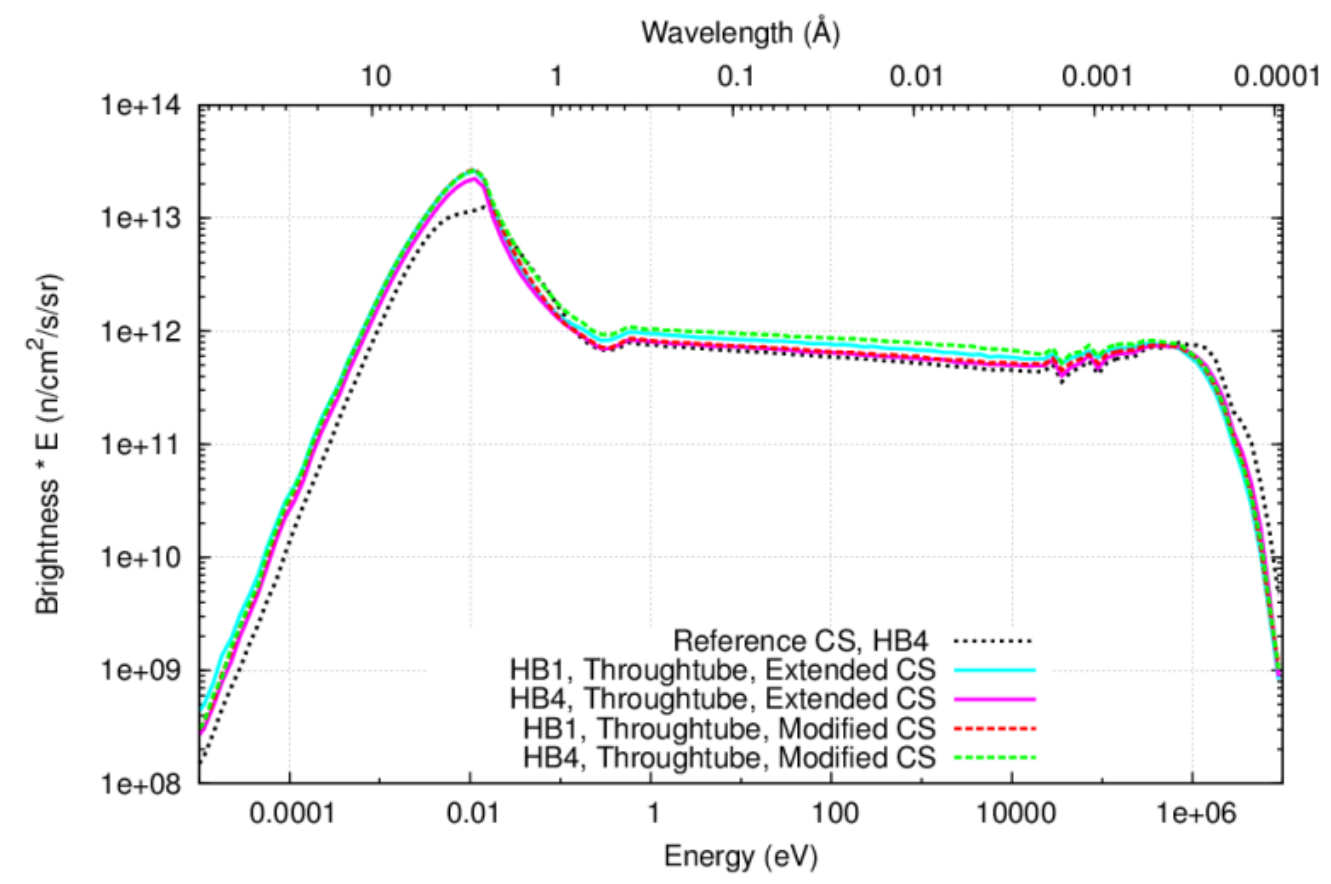

Figure 3.6.5. Cold source brightness versus energy, for the reference CS and the two CS configurations with the through tube that allows viewing of the CS from HB-4 and HB-1 side.

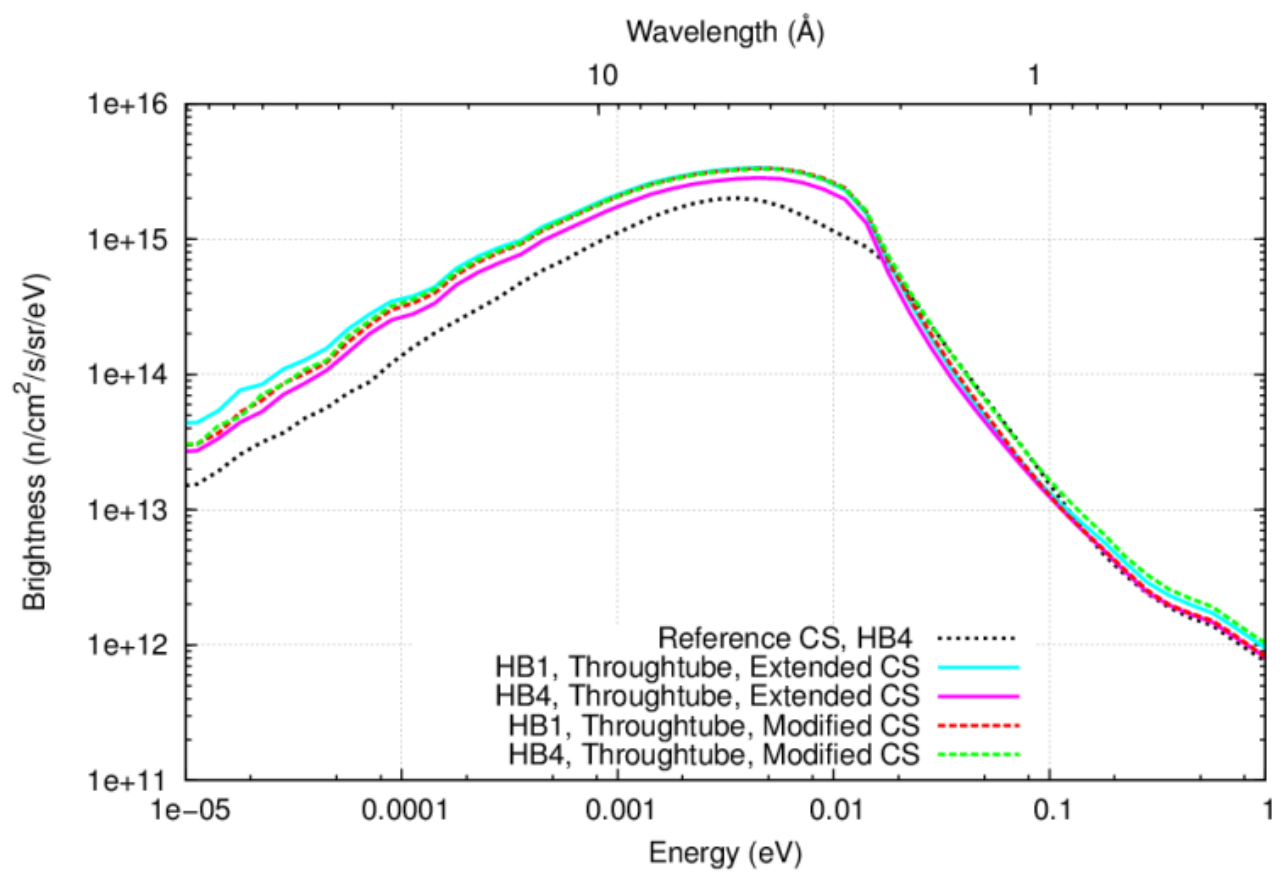

Figure 3.6.6. Cold source brightness versus energy, for energies below $1 \mathrm{eV}$, for the reference $\mathrm{CS}$ and the two CS configurations with the through tube that allows viewing of the CS from HB-4 and HB-1 side. 


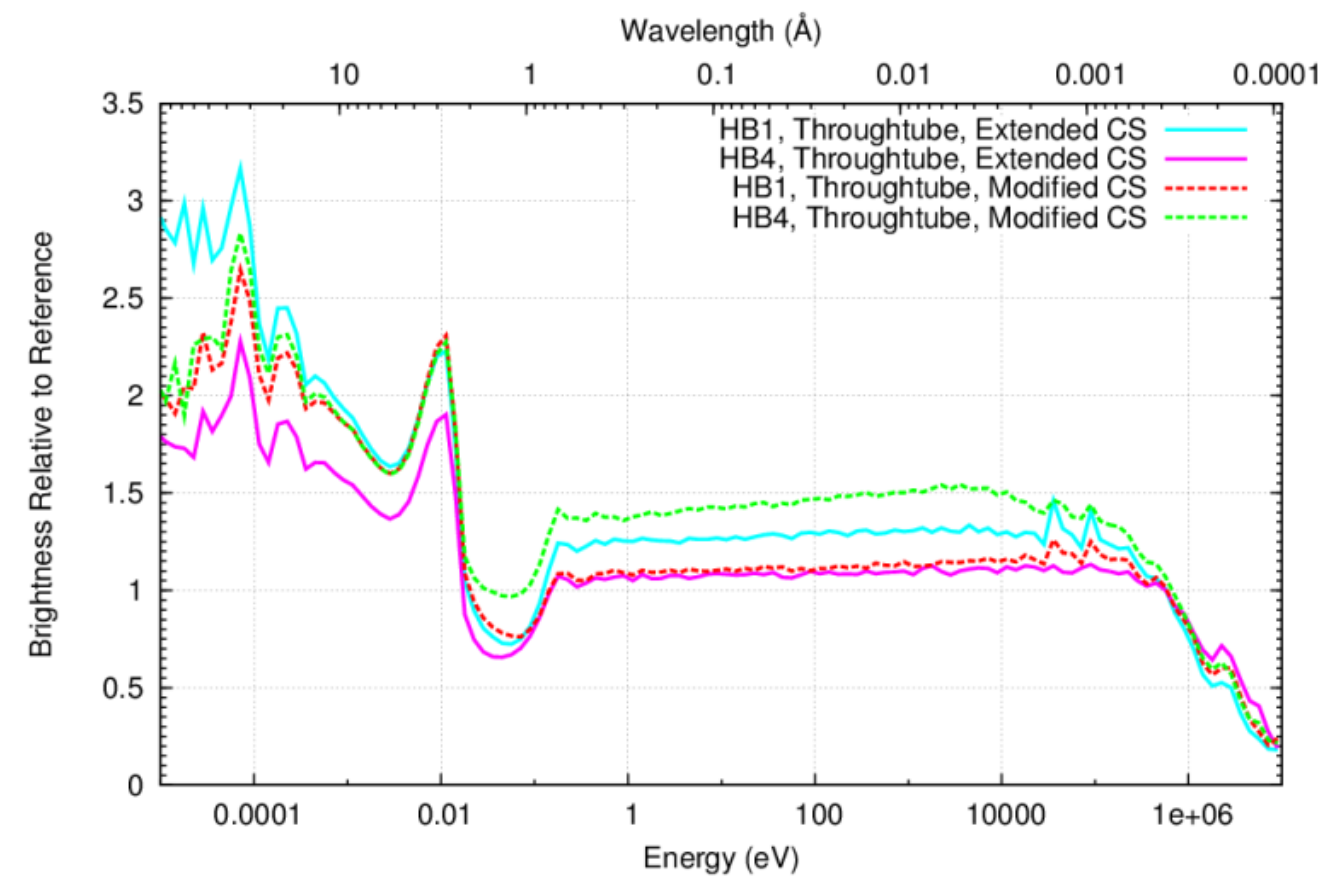

Figure 3.6.7. Cold source brightness versus energy, relative to the reference cold source, for the two CS configurations with the through tube that allow viewing of the CS from HB-4 and HB-1 side.

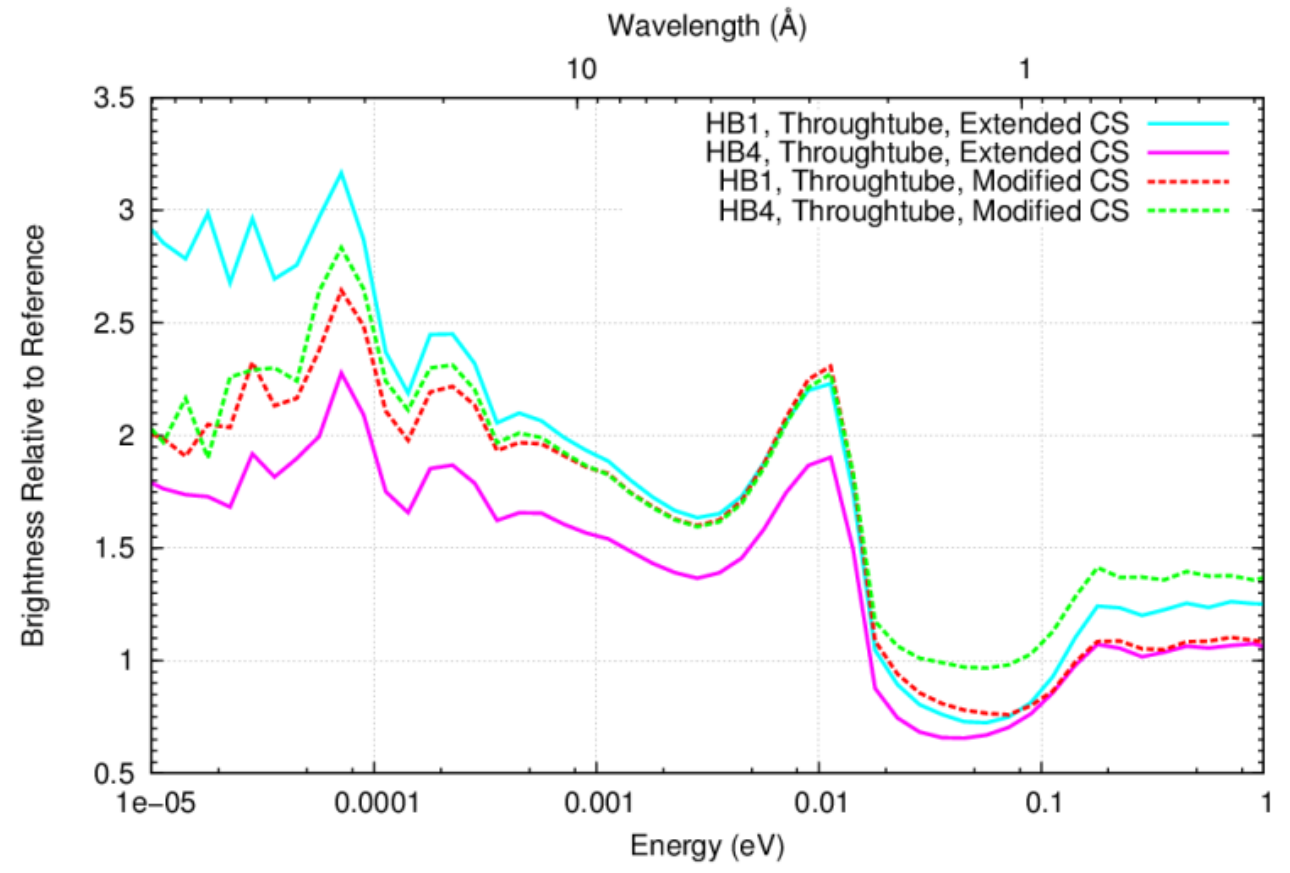

Figure 3.6.8. Cold source brightness versus energy, relative to the reference cold source, for the two CS configurations with the through tube that allow viewing of the CS from HB-4 and HB-1 side, for the energies below $1 \mathrm{eV}$. 


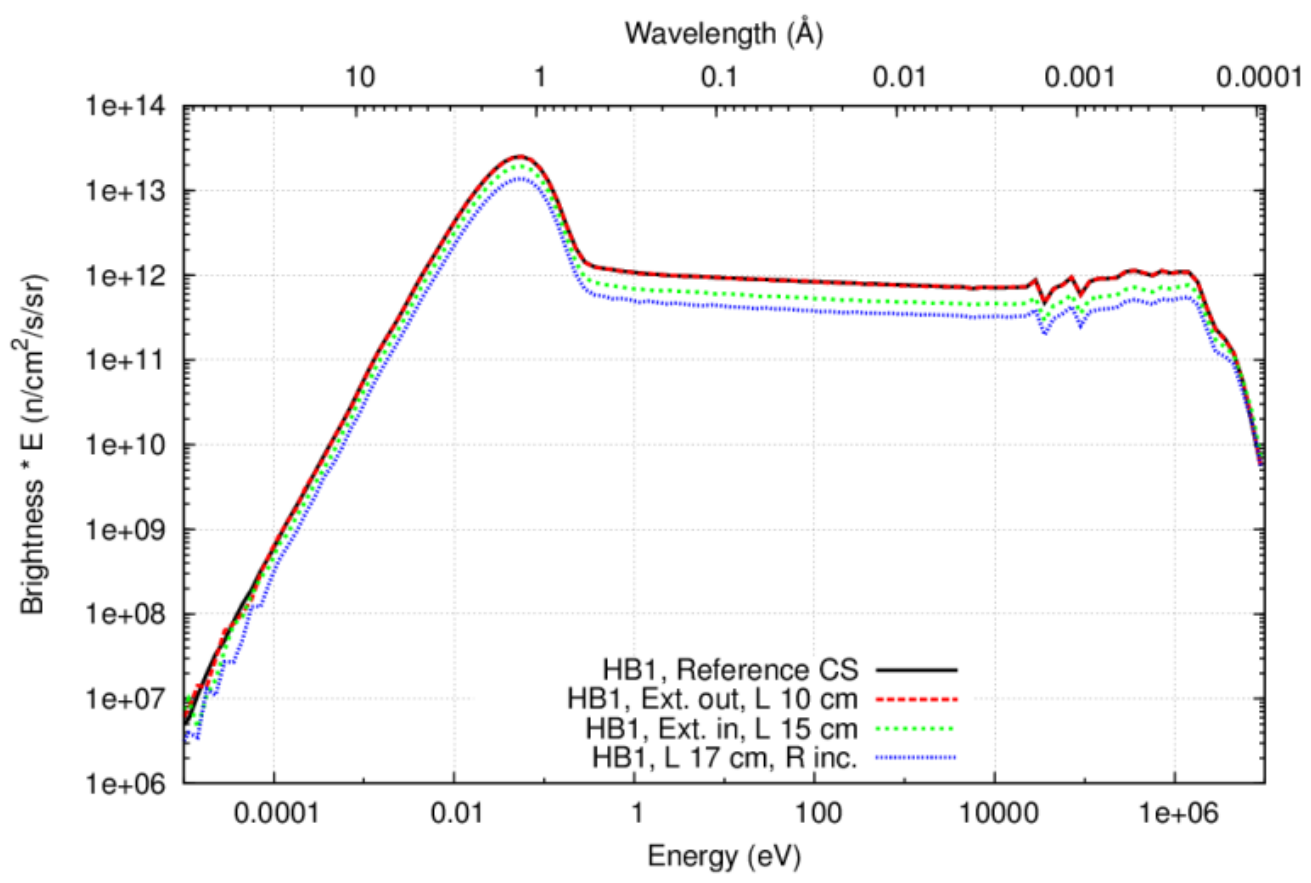

Figure 3.6.9. Brightness of the HB-1 side for the reference CS configuration and for the three CS configurations that keep the HB-4 and HB-1 separated. The three configurations are: CS extended outwards, CS extended inwards and HB-1 retracted, and CS with increased radius, extended inwards, and HB-1 retracted.

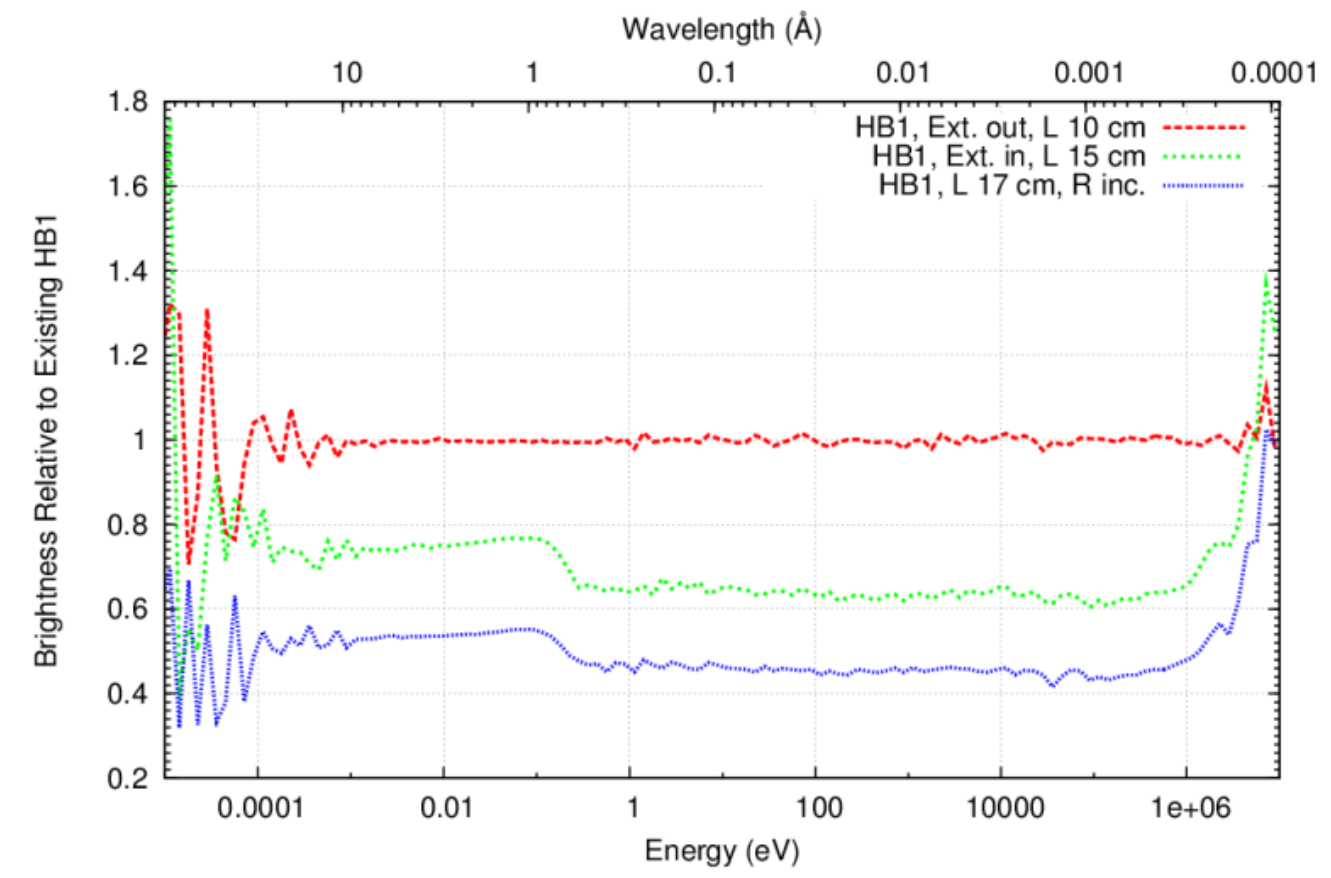

Figure 3.6.10. Brightness of the HB-1 side relative to the HB-1 side brightness of the reference CS configuration, for the three CS configurations that keep the HB-4 and HB-1 separated. 


\subsection{HEATING AND REACTIVITY IMPACT OF CS MODIFICATIONS}

Energy deposited in the CS determines the requirements for the refrigeration system and is an important parameter for the CS design. Besides, the volume of hydrogen in the CS needs to be considered in the accident analysis. For these reasons the volumes of the aluminum and hydrogen, along with the energy deposition in the CS, are given in the Tables 3.7.1 to 3.7.5. Each table is for one of the CS configurations considered. The volumes and the heating are also listed relative to the values for the reference CS. The heating is calculated for the HFIR reactor power of $85 \mathrm{MW}$. Uncertainties associated with the Monte Carlo calculation of the heating are less than $1 \%$ (1-sigma) and are not listed in the tables. For the reference CS, the heating is $2194 \mathrm{~W}$ at the reactor power $85 \mathrm{MW}$ in agreement with the heating of $2144 \mathrm{~W}$ reported in previous analysis [16]. The heating rates in hydrogen for the reference CS are in the range of 1 to $1.3 \mathrm{~W} / \mathrm{cm}^{3}$.

For the CS configurations with significantly increased brightness the heating is around a factor of two higher than for the reference CS (see Tab. 3.6.1 and Tables 3.7.1 - 3.7.5). In all CS configurations analyzed for this report, the CS wall thickness was kept the same as in the present source ( $2 \mathrm{~mm}$ to $2.5 \mathrm{~mm}$ ); however, larger CS volumes may require thicker walls, which will in turn decrease the brightness and increase the heating rate. These effects must be considered in detailed design analyses.

The modified CS configurations caused only very minor changes in effective multiplication factor. For all the CS configurations listed in Table 3.6.1 a small decrease in $k_{\text {eff }}$ was observed. The decrease did not exceed $190 \mathrm{pcm}$. The largest decrease was observed for the configurations with connected HB-1 and HB-4 beamtubes and in configuration with increased CS radius.

Table 3.7.1. Heating of CS of existing design with CS length extended outward*. Hydrogen supply piping was not included in volumes and heating.

\begin{tabular}{|c|c|c|c|c|c|c|c|c|}
\hline \multirow[b]{2}{*}{$\begin{array}{c}\mathbf{L}^{\dagger} \\
(\mathrm{cm})\end{array}$} & \multicolumn{3}{|c|}{ Volume } & \multirow[b]{2}{*}{$\begin{array}{c}\text { Heating } \\
(\mathrm{W})\end{array}$} & \multicolumn{3}{|c|}{ Volume } & \multirow{2}{*}{$\begin{array}{l}\text { Heating } \\
\text { Factor }\end{array}$} \\
\hline & Aluminum & $\begin{array}{l}\text { Hydrogen } \\
\left(\mathrm{cm}^{3}\right)\end{array}$ & Total & & \multicolumn{3}{|c|}{ Relative to Reference CS } & \\
\hline 5 & 162 & 465 & 626 & 2192 & 1.00 & 1.00 & 1.00 & 1.00 \\
\hline 7 & 177 & 602 & 778 & 2384 & 1.09 & 1.30 & 1.24 & 1.09 \\
\hline 9 & 191 & 739 & 931 & 2566 & 1.18 & 1.59 & 1.49 & 1.17 \\
\hline 11 & 206 & 876 & 1083 & 2713 & 1.28 & 1.89 & 1.73 & 1.24 \\
\hline 13 & 221 & 1014 & 1235 & 2862 & 1.37 & 2.18 & 1.97 & 1.30 \\
\hline 15 & 236 & 1151 & 1387 & 2984 & 1.46 & 2.48 & 2.21 & 1.36 \\
\hline 17 & 251 & 1288 & 1539 & 3088 & 1.55 & 2.77 & 2.46 & 1.41 \\
\hline 19 & 266 & 1425 & 1691 & 3179 & 1.65 & 3.07 & 2.70 & 1.45 \\
\hline 21 & 281 & 1563 & 1844 & 3253 & 1.74 & 3.36 & 2.94 & 1.48 \\
\hline 23 & 296 & 1700 & 1996 & 3323 & 1.83 & 3.66 & 3.19 & 1.51 \\
\hline 25 & 311 & 1837 & 2148 & 3374 & 1.92 & 3.95 & 3.43 & 1.54 \\
\hline 26 & 318 & 1906 & 2224 & 3419 & 1.97 & 4.10 & 3.55 & 1.56 \\
\hline
\end{tabular}

*The inner radius of the CS cylindrical body is $4.67 \mathrm{~cm}$ and the outer radius is $4.92 \mathrm{~cm}$.

${ }^{\dagger} \mathrm{L}$ is the length of the CS capsule (reference CS has the length of $5 \mathrm{~cm}$ ).

Volumes and heating are given relative to the reference CS configuration. 
Table 3.7.2. Heating of existing CS with CS length extended inward*.

Hydrogen supply piping was not included in volumes and heating.

\begin{tabular}{|c|c|c|c|c|c|c|c|c|}
\hline \multirow[b]{2}{*}{$\begin{array}{c}\mathbf{L}^{\dagger} \\
(\mathrm{cm})\end{array}$} & \multicolumn{3}{|c|}{ Volume } & \multirow[b]{2}{*}{$\begin{array}{l}\text { Heating } \\
\text { (W) }\end{array}$} & \multicolumn{3}{|c|}{ Volume } & \multirow{2}{*}{$\begin{array}{l}\text { Heating } \\
\text { Factor }\end{array}$} \\
\hline & Aluminum & $\begin{array}{l}\text { Hydrogen } \\
\left(\mathrm{cm}^{3}\right)\end{array}$ & Total & & $\begin{array}{r}\text { Aluminum } \\
\mathrm{R}\end{array}$ & $\begin{array}{l}\text { Hydrogen } \\
\text { lative to Ref }\end{array}$ & $\begin{array}{l}\text { Total } \\
\text { ence CS }\end{array}$ & \\
\hline 5 & 162 & 465 & 626 & 2194 & 1.00 & 1.00 & 1.00 & 1.00 \\
\hline 7 & 177 & 602 & 778 & 2557 & 1.09 & 1.30 & 1.24 & 1.17 \\
\hline 9 & 191 & 739 & 931 & 2944 & 1.18 & 1.59 & 1.49 & 1.34 \\
\hline 11 & 206 & 876 & 1083 & 3340 & 1.28 & 1.89 & 1.73 & 1.52 \\
\hline 13 & 221 & 1014 & 1235 & 3742 & 1.37 & 2.18 & 1.97 & 1.71 \\
\hline 15 & 236 & 1151 & 1387 & 4133 & 1.46 & 2.48 & 2.21 & 1.88 \\
\hline 17 & 251 & 1288 & 1539 & 4525 & 1.55 & 2.77 & 2.46 & 2.06 \\
\hline 19 & 266 & 1425 & 1691 & 4903 & 1.65 & 3.07 & 2.70 & 2.23 \\
\hline 21 & 281 & 1563 & 1844 & 5281 & 1.74 & 3.36 & 2.94 & 2.41 \\
\hline 23 & 296 & 1700 & 1996 & 5635 & 1.83 & 3.66 & 3.19 & 2.57 \\
\hline 25 & 311 & 1837 & 2148 & 5955 & 1.92 & 3.95 & 3.43 & 2.71 \\
\hline 26 & 318 & 1906 & 2224 & 6113 & 1.97 & 4.10 & 3.55 & 2.79 \\
\hline
\end{tabular}

*The inner radius of the CS cylindrical body is $4.67 \mathrm{~cm}$ and the outer radius is $4.92 \mathrm{~cm}$.

${ }^{\dagger} \mathrm{L}$ is the length of the CS capsule (reference CS has the length of $5 \mathrm{~cm}$ ).

Volumes and heating are given relative to the reference CS configuration. 
Table 3.7.3. Heating of CS with increased radius* and with CS length extended inward. Hydrogen supply piping was not included in volumes and heating.

\begin{tabular}{|c|c|c|c|c|c|c|c|c|}
\hline \multirow[b]{2}{*}{$\begin{array}{c}\mathbf{L}^{\dagger} \\
(\mathrm{cm}) \\
\end{array}$} & \multicolumn{3}{|c|}{ Volume } & \multirow[b]{2}{*}{$\begin{array}{c}\text { Heating } \\
(\mathrm{W})\end{array}$} & \multicolumn{3}{|c|}{ Volume } & \multirow{2}{*}{$\begin{array}{c}\text { Heating } \\
\text { Factor }\end{array}$} \\
\hline & Aluminum & $\begin{array}{l}\text { Hydrogen } \\
\left(\mathrm{cm}^{3}\right)\end{array}$ & Total & & \multirow{2}{*}{\multicolumn{3}{|c|}{ Relative to Reference CS }} & \\
\hline 5 & 190 & 621 & 810 & 2675 & 1.17 & & & 1.22 \\
\hline 7 & 208 & 829 & 1037 & 3186 & 1.29 & 1.78 & 1.66 & 1.45 \\
\hline 9 & 226 & 1037 & 1264 & 3723 & 1.40 & 2.23 & 2.02 & 1.70 \\
\hline 11 & 245 & 1246 & 1491 & 4272 & 1.51 & 2.68 & 2.38 & 1.95 \\
\hline 13 & 263 & 1454 & 1717 & 4838 & 1.63 & 3.13 & 2.74 & 2.20 \\
\hline 15 & 281 & 1663 & 1944 & 5385 & 1.74 & 3.58 & 3.10 & 2.45 \\
\hline 17 & 300 & 1871 & 2171 & 5944 & 1.85 & 4.03 & 3.47 & 2.71 \\
\hline 19 & 318 & 2079 & 2397 & 6460 & 1.97 & 4.47 & 3.83 & 2.94 \\
\hline 21 & 336 & 2288 & 2624 & 6974 & 2.08 & 4.92 & 4.19 & 3.18 \\
\hline 23 & 355 & 2496 & 2851 & 7472 & 2.19 & 5.37 & 4.55 & 3.41 \\
\hline 25 & 373 & 2705 & 3078 & 7937 & 2.31 & 5.82 & 4.91 & 3.62 \\
\hline 26 & 391 & 2913 & 3304 & 8361 & 2.42 & 6.27 & 5.28 & 3.81 \\
\hline
\end{tabular}

*The inner radius increased by $1.09 \mathrm{~cm}$, to inner radius of $5.76 \mathrm{~cm}$ and outer radius of $6.01 \mathrm{~cm}$. ${ }^{\dagger} \mathrm{L}$ is the length of the CS capsule (reference CS has the length of $5 \mathrm{~cm}$ ).

Volumes and heating are given relative to the reference CS configuration. 
Table 3.7.4. Heating of existing CS designs of various lengths placed in through tube* and centered around reactor core. Hydrogen supply piping was not included in volumes and heating.

\begin{tabular}{|c|c|c|c|c|c|c|c|c|}
\hline \multirow[b]{2}{*}{$\begin{array}{c}\mathbf{L}^{\dagger} \\
(\mathrm{cm}) \\
\end{array}$} & \multicolumn{3}{|c|}{ Volume } & \multirow[b]{2}{*}{$\begin{array}{c}\text { Heating } \\
(\mathrm{W})\end{array}$} & \multicolumn{3}{|c|}{ Volume } & \multirow{2}{*}{$\begin{array}{l}\text { Heating } \\
\text { Factor }\end{array}$} \\
\hline & Aluminum & $\begin{array}{l}\text { Hydrogen } \\
\left(\mathrm{cm}^{3}\right)\end{array}$ & Total & & \multicolumn{3}{|c|}{ Relative to Reference CS } & \\
\hline 5 & 162 & 465 & 626 & 2739 & 1.00 & 1.00 & 1.00 & 1.25 \\
\hline 7 & 177 & 602 & 778 & 3076 & 1.09 & 1.30 & 1.24 & 1.40 \\
\hline 9 & 191 & 739 & 931 & 3408 & 1.18 & 1.59 & 1.49 & 1.55 \\
\hline 11 & 206 & 876 & 1083 & 3729 & 1.28 & 1.89 & 1.73 & 1.70 \\
\hline 13 & 221 & 1014 & 1235 & 4060 & 1.37 & 2.18 & 1.97 & 1.85 \\
\hline 15 & 236 & 1151 & 1387 & 4359 & 1.46 & 2.48 & 2.21 & 1.99 \\
\hline 17 & 251 & 1288 & 1539 & 4668 & 1.55 & 2.77 & 2.46 & 2.13 \\
\hline 19 & 266 & 1425 & 1691 & 4966 & 1.65 & 3.07 & 2.70 & 2.26 \\
\hline 21 & 281 & 1563 & 1844 & 5241 & 1.74 & 3.36 & 2.94 & 2.39 \\
\hline 23 & 296 & 1700 & 1996 & 5521 & 1.83 & 3.66 & 3.19 & 2.52 \\
\hline 24 & 303 & 1768 & 2072 & 5649 & 1.88 & 3.81 & 3.31 & 2.57 \\
\hline
\end{tabular}

*The inner radius of the CS cylindrical body is $4.67 \mathrm{~cm}$ and the outer radius is $4.92 \mathrm{~cm}$.

${ }^{\dagger} \mathrm{L}$ is the length of the CS capsule (reference CS has the length of $5 \mathrm{~cm}$ ).

Volume and heating is given relative to the reference CS configuration. 
Table 3.7.5. Heating of modified CS designs of various lengths placed in through tube* and centered around reactor core. Hydrogen supply piping was not included in volumes and heating.

\begin{tabular}{|c|c|c|c|c|c|c|c|c|c|}
\hline \multirow[b]{2}{*}{$\begin{array}{c}\mathbf{T}^{\dagger} \\
(\mathrm{cm}) \\
\end{array}$} & \multirow[b]{2}{*}{$\begin{array}{c}\text { Theta } \\
\text { (degrees) }\end{array}$} & \multicolumn{3}{|c|}{ Volume } & \multirow[b]{2}{*}{$\begin{array}{c}\text { Heating } \\
(\mathrm{W})\end{array}$} & \multicolumn{3}{|c|}{ Volume } & \multirow{2}{*}{$\begin{array}{l}\text { Heating } \\
\text { Factor }\end{array}$} \\
\hline & & Aluminum & $\begin{array}{l}\text { Hydrogen } \\
\left(\mathrm{cm}^{3}\right)\end{array}$ & Total & & \multicolumn{3}{|c|}{ Relative to Reference $\mathrm{CS}^{\S}$} & \\
\hline 6 & 0 & 75 & 412 & 487 & 1829 & 0.46 & 0.89 & 0.78 & 0.83 \\
\hline 6 & 10 & 76 & 419 & 494 & 1858 & 0.47 & 0.90 & 0.79 & 0.85 \\
\hline 6 & 20 & 80 & 439 & 518 & 1932 & 0.49 & 0.94 & 0.83 & 0.88 \\
\hline 6 & 30 & 86 & 476 & 562 & 2062 & 0.53 & 1.02 & 0.90 & 0.94 \\
\hline 6 & 40 & 98 & 538 & 636 & 2290 & 0.60 & 1.16 & 1.01 & 1.04 \\
\hline 6 & 50 & 116 & 641 & 758 & 2647 & 0.72 & 1.38 & 1.21 & 1.21 \\
\hline 6 & 60 & 149 & 824 & 974 & 3262 & 0.92 & 1.77 & 1.55 & 1.49 \\
\hline 6 & 65 & 177 & 975 & 1152 & 3718 & 1.09 & 2.10 & 1.84 & 1.69 \\
\hline 6 & 70 & 218 & 1205 & 1424 & 4370 & 1.35 & 2.59 & 2.27 & 1.99 \\
\hline 6 & 75 & 289 & 1593 & 1881 & 5296 & 1.79 & 3.43 & 3.00 & 2.41 \\
\hline 5 & 75 & 260 & 1328 & 1587 & 4778 & 1.61 & 2.86 & 2.53 & 2.18 \\
\hline 7 & 70 & 240 & 1406 & 1646 & 4790 & 1.49 & 3.03 & 2.63 & 2.18 \\
\hline 8 & 65 & 212 & 1300 & 1512 & 4449 & 1.31 & 2.80 & 2.41 & 2.03 \\
\hline 9 & 65 & 230 & 1463 & 1692 & 4803 & 1.42 & 3.15 & 2.70 & 2.19 \\
\hline
\end{tabular}

*The inner radius of the CS cylindrical body is $4.67 \mathrm{~cm}$ and the outer radius is $4.92 \mathrm{~cm}$.

${ }^{\dagger} \mathrm{T}$ is the thickness of the CS.

Theta is the angle of the inclination (see Section 3.5).

${ }^{\S}$ olume and heating is given relative to the reference CS configuration. 


\section{COMPARISON WITH EXPERIMENTS}

The analyses presented in this report were performed with computer codes (primarily MCNPX version 2.7.0 [10]) and data that were routinely used for the design calculations for the SNS first target station, and the preliminary design of the SNS second target station, and the validity and reliability of the simulations is well established. The HFIR and CS model prepared for the previous analysis [3] was used as a starting point which for the CS modifications analyzed.

No dedicated experiments were performed to validate the simulations presented in this report. However, the HFIR CS brightness was measured by Robertson and Iverson in an experiment performed in September 2007 [17] and will be compared with simulations in section 4.1. The effect of para-hydrogen fraction on the neutronic performance of liquid hydrogen moderator was studied experimentally by Ooi and co-workers in Japan in 2010 [15] and will be compared with the simulations in section 4.2 .

\subsection{COMPARISON WITH MEASURED HFIR CS BRIGHTNESS}

Robertson and Iverson used an apparatus which consisted of a disk chopper, a neutron detector, and a data acquisition system to record the number of neutrons arriving at the detector as a function of the neutron time-of-flight (TOF). The disk chopper was located immediately downstream of the external collimator in the beamline HB-4, delivering the neutron beam from the cold source. The neutron detector was a Reuter Stokes parallel plate fission counter with the anode located 2,473mm from the center (thickness) of the chopper disk. The TOF spectrum was collected as a histogram with $10 \mu$ s time channels. TOF spectrum was then converted into CS brightness versus energy curve as described in [17]. It was pointed out that a major source of uncertainty of the measured CS brightness was the approximate treatment of the rectangular and circular apertures of the collimation system between the source and the detector in the calculation of the detector acceptance. However, the uncertainty estimates for the measured CD brightness were not provided.

The measured CS brightness versus energy is shown in Fig. 4.1.1, together with the calculated brightness for the CS with 34\% para-hydrogen and $99 \%$ para-hydrogen. The measured brightness is for the reactor power level of $85 \mathrm{MW}$ and moderator temperature of $22.5 \mathrm{~K}$. The calculated values are also normalized to the reactor power of $85 \mathrm{MW}$. Fig. 4.1.2 shows the same three curves as Fig. 4.1.1, however the calculated brightness curves are normalized to the measured brightness value at the $0.113 \mathrm{eV}$, to allow easier comparison of the brightness versus energy variations.

The calculations agree with the measurements to within $\sim 40 \%$ to $50 \%$. The measured brightnesses are consistently higher that the calculated values over the full energy range considered. The shape of the measured brightness versus energy curve agree remarkably well with the calculated shape for the CS with 35\% para-hydrogen, while it differs considerably from the shape for the $99 \%$ para-hydrogen, therefore reinforcing the assessment that the HFIR CS likely operates at $\sim 35 \%$ para-hydrogen fraction. Overall the simulations and the measurements agree reasonably well; however, the root cause for the discrepancies is not clear and may lay with the simulations, the measurements, or is split between the two. 


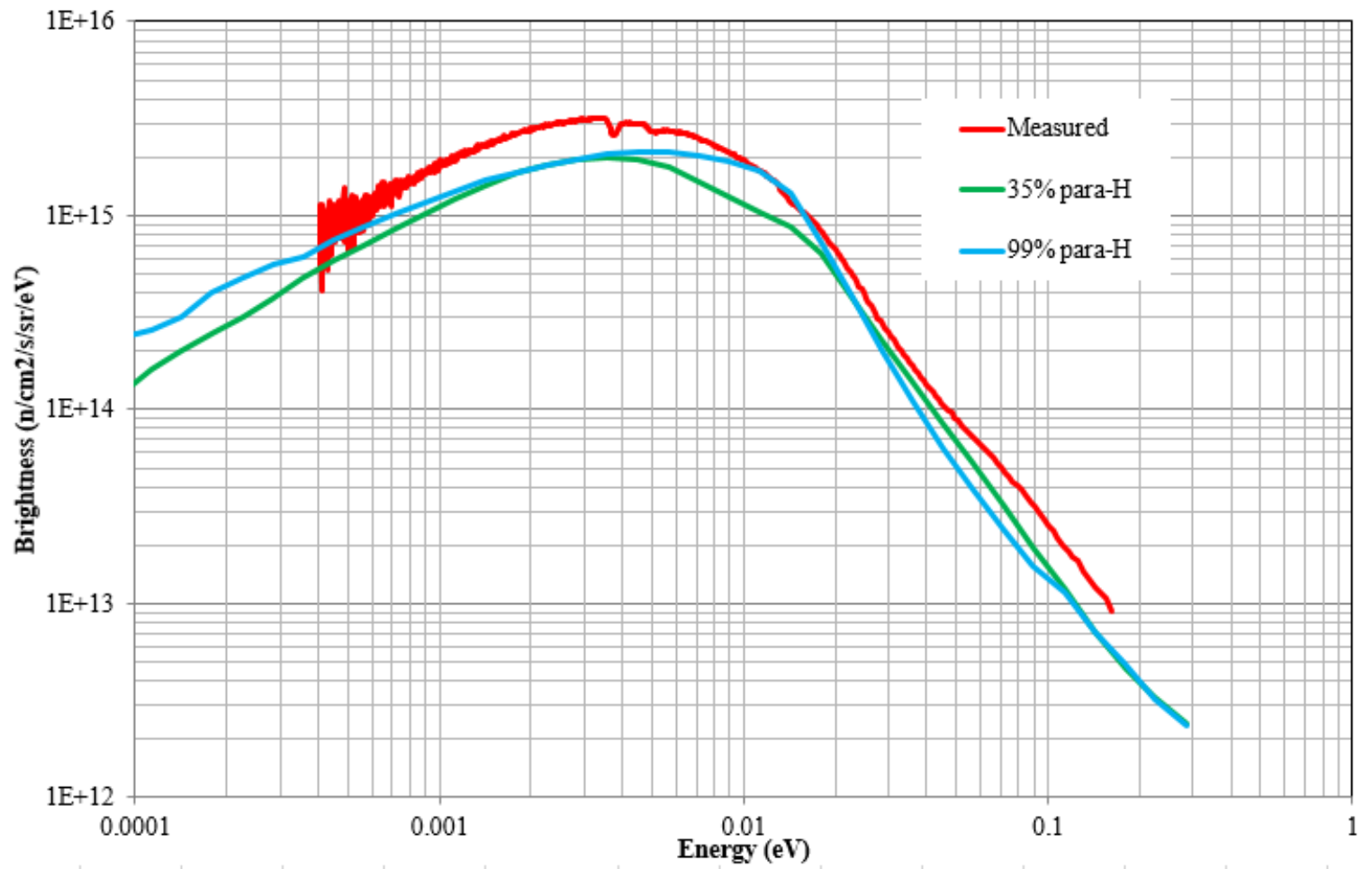

Figure 4.1.1 Measured and calculated CS brightness versus energy. The calculated brightness is given for the $35 \%$ and $99 \%$ para-hydrogen fraction.

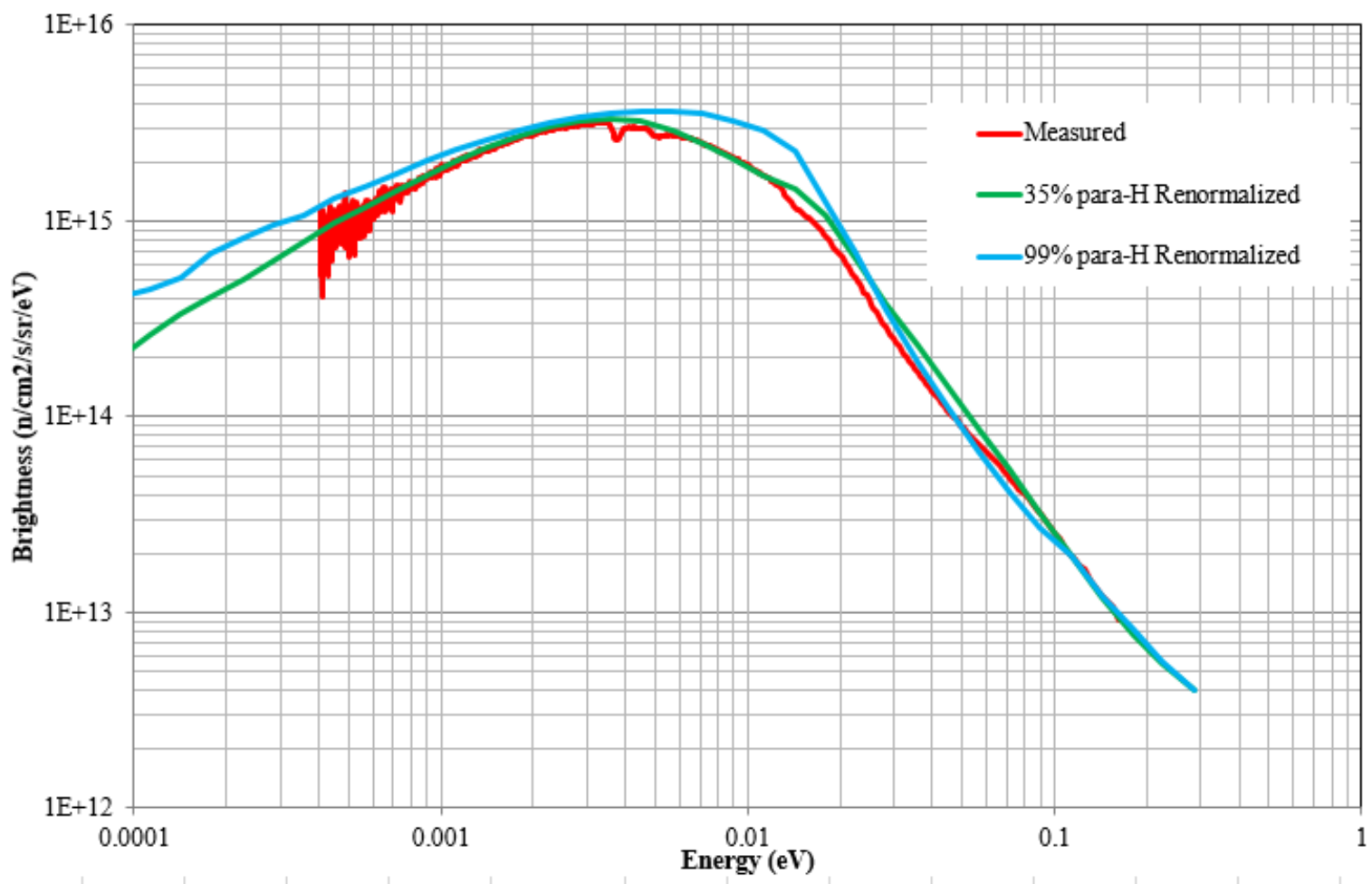

Figure 4.1.2 Comparison of the measured and calculated CS brightness versus energy curves. The calculated brightnesses are normalized to the measured brightness at $\sim 0.113 \mathrm{eV}$. The calculated brightness is given for the $35 \%$ and $99 \%$ para-hydrogen fraction. 


\subsection{COMPARISON WITH MEASURED EFFECT OF PARA-HYDROGEN FRACTION}

In 2010 Ooi and coworkers studied experimentally the effect of ortho/para hydrogen ratio on the neutronic performance of a liquid hydrogen moderator for a pulsed neutron source [15]. The experiment was performed at the Hokkaido University. Their experimental apparatus is shown in Fig. 4.2.1 A 45-meV electron linac delivered 40ns electron pulses to the lead target. Neutron were produced in the lead via $(\gamma . n)$ reaction. Rectangular moderator $(5 \mathrm{~cm} \times 12 \mathrm{~cm} \times 12 \mathrm{~cm})$ was placed above the target. The volume of the moderator was $720 \mathrm{~cm}^{3}$, similar to the HFIR CS volume of $465 \mathrm{~cm}^{3}$, and the thickness in the viewed direction was $5 \mathrm{~cm}$ measurements, which is equal to the length of the HFIR CS capsule. ${ }^{3} \mathrm{He}$ detectors were used to measure the neutron energy spectra and the emission them distributions (pulse shapes). The time-of-flight method was used to determine neutron energy spectra. To accelerate the ortho-to-para-hydrogen conversion a catalyst was used $\left(\mathrm{Fe}(\mathrm{OH})_{3}\right)$. To change the para-hydrogen fraction was the temperature of the catalyst was changed. The para-hydrogen fraction was measured online when filling the moderator and was confirmed by Raman spectroscopy after the experiment. The moderator was kept at the temperature of $18^{\circ} \mathrm{K}$.

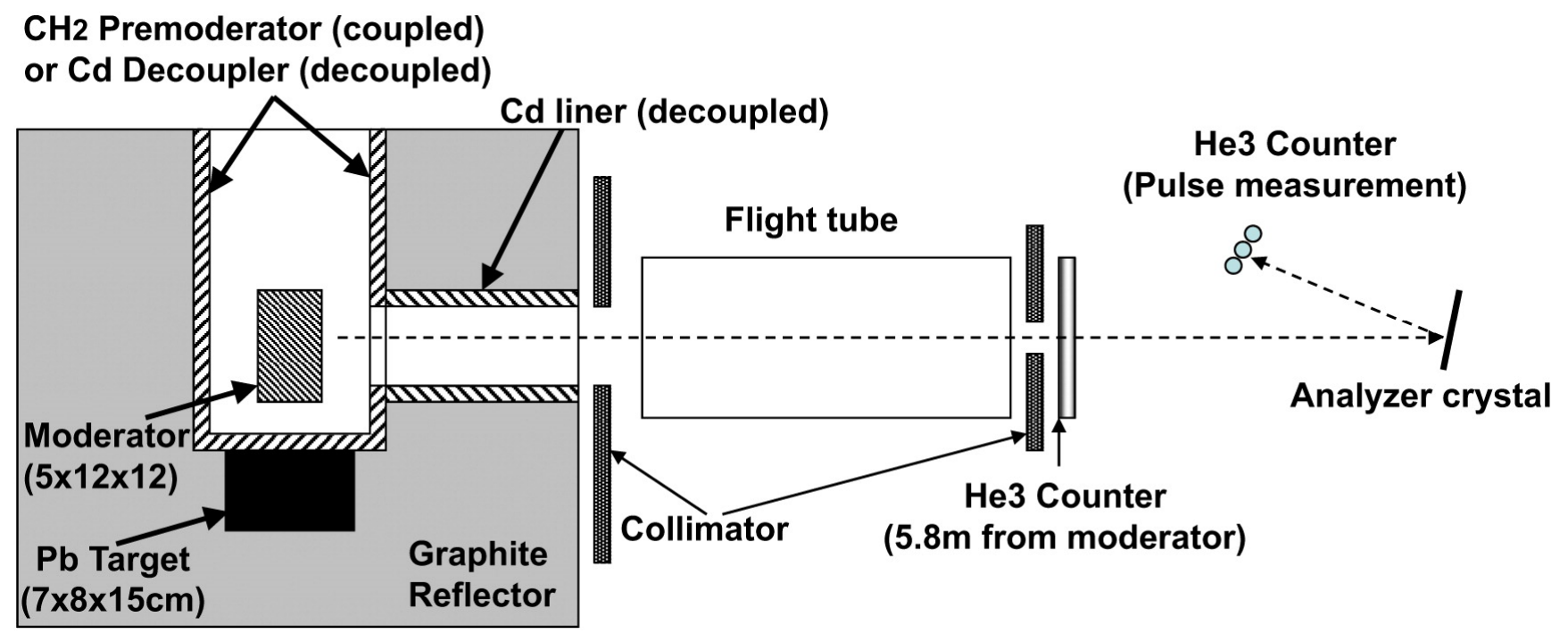

Figure 4.2.1 Experimental apparatus used by Ooi and co-workers. ${ }^{1}$

Neutron spectra were measured for para-hydrogen fractions of 35\%,60\%, 90\%, 95\% and 99\%. Both coupled and decoupled moderators were measured. The measured spectra are shown in Fig. 4.2.2. More informative than the spectra are the ratios of the spectra shown in the bottom part of the Fig.4.2.2, that clearly point out the spectral changes due to the change in para-hydrogen fraction. Moderator brightness is proportional to the measured intensity, therefore the ratios of the intensities for the selected two para-hydrogen fractions are the same as the ratios of brightnesses. The relevant data for comparison are those obtained for the coupled hydrogen moderator.

\footnotetext{
${ }^{1}$ Reprinted from NIMA, Vol 659, Motoki Ooi et al., Experimental studies of the effect of the ortho/para ratio on the neutronic performance of a liquid hydrogen moderator for a pulsed neutron source, $\mathrm{p}$ 62, Copyright (2011), with permission from Elsevier.
} 
Fig. 4.2.3 Presents the measured (at the right side) and simulated moderator brightness relative to the brightness of the moderator filled with 35\% para-hydrogen. The measured values, shown on the right side, are from the experiment by Ooi for the coupled moderator (bottom left part from the Fig. 4.2.2). The calculated values, shown on the right side, are from the current work and for the existing HFIR CS. Excellent agreement in the shapes of the measured and calculated curves clearly indicates that the effect of changing the para-hydrogen fraction, predicted with simulations, is consistent with the effect observed with the measurements.

As noted in section 3.6, the shapes of the curves of the brightness increase versus energy are remarkably similar for all considered CS configurations and are caused by change from $35 \%$ para-hydrogen to $99 \%$ para-hydrogen. The highest increase is typically observed at $\sim 10 \mathrm{meV}$ and $\sim 0.1 \mathrm{meV}$. Between these two peaks the brightness may decrease (as we see here for the CS thickness $\sim 5 \mathrm{~cm}$ ) or may show smaller increase (see Fig. 3.6.8). Towards higher energies, between $20 \mathrm{meV}$ and $200 \mathrm{meV}$ brightness typically decreases. Finally, changing para-hydrogen fraction has no effect on brightness at neutron energies higher than $200 \mathrm{meV}$
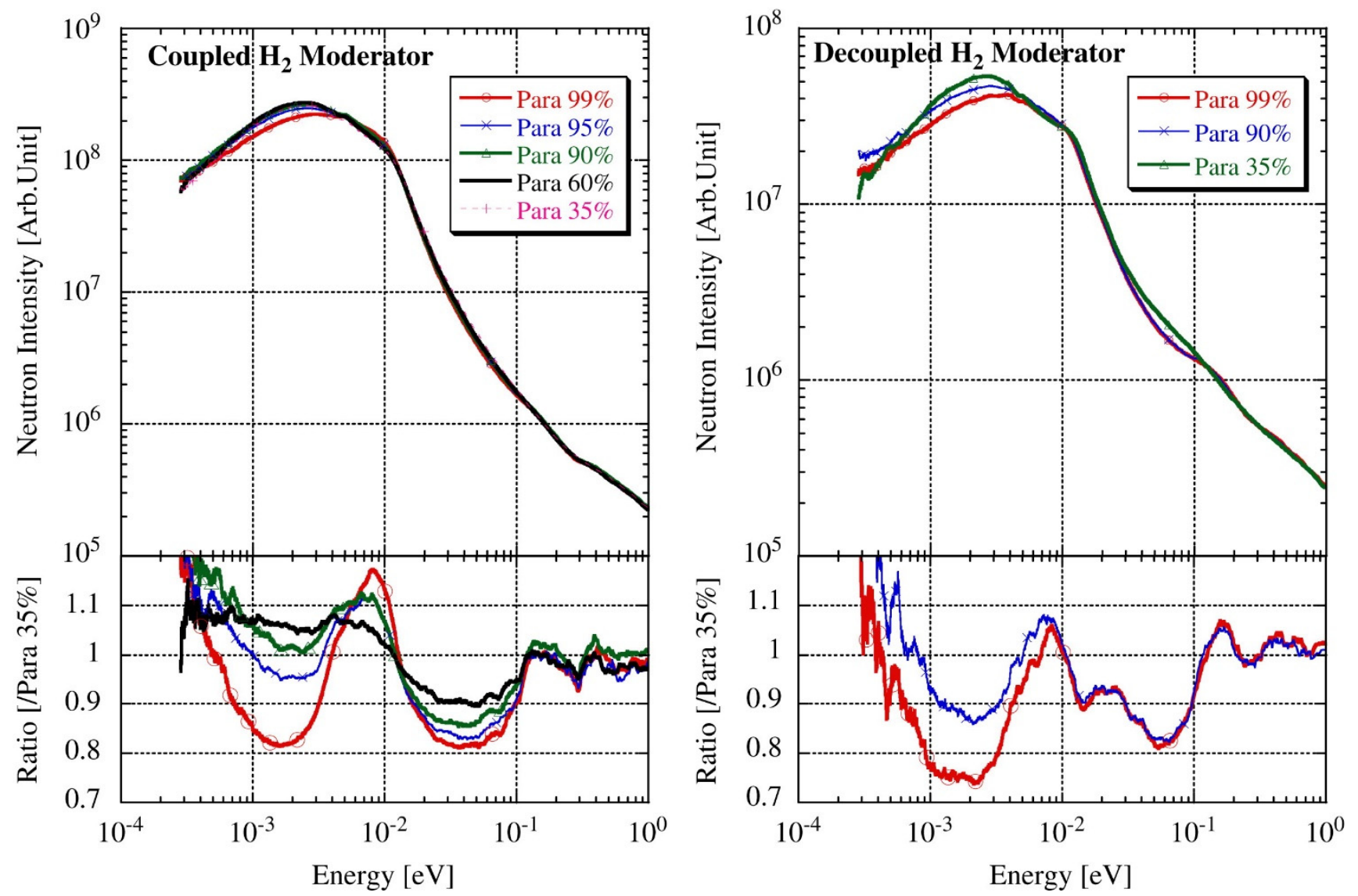

Figure 4.2.2 Neutron energy spectra and intensity ratios for the coupled moderator (left) and for the decoupled moderator (right). Intensity ratios are normalized by para-hydrogen $35 \%$ data. ${ }^{2}$

\footnotetext{
${ }^{2}$ Reprinted from NIMA, Vol 659, Motoki Ooi et al., Experimental studies of the effect of the ortho/para ratio on the neutronic performance of a liquid hydrogen moderator for a pulsed neutron source, $\mathrm{p}$ 62, Copyright (2011), with permission from Elsevier.
} 


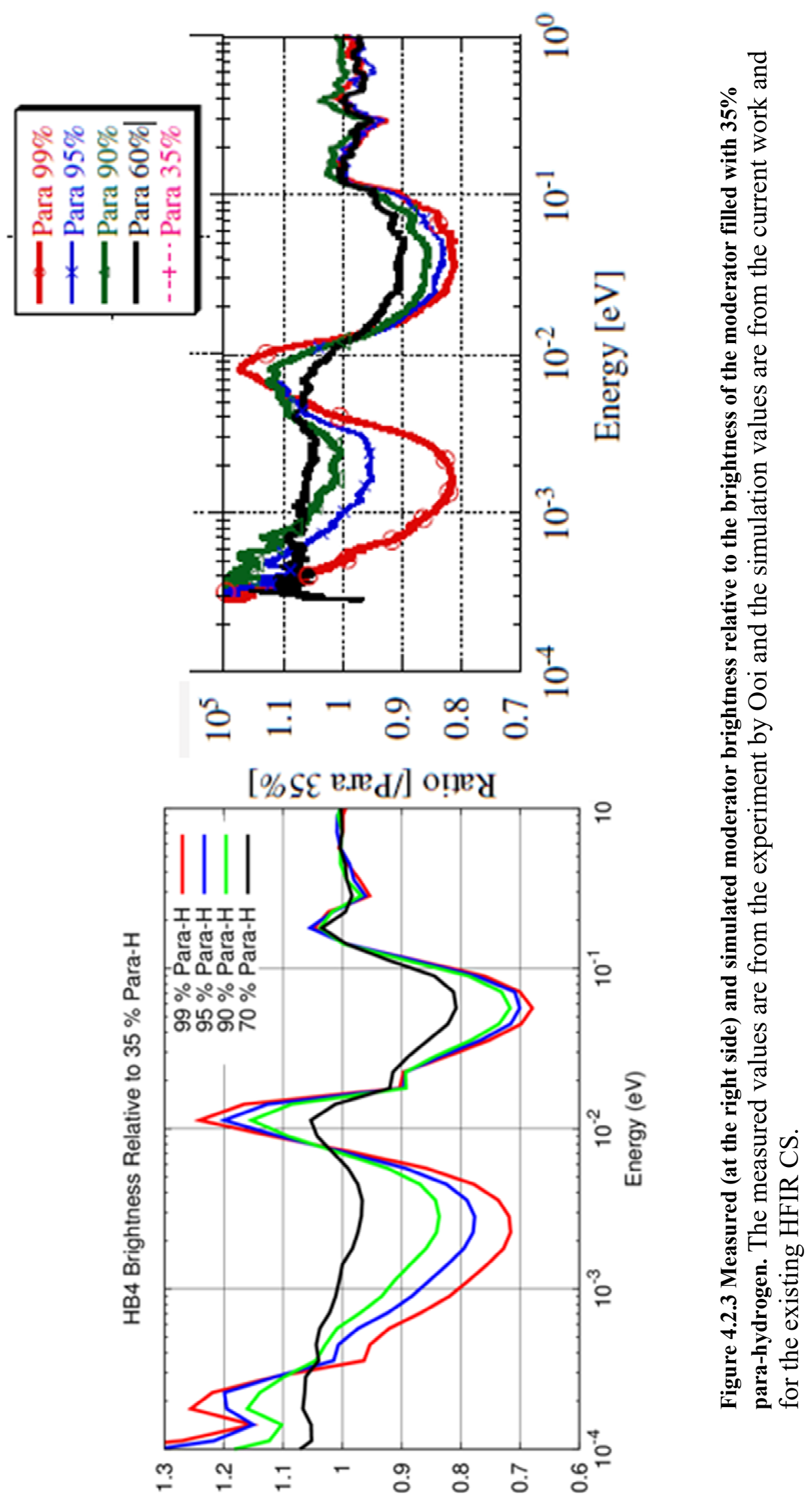

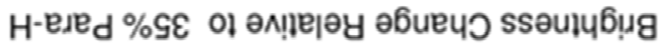




\section{SUMMARY}

Several cold source configurations that provide $40 \%$ to $90 \%$ higher brightness than the reference cold source in broad ranges of wavelengths longer than $\sim 2 \AA$ were identified. These CS configurations use hydrogen volumes 2.5 to 4 times larger than the reference source, require a nearly pure para-hydrogen for operation ( $99 \%$ para-hydrogen was assumed in the calculations), and impose heating 1.9 to 2.7 times higher than the reference cold source. Implementation would require adding an ortho-para hydrogen converter and increasing the refrigeration capacity. The brightness increase varies considerably with energy through the cold and thermal neutron range; however, the shapes of the curves of the brightness-increase-versus-energy are very similar for all the configurations analyzed. Up to 2.3 times higher brightness is observed around $3 \AA$ wavelength. The even higher gains of 3.1 at $35 \AA$ are practically of lower interest. Meanwhile, around $1.5 \AA$ a brightness decrease of up to $50 \%$ is seen for all but one configuration. The configurations with connected HB-1 and HB-4 beamtubes allow the extraction of neutron beams from both sides of the cold source, therefore effectively doubling the potential number of instruments served, while providing brightness increases in the $40 \%$ to $90 \%$ range. A higher intensity neutron source, which would deliver $\sim 2$ times more neutrons down the beamtube, at brightness increased up to $45 \%$, can be obtained by increasing the radius of the HB- 4 and the cold source by $\sim 1 \mathrm{~cm}$; which can be realized within the restrictions imposed by the reactor vessel flanges. Comparison to measured spectral data by Ooi et al. confirm the spectral changes due to ortho-para variations and the magnitude of the effects. 


\section{REFERENCES}

1. R. D. Cheverton and T. M. Sims, HFIR Core Nuclear Design, ORNL-4621 (July, 1971).

2. F. X. Gallmeier and I. Remec, Evaluation of HFIR Thermal Neutron Beam Upgrade Options, SNS-106100200-TR0235-R00, Oak Ridge National Laboratory, Oak Ridge, Tennessee (November 2017).

3. J. A. Bucholz, "Physics Analyses in the Design of the HFIR Cold Neutron Source," pp 29-40, BgNS Transactions, Vol 5, No. 1, July 2000, which was a special issue dedicated to the "XIII International School on Nuclear Physics, Neutron Physics, and Nuclear Energy" held in Varna (Bulgaria), Sept 27-Oct 3, 1999.

4. E. B. Iverson and J. M. Carpenter, Kinetics of irradiated liquid hydrogen, ICANS-XVI, Proceedings of the International Collaboration on Advanced Neutron Sources, Neuss, Germany, pp. 707-718 (2003).

5. R. E. Williams et al., The liquid hydrogen moderator at the NIST research reactor, Proceedings of the International Workshop on Cold Moderators for Pulsed Neutron Sources, pp. 79-86, (1997).

6. T. Kai et al., Coupled hydrogen moderator optimization with ortho/para hydrogen ratio, Nucl. Inst. Meth. A, 523, pp 398-414 (2004).

7. K. Batkov et al., Unperturbed moderator brightness in pulsed neutron sources, Nucl. Inst. Meth. A, 729, pp. 500-505, (2013).

8. Conceptual Design Report Proton Power Upgrade Project, ORNL/TM-12016/672, Oak Ridge National Laboratory, Oak Ridge, Tennessee (August 2017).

9. F. X. Gallmeier et al., Conceptual moderator studies for the Spallation Neutron Source shortpulse second target station, Rev Sci. Inst. 87, 063304 (2016).

10. MCNPX User's Manual version 2.7.0, The MCNPX Team, LA-CP-11-00438, Los Alamos National Laboratory, April 2011.

11. D. E. Peplow, A Computational Model of the High Flux Isotope Reactor for the Calculation of Cold Source, Beam Tube, and Guide Hall Nuclear Parameters, ORNL/TM-2004/251, Oak Ridge National Laboratory, Oak Ridge, Tennessee, November 2004.

12. N. Xoubi and R. T. Primm III, Modeling of the High Flux Isotope Reactor Cycle 400, ORNL/TM-2004/251, Oak Ridge National Laboratory, Oak Ridge, Tennessee, August 2005.

13. F. B. Brown, J. E. Sweezy, R. B. Hayes, "Monte Carlo Parameter Studies and Uncertainty Analyses with MCNP5", LA-UR-04-0499, Los Alamos National Laboratory, Los Alamos, April 2004.

14. S. W. Mosher et al., ADVANTG-An Automated Variance Reduction Parameter Generator, ORNL/TM-2013/416 Rev. 1, Oak Ridge National Laboratory, Oak Ridge (August 2015).

15. Motoki Ooi et al., Experimental studies of the effect of the ortho/para ratio on the neutronic performance of a liquid hydrogen moderator for a pulsed neutron source, Nucl. Inst. Meth. A, 659, pp. 61-68 (2011).

16. J. A. Bucholz, private communication to I. Remec, Oct. $18^{\text {th }}, 2012$.

17. J. L. Robertson and E. B. Iverson, Measurement of the Neutron Spectrum of the HB-4 Cold Source at The High Flux Isotope Reactor at Oak Ridge National Laboratory, Reactor Dosimetry State of the Art 2008, Proc. Of the $13^{\text {th }}$ International Symposium, ISBM-13 978981-4271-10-3, pp.85-93 (2008). 